Sulfamidation of 2-arylaldehydes and ketones with chloramine-T

Thomas Baumann, Michael Bächle, Stefan Bräse*

Supplementary Information 
Analytic

NMR:

$\begin{array}{lll}\text { Bruker AC } 250 & { }^{1} \mathrm{H}: 250 \mathrm{MHz} & { }^{13} \mathrm{C}: 62.5 \mathrm{MHz} \\ \text { Bruker AM 400 } & 400 \mathrm{MHz} & 100 \mathrm{MHz}\end{array}$

Al shifts $\delta$ were assigned in parts per million (ppm), while the solvent signal was used as reference. $\left(\mathrm{CHCl}_{3}\right.$ of $\left[\mathrm{D}_{1}\right]$-chloroform $\left(7.26 \mathrm{ppm} \text { for }{ }^{1} \mathrm{H} \text { and } 77.16 \mathrm{ppm} \text { for }{ }^{13} \mathrm{C}\right)^{[1]}$. For assigning signal separation of ${ }^{1} \mathrm{H}-\mathrm{NMR}$-spektra the following abbreviations were used: $\mathrm{s}=$ singlet, $\mathrm{d}=$ doublet, $\mathrm{t}=$ triplet, $\mathrm{q}=$ quartet, $\mathrm{m}=$ multiplet, $\mathrm{dd}=$ double doublet, $\mathrm{ddd}=$ doublet of double doublets, $\mathrm{b}=$ bright, $\mathrm{Ar}-\mathrm{H}=$ aromatic. The signals of the ${ }^{13} \mathrm{C}$ NMR spectra were classified by the following abbreviations: $\mathrm{p}=$ primary $\left(\mathrm{RCH}_{3}\right), \mathrm{s}=$ secondary $\left(\mathrm{R}_{2} \mathrm{CH}_{2}\right), \mathrm{t}=$ tertiary $\left(\mathrm{R}_{3} \mathrm{CH}\right), \mathrm{q}=$ quaternary $\left(\mathrm{R}_{4} \mathrm{C}\right)$. The coupling constant $J$ was assigned in Hertz $[\mathrm{Hz}]$.

\section{Thin layer chromatography (TLC):}

The analytic TLC was carried out on TLC-alumina panels from Merck (Art.-Nr. 105554, silica gel $60 \mathrm{~F}_{254}$, layer thickness $0.2 \mathrm{~mm}$ ), The detection was carried out with an UV-lamp of the firm Heraeus, model Fluotest. Seebach reagent [Molybdo phosphoric acid (2.5 w.-\%), Cer(IV)sulfate tetrahydrate (1.0 w.-\%), $\mathrm{H}_{2} \mathrm{SO}_{4}$ conc. (6 w.- $\left.\%\right)$, water (90.5 w.-\%)] and 2,4-dinitrophenylhydrazine solution: 0.4 g 2,4-dinitrophenylhydrazine, $2 \mathrm{ml}$ conc. sulfuric acid, $10 \mathrm{ml}$ ethanol, $100 \mathrm{ml}$ dist. water were used as dipping reagents.

\section{Gas chromatography (GC)}

$\mathrm{GC}$ on achiral stationary phase $\left(\mathrm{GC}_{\mathrm{ASP}}\right)$ :

The measurements of the gas chromatograms were performed on a GC (Varian), model GC 3900 on capillary column Chrompak CP-Sil 5 CB (length 30 m, inner diameter $0.32 \mathrm{~mm}$, thickness of the film $0.33 \mu \mathrm{m}$ ), carrier gas helium, make-up gas nitrogen, injector: split $\left(250^{\circ} \mathrm{C}\right)$, detector: FID $\left(270{ }^{\circ} \mathrm{C}\right)$.

$\mathrm{GC}$ on chiral stationary phase $\left(\mathrm{GC}_{\mathrm{CSP}}\right)$ :

The measurements of the gas chromatograms were performed on a GC ( Varian), model GC 3800 on capillary column CP-Chirasil-Dex (length $25 \mathrm{~m}$, inner diameter $0.32 \mathrm{~mm}$, thickness 
of the film $0.25 \mu \mathrm{m}$ ), and Lipodex E (length $25 \mathrm{~m}$, inner diameter $0.25 \mathrm{~mm}$ ), carrier gas helium, make-up gas nitrogen, injector: split $\left(250^{\circ} \mathrm{C}\right)$, detector: FID $\left(270{ }^{\circ} \mathrm{C}\right)$.

HPLC was performed on Agilent 1100 Series using Diacel Chiralpak AS $(250 \times 4.6 \mathrm{~mm})$ or Diacel Chiracel OD $(250 \times 4.00 \mathrm{~mm}, 10 \mu \mathrm{m})$.

Rotational values were determined on a Perkin Elmer 241 Polarimeter at $\lambda=589 \mathrm{~nm}$ (sodium

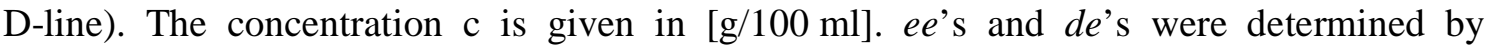
comparison with the racemic products obtained by application of DL-proline as catalyst.

\section{balances:}

analytical balance Sartorius Basic, balance Sartorius LC 620 S.

Solvents and chemicals:

Al chemicals were purchazed and uzed without further purification. The products were purified by flash-chromatography ${ }^{[2]}$ using silica gel 60 from SDS $(0.035-0.070 \mathrm{~mm})$ or Merck $(0.040-0.063 \mathrm{~mm})$ and sea sand (purified with acid and calcined) from Merck. The solvents were purified by distillation and were determined by volume. The composition of the moving phase of the TLC was also assigned volumetric.

The microwave-assisted reactions were conducted using a focused microwave unit (Discover ${ }^{\circledR}$ Reactor from CEM Corporation). The instrument consists of a continuous focused microwave power delivery system with operator selectable power output from 0-300 W. In all experiments the microwave power was held constant to ensure reproducibility. Reactions were performed in $10 \mathrm{~mL}$ glass vessels, which were sealed with a septum and locked into a pressure device, which controlled the pressure in the reaction vessel (maximum 10 bar). The specified reaction time corresponds to the total irradiation time. The temperature was monitored by an infrared temperature sensor positioned below the reaction vessel. The indicated temperature corresponds to the maximal temperature reached during each experiment. 
General Procedure for the Proline-Catalyzed $\alpha$-sulfamidation under Microwave Irradiation. Preparation of 2-Phenyl-2-(4'-toluene)sulfonylaminopropionaldehyde as a Detailed Representative Example. In a $10 \mathrm{~mL}$ vessel was placed 2-phenylpropionaldehyde (0.134 g, $1.00 \mathrm{mmol}, 1.0$ eq.), L-proline ( $2.30 \mathrm{mg}, 0.02 \mathrm{mmol}, 2 \mathrm{~mol} \%)$, chloramine-T $(0.423$ g, $1.50 \mathrm{mmol}, 1.5$ eq.), acetonitrile $(5 \mathrm{ml})$, and a magnetic stir bar. The vessel was sealed with a septum, placed into the MW cavity, and locked with the pressure device. Constant MW irradiation of $200 \mathrm{~W}$ as well as simultaneous air-cooling ( 0.7 bar, 10 psi) were used during the entire reaction time $(30 \mathrm{~min})$. After cooling to room temperature, the solvent was removed under reduced pressure, and the product was purified by column chromatography (silica gel, diethylether/pentane, $1: 2)$ to afford the $\alpha$-sulfamidated aldehyde as a white solid $(0.273 \mathrm{~g}$, $90 \%)$.

General Procedure for the Proline-Catalyzed $\alpha$-sulfamidation.

I) For reactions performed without microwave irradiation. General procedure (GP 1). In a 10 $\mathrm{mL}$ vessel was placed 2-phenylpropionaldehyde (0.134 g, $1.00 \mathrm{mmol}, 1.0$ eq.), L-proline (2.30 mg, $0.02 \mathrm{mmol}, 2 \mathrm{~mol} \%)$, chloramine-T ( $0.423 \mathrm{~g}, 1.50 \mathrm{mmol}, 1.5$ eq.), acetonitrile (5 $\mathrm{ml}$ ), and a magnetic stir bar. The reaction mixture was vigorously stirred at $70{ }^{\circ} \mathrm{C}$ in a preheated oil bath for $5 \mathrm{~h}$. After cooling to room temperature, the solvent was removed under reduced pressure, and the product was purified by column chromatography (silica gel, diethylether/pentane, 1:2) to afford the $\alpha$-sulfamidated aldehyde as a white solid $(0.258 \mathrm{~g}$, $85 \%)$.

II) For reactions performed under microwave irradiation. General procedure (GP 2). In a 10 $\mathrm{mL}$ vessel was placed the aldehyde ( $1.00 \mathrm{mmol}, 1.0$ eq. $)$, L-proline $(0.02 \mathrm{mmol}, 2 \mathrm{~mol} \%)$, chloramine-T (1.50 mmol, 1.5 eq.), acetonitrile $(5 \mathrm{ml})$, and a magnetic stir bar. The vessel was sealed with a septum, placed into the MW cavity, and locked with the pressure device. Constant MW irradiation of $200 \mathrm{~W}$ as well as simultaneous air-cooling ( 0.7 bar, $10 \mathrm{psi}$ ) were used during the entire reaction time $(30 \mathrm{~min})$. After cooling to room temperature, the solvent was removed under reduced pressure, and the product was purified by column chromatography (silica gel, diethylether/pentane) to afford the $\alpha$-sulfamidated aldehyde as a white solid.

III) For reactions performed at room temperature. General Procedure (GP 3). In a $10 \mathrm{~mL}$ vessel was placed the carbonyl compound ( $1.00 \mathrm{mmol}, 1.0 \mathrm{eq}$.), L-proline $(0.02 \mathrm{mmol}, 2 \mathrm{~mol}$ $\%)$, chloramine-T $(1.50 \mathrm{mmol}, 1.5$ eq. $)$, acetonitrile $(5 \mathrm{ml})$, and a magnetic stir bar. The reaction mixture was vigorously stirred at rt until TLC indicated complete conversion. The solvent was removed under reduced pressure, and the product was purified by column chromatography (silica gel, diethylether/pentane) to afford the sulfamidated carbonyl compound as a white solid. 


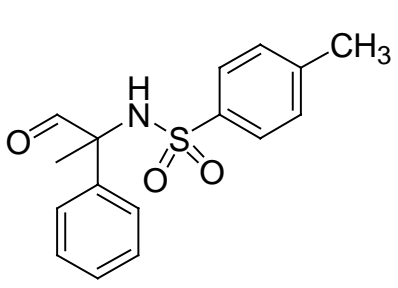

2-Phenyl-2-(4'-toluene)sulfonylaminopropionaldehyde 2a: The compound was synthesized following GP 1, using 2phenylpropionaldehyde $(0.134 \mathrm{~g}, 1.00 \mathrm{mmol})$, chloramine- $\mathrm{T}(0.423$ $\mathrm{g}, 1.50 \mathrm{mmol})$, and L-proline $(2.30 \mathrm{mg}, 0.02 \mathrm{mmol})$ in acetonitrile $(5 \mathrm{~mL})$ within $30 \mathrm{~min}$. Flash chromatography on silica (diethylether/pentane 1:1) delivered $0.273 \mathrm{~g}(0.90 \mathrm{mmol}, 90 \%)$ of a colorless solid. For reaction conditions, see Table $2 .-\mathrm{mp}=113{ }^{\circ} \mathrm{C} .-\mathrm{R}_{f}=0.22$ (diethylether/pentane, 1:2). ${ }^{1} \mathrm{H} \mathrm{NMR}\left(400 \mathrm{MHz}, \mathrm{CDCl}_{3}\right): \delta=1.91\left(\mathrm{~s}, 3 \mathrm{H}, \mathrm{CR}_{3} \mathrm{CH}_{3}\right), 2.36\left(\mathrm{~s}, 3 \mathrm{H}, \mathrm{C}^{4}{ }_{\mathrm{Ts}} \mathrm{CH}_{3}\right), 6.01(\mathrm{bs}$, $1 \mathrm{H}, \mathrm{NH}), 7.07$ (d, $\left.J=7.9 \mathrm{~Hz}, 2 \mathrm{H}, \mathrm{C}^{3} \mathrm{H}_{\mathrm{Ts}}\right), 7.14\left(\mathrm{td}, J=8.5,2.1 \mathrm{~Hz}, 2 \mathrm{H}, \mathrm{CH}_{\mathrm{Ph}}\right), 7.18-7.28$ $\left(\mathrm{m}, 3 \mathrm{H}, \mathrm{CH}_{\mathrm{Ph}}\right), 7.37$ (d, J=8.3 Hz, $\left.2 \mathrm{H}, \mathrm{C}^{2} \mathrm{H}_{\mathrm{Ts}}\right), 9.15$ (s, $\left.1 \mathrm{H}, \mathrm{CHO}\right) \mathrm{ppm} .-{ }^{13} \mathrm{C} \mathrm{NMR}$ $\left(100 \mathrm{MHz}, \mathrm{CDCl}_{3}\right): \delta=20.0\left(+, \mathrm{CR}_{3} \underline{\mathrm{CH}}_{3}\right), 21.4\left(+, \mathrm{C}^{4}{ }_{\mathrm{Ts}} \underline{\mathrm{CH}}_{3}\right), 66.8\left(\mathrm{q}, \mathrm{CR}_{4}\right), 126.6(+$,

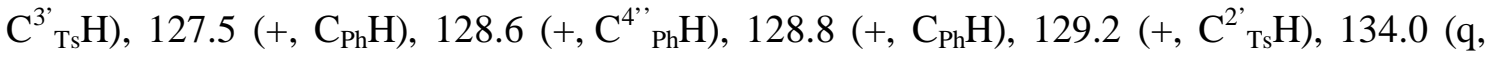
$\left.\mathrm{C}^{1}{ }^{\prime \prime} \mathrm{Ph}_{3}\right), 139.1$ (q, $\left.\mathrm{C}^{1}{ }_{\mathrm{Ts}} \mathrm{SO}_{2}\right), 142.8\left(\mathrm{q}, \mathrm{C}^{4}{ }_{\mathrm{Ts}} \mathrm{CH}_{3}\right), 194.4(+, \mathrm{CHO}) \mathrm{ppm} .-\mathrm{IR}(\mathrm{KBr}): v=$ $3250(\mathrm{~m}, \mathrm{v}[\mathrm{NH}]), 3067$ (w, v[ $\left.\left.\mathrm{CH}_{\mathrm{ar}}\right]\right), 2975$ (w, v[CH 3$\left.]\right), 2822,2718(\mathrm{w}, \mathrm{w}, \mathrm{v}[\mathrm{C}(\mathrm{O}) \mathrm{H}], 1745$ $(\mathrm{m}, \delta[\mathrm{NH}]), 1734(\mathrm{~m}, \mathrm{v}[\mathrm{CO}]), 1599,1583,1496\left(\mathrm{w}, \mathrm{w}, \mathrm{w}, \mathrm{v}\left[\mathrm{C}-\mathrm{C}_{\mathrm{ar}}\right]\right), 1443\left(\mathrm{~m}, \delta_{\mathrm{as}}\left[\mathrm{CH}_{3}\right]\right)$, $1366\left(\mathrm{~m}, \delta_{\mathrm{sy}}\left[\mathrm{CH}_{3}\right]\right), 1328\left(\mathrm{~m}, \mathrm{v}_{\mathrm{as}}\left[\mathrm{SO}_{2}\right], 1156\left(\mathrm{~m}, \mathrm{v}_{\mathrm{sy}}\left[\mathrm{SO}_{2}\right]\right), 817,767,\left(\mathrm{~m}, \mathrm{~m}, \delta\left[\mathrm{CH}_{\mathrm{ar}}\right]\right) \mathrm{cm}^{-1}\right.$. MS (EI, I, $70 \mathrm{eV}): \mathrm{m} / \mathrm{z}(\%)=274(100)\left[\mathrm{M}^{+}-\mathrm{CHO}\right], 155$ (34) $\left[\mathrm{C}_{7} \mathrm{H}_{7} \mathrm{SO}_{2}^{+}\right], 149$ (16) $\left[\mathrm{M}^{+}-\right.$ $\left.\mathrm{C}_{7} \mathrm{H}_{7} \mathrm{SO}_{2}\right], 104$ (6) $\left[\mathrm{C}_{8} \mathrm{H}_{8}^{+}\right], 91$ (50) $\left[\mathrm{C}_{7} \mathrm{H}_{7}{ }^{+}\right], 77$ (6) $\left[\mathrm{C}_{6} \mathrm{H}_{5}^{+}\right] .-\mathrm{HRMS}$ (I): calcd.: 274.0902 [M+-CHO], found: 274.0900. $-\mathrm{C}_{16} \mathrm{H}_{17} \mathrm{NO}_{3} \mathrm{~S}$ (303.38 g/mol), calcd.: C 63.35, H 5.65, N 4.62, found: C 63.71, H 5.68, N 4.64.

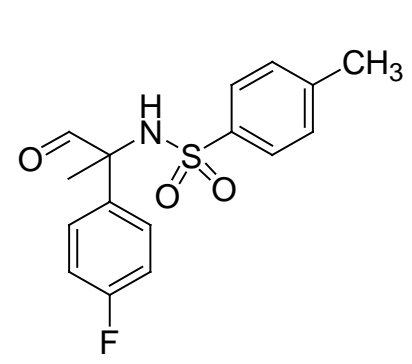

2-(4'-Fluorophenyl)-2-(4' '-toluene) sulfonylaminopropionaldehyde 2b: The compound was synthesized following GP 1, Method A, using 2-(4'-fluoropheny)propionaldehyde (0.152 g, $1.00 \mathrm{mmol})$, chloramine-T (0.423 g, $1.50 \mathrm{mmol})$, and L-proline $(2.30 \mathrm{mg}$, $0.02 \mathrm{mmol})$ in acetonitrile $(5 \mathrm{~mL})$ within $30 \mathrm{~min}$. Flash chromatography on silica (diethylether/pentane 1:2) delivered $0.273 \mathrm{~g}(0.85 \mathrm{mmol}, 85 \%)$ of a colorless solid. For reaction conditions, see Table 2. $-\mathrm{mp}=92$ ${ }^{\circ} \mathrm{C} .-\mathrm{R}_{f}=0.29$ (diethylether/pentane, 1:2). $-{ }^{1} \mathrm{H}$ NMR $\left(400 \mathrm{MHz}, \mathrm{CDCl}_{3}\right): \delta=1.83(\mathrm{~s}, 3 \mathrm{H}$, $\mathrm{CR}_{3} \mathrm{CH}_{3}$ ), 2.29 (s, $\left.3 \mathrm{H}, \mathrm{C}^{4}{ }_{\mathrm{Ts}} \mathrm{CH}_{3}\right), 5.94(\mathrm{bs}, 1 \mathrm{H}, \mathrm{NH}), 6.76\left(\mathrm{~d}, J=8.8 \mathrm{~Hz}, 2 \mathrm{H}, \mathrm{C}^{3} \mathrm{H}_{\mathrm{ar}}\right.$ ), 6.79 $\left(\mathrm{d}, J=8.6 \mathrm{~Hz}, 2 \mathrm{H}, \mathrm{C}^{3{ }^{\prime \prime}} \mathrm{H}_{\mathrm{Ts}}\right), 7.01\left(\mathrm{~d}, J=8.8 \mathrm{~Hz}, 2 \mathrm{H}, \mathrm{C}^{2} \mathrm{H}_{\mathrm{ar}}\right), 7.27(\mathrm{~d}, J=8.4 \mathrm{~Hz}, 2 \mathrm{H}$, $\left.\mathrm{C}^{2}{ }^{\prime \prime} \mathrm{H}_{\mathrm{Ts}}\right), 9.01(\mathrm{~s}, 1 \mathrm{H}, \mathrm{CHO}) \mathrm{ppm} .-{ }^{13} \mathrm{C} \mathrm{NMR}\left(100 \mathrm{MHz}, \mathrm{CDCl}_{3}\right): \delta=20.2\left(+, \mathrm{CR}_{3} \underline{C H}_{3}\right), 21.4$ $\left(+, \mathrm{C}^{4}{ }^{\prime \prime}{ }_{\mathrm{Ts}} \mathrm{CH}_{3}\right), 66.2\left(\mathrm{q}, \mathrm{CR}_{4}\right), 115.7\left(+, \mathrm{d}, J=22.0 \mathrm{~Hz}, \mathrm{C}^{3}{ }_{\mathrm{ar}} \mathrm{H}\right), 126.6\left(+, \mathrm{C}^{3{ }^{\prime \prime}}{ }_{\mathrm{Ts}} \mathrm{H}\right), 129.2(+$, 
$\left.\mathrm{C}^{2 ”}{ }_{\mathrm{Ts}} \mathrm{H}\right), 129.6\left(+, \mathrm{d}, J=8.9 \mathrm{~Hz}, \mathrm{C}^{2}{ }_{\mathrm{ar}} \mathrm{H}\right), 129.7$ (q, d, $\left.J=3.4 \mathrm{~Hz}, \mathrm{C}^{1}{ }_{\mathrm{ar}} \mathrm{CR}_{3}\right), 139.0$ (q, $\left.\mathrm{C}^{1 "}{ }_{\mathrm{Ts}} \mathrm{SO}_{2}\right), 144.0$ (q, $\left.\underline{\mathrm{C}}^{4 ”}{ }_{\mathrm{Ts}} \mathrm{CH}_{3}\right), 141.0$ (q, d, J= $\left.403.0 \mathrm{~Hz}, \mathrm{C}^{4}{ }_{\mathrm{ar}} \mathrm{F}\right), 193.9$ (+, CHO) ppm. - IR $(\mathrm{KBr}): v=3237(\mathrm{~m}, \mathrm{v}[\mathrm{NH}]), 3048\left(\mathrm{w}, \mathrm{v}\left[\mathrm{CH}_{\mathrm{ar}}\right]\right), 2972\left(\mathrm{w}, \mathrm{v}\left[\mathrm{CH}_{3}\right]\right), 2840,2724(\mathrm{w}, \mathrm{w}$, $\mathrm{v}[\mathrm{C}(\mathrm{O}) \mathrm{H}], 1737$ (m, v[CO]), 1602, 1509 (m, m, v[C-C $\mathrm{ar}]), 1449\left(\mathrm{~m}, \delta_{\mathrm{as}}\left[\mathrm{CH}_{3}\right]\right), 1382$ $\left(\mathrm{m}, \delta_{\text {sy }}\left[\mathrm{CH}_{3}\right]\right), 1326\left(\mathrm{~m}, \mathrm{v}_{\mathrm{as}}\left[\mathrm{SO}_{2}\right], 1164\left(\mathrm{~m}, \mathrm{v}_{\mathrm{sy}}\left[\mathrm{SO}_{2}\right]\right), 837,811\left(\mathrm{~m}, \mathrm{~m}, \delta\left[\mathrm{CH}_{\mathrm{ar}}\right]\right), 733,705(\mathrm{w}\right.$, w, v[CHal] $\mathrm{cm}^{-1}$. - MS (EI, I, $\left.70 \mathrm{eV}\right): \mathrm{m} / z(\%)=292(100)\left[\mathrm{M}^{+}-\mathrm{CHO}\right], 155(24)\left[\mathrm{C}_{7} \mathrm{H}_{7} \mathrm{SO}_{2}{ }^{+}\right]$, 122 (12) $\left[\mathrm{C}_{8} \mathrm{H}_{8} \mathrm{~F}^{+}\right], 91$ (59) $\left[\mathrm{C}_{7} \mathrm{H}_{7}^{+}\right]$. - HRMS (I): calcd.: 292.0808 [M+CHO], found: 292.0811. $-\mathrm{C}_{16} \mathrm{H}_{16} \mathrm{FNO}_{3} \mathrm{~S}(321.37 \mathrm{~g} / \mathrm{mol})$, calcd.: C 59.80, H 5.02, N 4.36, found: C 60.01, H 5.12, N 4.42 .

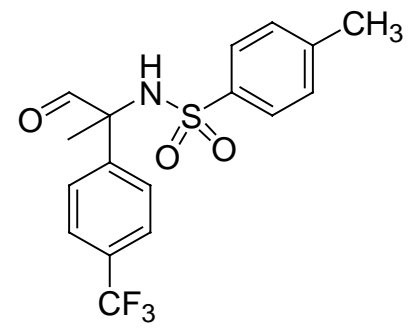

2-(4'-Trifluoromethylphenyl)-2-(4',toluene)sulfonylaminopropionaldehyde 2c: The compound was synthesized following GP 1, using 2-(4'trifluoromethylphenyl)propionaldehyde $(0.202 \mathrm{~g}, 1.00 \mathrm{mmol})$, chloramine-T $(0.423 \mathrm{~g}, 1.50 \mathrm{mmol})$, and L-proline $(2.30 \mathrm{mg}$, $0.02 \mathrm{mmol})$ in acetonitrile $(5 \mathrm{~mL})$ within $30 \mathrm{~min}$. Flash chromatography on silica (diethylether/pentane 1:2) delivered $0.293 \mathrm{~g}(0.79 \mathrm{mmol}, 79 \%)$ of a colorless solid. For reaction conditions, see Table $2 .-\mathrm{mp}=81{ }^{\circ} \mathrm{C} .-\mathrm{R}_{f}=0.32$ (diethylether/pentane, 1:2). ${ }^{1} \mathrm{H} \mathrm{NMR}\left(400 \mathrm{MHz}, \mathrm{CDCl}_{3}\right): \delta=1.83\left(\mathrm{~s}, 3 \mathrm{H}, \mathrm{CR}_{3} \mathrm{CH}_{3}\right), 2.24\left(\mathrm{~s}, 3 \mathrm{H}, \mathrm{C}^{4}{ }_{\mathrm{Ts}} \mathrm{CH}_{3}\right), 6.21(\mathrm{bs}$, $1 \mathrm{H}, \mathrm{NH}), 6.93\left(\mathrm{~d}, J=8.0 \mathrm{~Hz}, 1 \mathrm{H}, \mathrm{C}^{3}{ }^{\prime \prime} \mathrm{H}_{\mathrm{Ts}}\right), 7.13\left(\mathrm{~d}, J=8.2 \mathrm{~Hz}, 1 \mathrm{H}, \mathrm{C}^{2} \mathrm{H}_{\mathrm{ar}}\right), 7.22(\mathrm{~d}, J=8.3$ $\mathrm{Hz}, 1 \mathrm{H}, \mathrm{C}^{3} \mathrm{H}_{\mathrm{ar}}$ ), 7.27 (d, J = 8.3 Hz, $1 \mathrm{H}, \mathrm{C}^{2}{ }^{\prime \prime} \mathrm{H}_{\mathrm{Ts}}$ ), 9.07 (s, $\left.1 \mathrm{H}, \mathrm{CHO}\right)$ ppm. $-{ }^{13} \mathrm{C} \mathrm{NMR}$ $\left(100 \mathrm{MHz}, \mathrm{CDCl}_{3}\right): \delta=20.1\left(+, \mathrm{CR}_{3} \underline{\mathrm{CH}}_{3}\right), 21.2\left(+, \mathrm{C}^{4}{ }_{\mathrm{Ts}} \underline{\mathrm{CH}}_{3}\right), 66.5$ (q, $\left.\mathrm{CR}_{4}\right), 123.7$ (q, q, $J=$ $\left.272.4 \mathrm{~Hz}, \mathrm{C}^{4^{\prime}}{ }_{\mathrm{ar}} \underline{\mathrm{CF}}_{3}\right), 125.5$ (q, q, $\left.J=3.7 \mathrm{~Hz}, \mathrm{C}^{3{ }^{\prime}}{ }_{\mathrm{ar}} \mathrm{H}\right), 126.5\left(+, \mathrm{C}^{2}{ }^{\prime \prime}{ }_{\mathrm{Ts}} \mathrm{H}\right), 128.1\left(+, \mathrm{C}^{2}{ }_{\mathrm{ar}} \mathrm{H}\right)$,

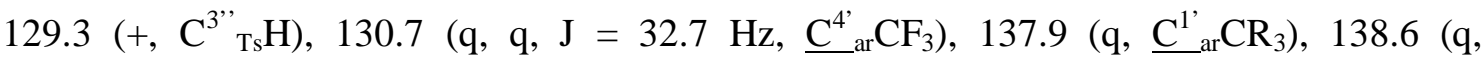
$\left.\mathrm{C}^{1 "}{ }_{\mathrm{Ts}} \mathrm{SO}_{2}\right), 143.2$ (q, $\left.\underline{\mathrm{C}}^{4 ”}{ }_{\mathrm{Ts}} \mathrm{CH}_{3}\right), 193.9$ (+, CHO) ppm. - IR $(\mathrm{KBr}): \mathrm{v}=3236(\mathrm{~m}, \mathrm{v}[\mathrm{NH}]), 3068$ (w, v[ $\left[\mathrm{CH}_{\mathrm{ar}}\right]$ ), 2978 (w, v[CH 3$\left.]\right), 2848,2733$ (w, w, v[C(O)H], 1741 (m, v[CO]), 1597, 1496 $\left(\mathrm{m}, \mathrm{m}, \mathrm{v}\left[\mathrm{C}-\mathrm{C}_{\mathrm{ar}}\right]\right), 1409\left(\mathrm{~m}, \delta_{\mathrm{as}}\left[\mathrm{CH}_{3}\right]\right), 1369\left(\mathrm{w}, \delta_{\mathrm{sy}}\left[\mathrm{CH}_{3}\right]\right), 1327\left(\mathrm{~m}, \mathrm{v}_{\mathrm{as}}\left[\mathrm{SO}_{2}\right], 1148(\mathrm{~m}\right.$, $\left.v_{\text {sy }}\left[\mathrm{SO}_{2}\right]\right), 840,811\left(\mathrm{~m}, \mathrm{~m}, \delta\left[\mathrm{CH}_{\mathrm{ar}}\right]\right), 782,711\left(\mathrm{~m}, \mathrm{~m}, \mathrm{v}[\mathrm{CHal}] \mathrm{cm}^{-1}\right.$. - MS (EI, I, $\left.70 \mathrm{eV}\right)$ : $m / z(\%)=342(100)\left[\mathrm{M}^{+}-\mathrm{CHO}\right], 172(18), 155(92)\left[\mathrm{C}_{7} \mathrm{H}_{7} \mathrm{SO}_{2}^{+}\right], 91(82)\left[\mathrm{C}_{7} \mathrm{H}_{7}^{+}\right], 77$ (2) $\left[\mathrm{C}_{6} \mathrm{H}_{5}^{+}\right]$. $-\mathrm{HRMS}$ (I): calcd.: $371.0803\left[\mathrm{M}^{+}\right]$, found: 371.0808. - $\mathrm{C}_{17} \mathrm{H}_{16} \mathrm{~F}_{3} \mathrm{NO}_{3} \mathrm{~S}$ (371.37 g/mol), calcd.: C 54.98, H 4.34, N 3.77, found: C 55.13, H 4.40, N 3.70. 
2-(4'-Nitrophenyl)-2-(4' '-toluene) sulfonylaminopropionaldehyde

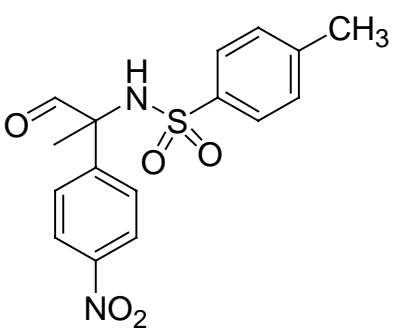

2d: The compound was synthesized following GP 1, using 2-(4'nitrophenyl)propionaldehyde (0.197 g, $1.00 \mathrm{mmol})$, chloramine-T (0.423 g, $1.50 \mathrm{mmol})$, and L-proline $(2.30 \mathrm{mg}, 0.02 \mathrm{mmol})$ in acetonitrile $(5 \mathrm{~mL})$ within $30 \mathrm{~min}$. Flash chromatography on silica (diethylether/pentane 1:1) delivered $0.310 \mathrm{mg}(0.89 \mathrm{mmol}, 89 \%)$ of a slightly yellow solid. For reaction conditions, see Table $2 .-\mathrm{mp}=133{ }^{\circ} \mathrm{C} .-\mathrm{R}_{f}=0.25$ (diethylether/pentane 1:1). - ${ }^{1} \mathrm{H}$ NMR (400 MHz, $\mathrm{CDCl}_{3}$ ): $\delta=1.85$ (s, $3 \mathrm{H}, \mathrm{CR}_{3} \mathrm{CH}_{3}$ ), 2.29 (s, $\left.3 \mathrm{H}, \mathrm{C}^{4}{ }_{\mathrm{Ts}} \mathrm{CH}_{3}\right), 6.14$ (bs, $\left.1 \mathrm{H}, \mathrm{NH}\right), 7.01\left(\mathrm{~d}, J=8.0 \mathrm{~Hz}, 2 \mathrm{H}, \mathrm{C}^{3}{ }^{\prime \prime} \mathrm{H}_{\mathrm{Ts}}\right), 7.27(\mathrm{~d}, J=9.0 \mathrm{~Hz}, 2 \mathrm{H}$, $\left.\mathrm{C}^{3{ }^{\prime}} \mathrm{H}_{\mathrm{ar}}\right), 7.32\left(\mathrm{~d}, J=8.3 \mathrm{~Hz}, 2 \mathrm{H}, \mathrm{C}^{2{ }^{\prime \prime}} \mathrm{H}_{\mathrm{Ts}}\right), 7.93\left(\mathrm{~d}, J=9.0 \mathrm{~Hz}, 2 \mathrm{H}, \mathrm{C}^{2} \mathrm{H}_{\mathrm{ar}}\right), 9.10(\mathrm{~s}, 1 \mathrm{H}$, $\mathrm{CHO})$ ppm. $-{ }^{13} \mathrm{C}$ NMR $\left(100 \mathrm{MHz}, \mathrm{CDCl}_{3}\right): \delta=20.3\left(+, \mathrm{CR}_{3} \underline{\mathrm{CH}}_{3}\right), 21.4\left(+, \mathrm{C}^{4}{ }_{\mathrm{Ts}} \underline{\mathrm{C}} \mathrm{H}_{3}\right), 66.7$ $\left(\mathrm{q}, \mathrm{CR}_{4}\right), 123.7\left(+, \mathrm{C}^{3}{ }_{\text {ar }} \mathrm{H}\right), 126.6\left(+, \mathrm{C}^{3{ }^{3 \prime}}{ }_{\mathrm{Ts}} \mathrm{H}\right), 128.7\left(+, \mathrm{C}^{2}{ }^{\prime}{ }_{\mathrm{ar}} \mathrm{H}\right), 129.4\left(+, \mathrm{C}^{2{ }^{\prime \prime}}{ }_{\mathrm{Ts}} \mathrm{H}\right), 138.7$ (q, $\left.\mathrm{C}^{1 "}{ }_{\mathrm{Ts}} \mathrm{SO}_{2}\right), 141.6\left(\mathrm{q}, \mathrm{C}^{1}{ }_{\mathrm{ar}} \mathrm{CR}_{3}\right), 143.7$ (q, $\left.\mathrm{C}^{4 \prime}{ }_{\mathrm{Ts}} \mathrm{CH}_{3}\right), 147.7$ (q, $\left.\mathrm{C}^{4}{ }_{\mathrm{ar}} \mathrm{NO}_{2}\right), 193.3$ (+, $\left.\mathrm{CHO}\right)$ ppm. - IR (KBr): v = $3240(\mathrm{~m}, v[\mathrm{NH}]), 3116\left(\mathrm{w}, \mathrm{v}\left[\mathrm{CH}_{\mathrm{ar}}\right]\right), 2982\left(\mathrm{w}, \mathrm{v}\left[\mathrm{CH}_{3}\right]\right), 2850,2736(\mathrm{w}$, w, v[C(O)H], $1741(\mathrm{~m}, \delta[\mathrm{NH}]), 1728(\mathrm{~m}, \mathrm{v}[\mathrm{CO}]), 1598\left(\mathrm{~m}, \mathrm{v}\left[\mathrm{C}-\mathrm{C}_{\mathrm{ar}}\right]\right), 1517\left(\mathrm{~m}, \mathrm{v}\left[\mathrm{NO}_{2}\right]\right)$, $1450\left(\mathrm{~m}, \delta_{\mathrm{as}}\left[\mathrm{CH}_{3}\right]\right), 1389\left(\mathrm{~m}, \delta_{\text {sy }}\left[\mathrm{CH}_{3}\right]\right), 1348\left(\mathrm{~m}, \mathrm{v}\left[\mathrm{NO}_{2}\right]\right), 1325\left(\mathrm{~m}, \mathrm{v}_{\mathrm{as}}\left[\mathrm{SO}_{2}\right], 1147(\mathrm{~m}\right.$, $\left.v_{\mathrm{sy}}\left[\mathrm{SO}_{2}\right]\right), 856,814\left(\mathrm{~m}, \mathrm{~m}, \delta\left[\mathrm{CH}_{\mathrm{ar}}\right]\right) \mathrm{cm}^{-1}$. - MS (EI, I, $\left.70 \mathrm{eV}\right): \mathrm{m} / z(\%)=319(100)\left[\mathrm{M}^{+}-\right.$ CHO], 155 (58) $\left[\mathrm{C}_{7} \mathrm{H}_{7} \mathrm{SO}_{2}{ }^{+}\right], 91$ (48) $\left[\mathrm{C}_{7} \mathrm{H}_{7}{ }^{+}\right], 77$ (3) $\left[\mathrm{C}_{6} \mathrm{H}_{5}{ }^{+}\right] .-$HRMS (I): calcd:: 319.0753 [M+-CHO], found: 319.0755. $-\mathrm{C}_{16} \mathrm{H}_{16} \mathrm{~N}_{2} \mathrm{O}_{5} \mathrm{~S}$ (348.37 g/mol), calcd.: C 55.16, H 4.63, N 8.04, found: 55.24, H 4.61, N 7.95.

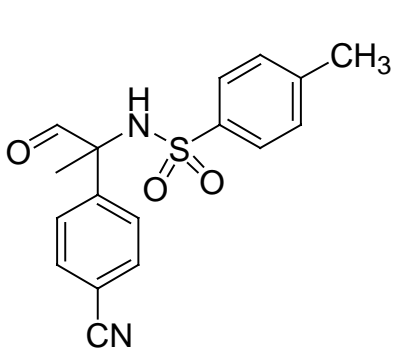

2-(4'-Cyanophenyl)-2-(4',-toluene) sulfonylaminopropionaldehyde 2e: The compound was synthesized following GP 1, using 2-(4'cyanophenyl)propionaldehyde (0.159 g, $1.00 \mathrm{mmol})$, chloramine-T (0.423 g, $1.50 \mathrm{mmol})$, and L-proline $(2.30 \mathrm{mg}, 0.02 \mathrm{mmol})$ in acetonitrile $(5 \mathrm{~mL})$ within $30 \mathrm{~min}$. Flash chromatography on silica (diethylether/pentane 1:1) delivered $0.282 \mathrm{~g}(0.86 \mathrm{mmol}, 86 \%)$ of a colorless solid. For reaction conditions, see Table 2. $-\mathrm{mp}=117{ }^{\circ} \mathrm{C} . \quad-\mathrm{R}_{f}=0.20$ (diethylether/pentane 1:1). $-{ }^{1} \mathrm{H}$ NMR $\left(400 \mathrm{MHz}, \mathrm{CDCl}_{3}\right): \delta=1.82\left(\mathrm{~s}, 3 \mathrm{H}, \mathrm{CR}_{3} \mathrm{CH}_{3}\right.$ ), $2.32(\mathrm{~s}$, $\left.3 \mathrm{H}, \mathrm{C}^{4}{ }_{\mathrm{Ts}} \mathrm{CH}_{3}\right), 6.08$ (bs, $\left.1 \mathrm{H}, \mathrm{NH}\right), 7.05\left(\mathrm{~d}, J=8.0 \mathrm{~Hz}, 2 \mathrm{H}, \mathrm{C}^{3{ }^{\prime \prime}} \mathrm{H}_{\mathrm{Ts}}\right), 7.22(\mathrm{~d}, J=8.7 \mathrm{~Hz}, 2$ $\left.\mathrm{H}, \mathrm{C}^{3} \mathrm{H}_{\mathrm{ar}}\right), 7.32\left(\mathrm{~d}, J=8.3 \mathrm{~Hz}, 1 \mathrm{H}, \mathrm{C}^{2}{ }^{\prime \prime} \mathrm{H}_{\mathrm{Ts}}\right), 7.41\left(\mathrm{~d}, J=8.7 \mathrm{~Hz}, 1 \mathrm{H}, \mathrm{C}^{2} \mathrm{H}_{\mathrm{ar}}\right), 9.07(\mathrm{~s}, 1 \mathrm{H}$, $\mathrm{CHO})$ ppm. $-{ }^{13} \mathrm{C} \mathrm{NMR}\left(100 \mathrm{MHz}, \mathrm{CDCl}_{3}\right): \delta=20.0\left(+, \mathrm{CR}_{3} \underline{\mathrm{CH}}_{3}\right), 21.5\left(+, \mathrm{C}^{4}{ }_{\mathrm{Ts}} \underline{\mathrm{CH}}_{3}\right), 66.7$

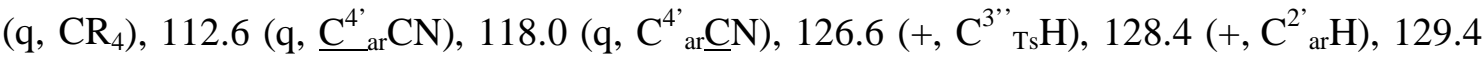




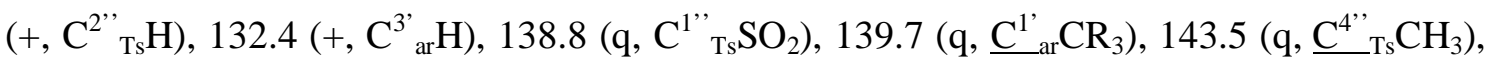
$193.4(+, \mathrm{CHO})$ ppm. - IR (KBr): v = $3227(\mathrm{~m}, \mathrm{v}[\mathrm{NH}]), 3045\left(\mathrm{w}, \mathrm{v}\left[\mathrm{CH}_{\mathrm{ar}}\right]\right), 2989\left(\mathrm{w}, \mathrm{v}\left[\mathrm{CH}_{3}\right]\right)$, 2850, $2721(\mathrm{w}, \mathrm{w}, \mathrm{v}[\mathrm{C}(\mathrm{O}) \mathrm{H}], 2229(\mathrm{~m}, \mathrm{v}[\mathrm{CN}]), 1740(\mathrm{~m}, \mathrm{v}[\mathrm{CO}]), 1597,1505$ (w, w, v[C$\left.\left.\mathrm{C}_{\mathrm{ar}}\right]\right), 1438\left(\mathrm{~m}, \delta_{\mathrm{as}}\left[\mathrm{CH}_{3}\right]\right), 1384\left(\mathrm{~m}, \delta_{\text {sy }}\left[\mathrm{CH}_{3}\right]\right), 1325\left(\mathrm{~m}, \mathrm{v}_{\mathrm{as}}\left[\mathrm{SO}_{2}\right], 1150\left(\mathrm{~m}, \mathrm{v}_{\mathrm{sy}}\left[\mathrm{SO}_{2}\right]\right), 836,810\right.$ $\left(\mathrm{m}, \mathrm{m}, \delta\left[\mathrm{CH}_{\mathrm{ar}}\right]\right) \mathrm{cm}^{-1}$. - MS (EI, I, $\left.70 \mathrm{eV}\right): \mathrm{m} / z(\%)=299(100)\left[\mathrm{M}^{+}-\mathrm{CHO}\right], 155$ (79) $\left[\mathrm{C}_{7} \mathrm{H}_{7} \mathrm{SO}_{2}^{+}\right], 129$ (11) $\left[\mathrm{C}_{9} \mathrm{H}_{7} \mathrm{~N}^{+}\right], 91$ (46) $\left[\mathrm{C}_{7} \mathrm{H}_{7}^{+}\right]$. - HRMS (I): calcd.: 299.0854 [M $\left.{ }^{+}-\mathrm{CHO}\right]$, found: 299.0852. $-\mathrm{C}_{17} \mathrm{H}_{16} \mathrm{~N}_{2} \mathrm{O}_{3} \mathrm{~S}$ (328.39 g/mol), calcd.: C 62.18, H 4.91, N 8.53, found: C 62.18, H 5.01, N 8.43.

2-(4'-Methylphenyl)-2-(4''-toluene) sulfonylaminopropionaldehyde<smiles>Cc1ccc(C(C=O)(NS(=O)(=O)c2ccc(C)cc2)c2ccc(C)cc2)cc1</smiles>

2f: The compound was synthesized following GP 1, using 2-(4'methyl)propionaldehyde $(0.148 \mathrm{~g}, 1.00 \mathrm{mmol})$, chloramine-T (0.423 g, $1.50 \mathrm{mmol})$, and L-proline $(2.30 \mathrm{mg}, 0.02 \mathrm{mmol})$ in acetonitrile $(5 \mathrm{~mL})$ within $30 \mathrm{~min}$. Flash chromatography on silica (diethylether/pentane 1:2) delivered $0.231 \mathrm{~g}(0.73 \mathrm{mmol}, 73 \%)$ of a colorless solid. For reaction conditions, see Table 2. $-\mathrm{mp}=121{ }^{\circ} \mathrm{C} .-\mathrm{R}_{f}=0.25$ (diethylether/pentane, 1:2). ${ }^{1} \mathrm{H}$ NMR (400 MHz, $\mathrm{CDCl}_{3}$ ): $\delta=1.80$ (s, $3 \mathrm{H}, \mathrm{CR}_{3} \mathrm{CH}_{3}$ ), 2.21 (s, $3 \mathrm{H}, \mathrm{C}^{4}{ }_{\mathrm{Ts}} \mathrm{CH}_{3}$ ), 2.28 (s, $\left.3 \mathrm{H}, \mathrm{C}^{4}{ }_{\mathrm{ar}} \mathrm{CH}_{3}\right), 5.89$ (bs, $\left.1 \mathrm{H}, \mathrm{NH}\right), 6.90$ (s, $4 \mathrm{H}, \mathrm{CH}_{\mathrm{ar}}$ ), 6.97 (d, $\left.J=8.5 \mathrm{~Hz}, 2 \mathrm{H}, \mathrm{C}^{3{ }^{\prime \prime}} \mathrm{H}_{\mathrm{Ts}}\right), 7.27\left(\mathrm{~d}, J=8.3 \mathrm{~Hz}, 2 \mathrm{H}, \mathrm{C}^{2}{ }^{\prime \prime} \mathrm{H}_{\mathrm{Ts}}\right), 9.03$ (s, $\left.1 \mathrm{H}, \mathrm{CHO}\right)$ ppm. ${ }^{13} \mathrm{C}$ NMR $\left(100 \mathrm{MHz}, \mathrm{CDCl}_{3}\right): \delta=19.8\left(+, \mathrm{CR}_{3} \underline{\mathrm{CH}_{3}}\right), 21.0\left(+, \mathrm{C}^{4}{ }_{\mathrm{ar}} \underline{\mathrm{CH}_{3}}\right), 21.4\left(+, \mathrm{C}^{4}{ }^{\prime \prime}{ }_{\mathrm{Ts}} \mathrm{CH}_{3}\right)$,

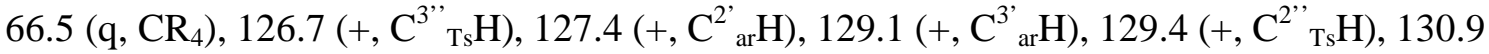
(q, $\left.\mathrm{C}^{4}{ }_{a r} \mathrm{CH}_{3}\right), 138.7$ (q, $\left.\mathrm{C}^{1}{ }_{a r} \mathrm{CR}_{3}\right), 139.1$ (q, $\left.\mathrm{C}^{1}{ }_{\mathrm{Ts}} \mathrm{SO}_{2}\right), 142.6$ (q, $\left.\mathrm{C}^{4}{ }^{\prime \prime}{ }_{\mathrm{ss}} \mathrm{CH}_{3}\right), 194.3$ (+, $\left.\mathrm{CHO}\right)$ ppm. - IR (KBr): v = $3248(\mathrm{~m}, \mathrm{v}[\mathrm{NH}]), 3068,3059,3027$ (m, m, m, v[CHar $), 2980,2920$ (m, $\left.\mathrm{m}, \mathrm{v}\left[\mathrm{CH}_{3}\right]\right), 2840,2714(\mathrm{~m}, \mathrm{w}, \mathrm{v}[\mathrm{C}(\mathrm{O}) \mathrm{H}], 1734$ (s, v[CO]), 1666, 1597, 1508 (m, m, m, v[C$\left.\left.\mathrm{C}_{\mathrm{ar}}\right]\right), 1397\left(\mathrm{~m}, \delta_{\mathrm{as}}\left[\mathrm{CH}_{3}\right]\right), 1358\left(\mathrm{~m}, \delta_{\mathrm{sy}}\left[\mathrm{CH}_{3}\right]\right), 1328\left(\mathrm{~s}, \mathrm{v}_{\mathrm{as}}\left[\mathrm{SO}_{2}\right], 1161\left(\mathrm{~s}, \mathrm{v}_{\mathrm{sy}}\left[\mathrm{SO}_{2}\right]\right), 844,818\right.$ $\left(\mathrm{m}, \mathrm{m}, \delta\left[\mathrm{CH}_{\mathrm{ar}}\right]\right) \mathrm{cm}^{-1} .-\mathrm{MS}(\mathrm{EI}, \mathrm{I}, 70 \mathrm{eV}): \mathrm{m} / z(\%)=288(88)\left[\mathrm{M}^{+}-\mathrm{CHO}\right], 171$ (23), 155 (41) $\left[\mathrm{C}_{7} \mathrm{H}_{7} \mathrm{SO}_{2}^{+}\right], 91$ (100) $\left[\mathrm{C}_{7} \mathrm{H}_{7}^{+}\right], 77$ (5) $\left[\mathrm{C}_{6} \mathrm{H}_{5}{ }^{+}\right]$. - HRMS (I): calcd.: $317.1086\left[\mathrm{M}^{+}\right]$, found: 317.1092. $-\mathrm{C}_{17} \mathrm{H}_{19} \mathrm{NO}_{3} \mathrm{~S}$ (317.40 g/mol), calcd.: C 64.33, H 6.03, N 4.41, found: C 64.23, H 5.97, N 4.58.

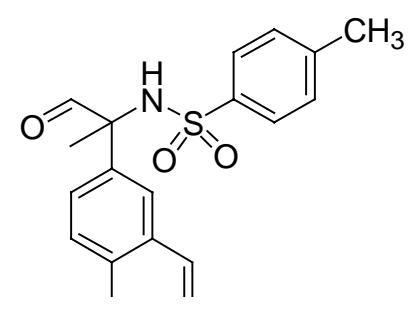

2-Napht-2-yl-2-(4'-toluene)sulfonylaminopropionaldehyde $\mathbf{2 g}$ : The compound was synthesized following GP 1, using 2-Naphth-2- 
ylpropionaldehyde $(0.184 \mathrm{~g}, 1.00 \mathrm{mmol})$, chloramine-T $(0.423 \mathrm{~g}, 1.50 \mathrm{mmol})$, and L-proline $(2.30 \mathrm{mg}, 0.02 \mathrm{mmol})$ in acetonitrile $(5 \mathrm{~mL})$ within $30 \mathrm{~min}$. Flash chromatography on silica (diethylether/pentane 1:2) delivered $0.321 \mathrm{~g}(0.91 \mathrm{mmol}, 91 \%)$ of a colorless solid. For reaction conditions, see Table $2 .-\mathrm{mp}=135{ }^{\circ} \mathrm{C} .-\mathrm{R}_{f}=0.35$ (diethylether/pentane 1:2). ${ }^{1} \mathrm{H}$ NMR (400 MHz, $\left.\mathrm{CDCl}_{3}\right): \delta=1.98\left(\mathrm{~s}, 3 \mathrm{H}, \mathrm{CR}_{3} \mathrm{CH}_{3}\right), 2.06\left(\mathrm{~s}, 3 \mathrm{H}, \mathrm{C}^{4}{ }_{\mathrm{Ts}} \mathrm{CH}_{3}\right), 6.02(\mathrm{bs}$, $1 \mathrm{H}, \mathrm{NH}), 6.66\left(\mathrm{~d}, J=8.0 \mathrm{~Hz}, 2 \mathrm{H}, \mathrm{C}^{3} \mathrm{H}_{\mathrm{Ts}}\right), 7.01\left(\mathrm{dd}, J=8.6,2.0 \mathrm{~Hz}, 1 \mathrm{H}, \mathrm{CH}_{\mathrm{Naph}}\right), 7.13(\mathrm{~d}$, $\left.J=8.3 \mathrm{~Hz}, 2 \mathrm{H}, \mathrm{C}^{2} \mathrm{H}_{\mathrm{Ts}}\right), 7.42\left(\mathrm{dd}, J=7.8,2.5 \mathrm{~Hz}, 1 \mathrm{H}, \mathrm{CH}_{\mathrm{Naph}}\right), 7.42(\mathrm{t}, J=6.8 \mathrm{~Hz}, 1 \mathrm{H}$, $\left.\mathrm{CH}_{\mathrm{Naph}}\right), 7.46\left(\mathrm{dd}, J=4.8,1.7 \mathrm{~Hz}, 1 \mathrm{H}, \mathrm{CH}_{\mathrm{Naph}}\right), 7.49$ (d, J = 8.7 Hz, $\left.1 \mathrm{H}, \mathrm{CH}_{\mathrm{Naph}}\right), 7.58$ (dd, $J$ = 7.0, $\left.2.1 \mathrm{~Hz}, 1 \mathrm{H}, \mathrm{CH}_{\mathrm{Naph}}\right), 7.68$ (dd, J = 7.0, $\left.2.1 \mathrm{~Hz}, 1 \mathrm{H}, \mathrm{CH}_{\mathrm{Naph}}\right), 9.09$ (s, $1 \mathrm{H}, \mathrm{CHO}$ ) ppm. $-{ }^{13} \mathrm{C}$ NMR $\left(100 \mathrm{MHz}, \mathrm{CDCl}_{3}\right): \delta=20.0\left(+, \mathrm{CR}_{3} \underline{\mathrm{CH}}_{3}\right), 21.2\left(+, \mathrm{C}^{4}{ }_{\mathrm{Ts}} \underline{\mathrm{CH}}_{3}\right), 66.7\left(\mathrm{q}, \mathrm{CR}_{4}\right)$, $124.4\left(+, \mathrm{CH}_{\mathrm{Naph}}\right), 126.5\left(+, \mathrm{CH}_{\mathrm{Naph}}\right), 126.5\left(+, \mathrm{C}^{3}{ }_{\mathrm{Ts}} \mathrm{H}\right), 127.0\left(+, \mathrm{CH}_{\mathrm{Naph}}\right), 127.4\left(+, \mathrm{CH}_{\mathrm{Naph}}\right)$, $127.7\left(+, \mathrm{CH}_{\mathrm{Naph}}\right), 128.2\left(+, \mathrm{CH}_{\mathrm{Naph}}\right), 128.6\left(+, \mathrm{CH}_{\mathrm{Naph}}\right), 128.9\left(+, \mathrm{C}^{2}{ }_{\mathrm{Ts}} \mathrm{H}\right), 130.5\left(\mathrm{q}, \mathrm{C}^{9++}{ }_{\mathrm{Naph}}\right)$, $132.8\left(\mathrm{C}^{10++}{ }_{\text {Naph }}\right), 133.0$ (q, $\left.\underline{\mathrm{C}}^{1}{ }_{\mathrm{Naph}} \mathrm{CR}_{3}\right), 138.7$ (q, $\left.\mathrm{C}^{1}{ }_{\mathrm{Ts}} \mathrm{SO}_{2}\right), 142.6\left(\mathrm{q}, \mathrm{C}^{4^{\prime}}{ }_{\mathrm{Ts}} \mathrm{CH}_{3}\right), 194.1(+$, CHO) ppm. - IR (KBr): v = $3271(\mathrm{~m}, \mathrm{v}[\mathrm{NH}]), 3064\left(\mathrm{w}, \mathrm{v}\left[\mathrm{CH}_{\mathrm{ar}}\right]\right), 2978\left(\mathrm{w}, \mathrm{v}\left[\mathrm{CH}_{3}\right]\right), 2834$, $2733(\mathrm{w}, \mathrm{w}, \mathrm{v}[\mathrm{C}(\mathrm{O}) \mathrm{H}], 1723$ (m, v[CO]), 1596, 1509, 1494 (m, w, w, v[C-Car]), 1448 (w, $\left.\delta_{\mathrm{as}}\left[\mathrm{CH}_{3}\right]\right), 1369\left(\mathrm{~m}, \delta_{\mathrm{sy}}\left[\mathrm{CH}_{3}\right]\right), 1325\left(\mathrm{~m}, \mathrm{v}_{\mathrm{as}}\left[\mathrm{SO}_{2}\right], 1154\left(\mathrm{~m}, \mathrm{v}_{\mathrm{sy}}\left[\mathrm{SO}_{2}\right]\right), 857,812,750(\mathrm{~m}, \mathrm{~m}, \mathrm{~m}\right.$, $\left.\delta\left[\mathrm{CH}_{\mathrm{ar}}\right]\right) \mathrm{cm}^{-1}$. - MS (EI, I, $\left.70 \mathrm{eV}\right): \mathrm{m} / z(\%)=324(100)\left[\mathrm{M}^{+}-\mathrm{CHO}\right], 169$ (5), 155 (21) $\left[\mathrm{C}_{7} \mathrm{H}_{7} \mathrm{SO}_{2}^{+}\right], 127$ (4) $\left[\mathrm{C}_{10} \mathrm{H}_{7}^{+}\right], 91$ (10) $\left[\mathrm{C}_{7} \mathrm{H}_{7}^{+}\right]$. - HRMS (I): calcd.: $353.1086\left[\mathrm{M}^{+}\right]$, found: 353.1086. $-\mathrm{C}_{20} \mathrm{H}_{19} \mathrm{NO}_{3} \mathrm{~S}$ (353.43 g/mol), calcd.: C 67.97, H 5.42, N 3.96, found: C 67.85, H 5.49, N 3.97.

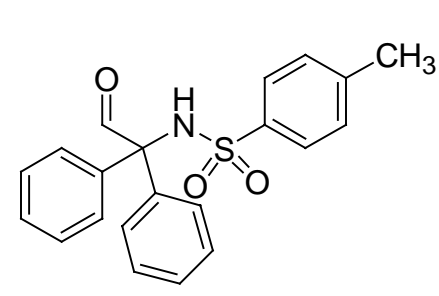

2,2-Diphenyl-2-(4'-toluene)sulfonylaminoacetaldehyde $\mathbf{2 h}$ : The compound was synthesized following GP 1, using 2,2diphenylacetaldehyde $(0.196 \mathrm{~g}, 1.00 \mathrm{mmol})$, chloramine-T $(0.423$ $\mathrm{g}, \quad 1.50 \mathrm{mmol})$, and L-proline $(2.30 \mathrm{mg}, 0.02 \mathrm{mmol})$ in acetonitrile $(5 \mathrm{~mL})$ within $30 \mathrm{~min}$. Flash chromatography on silica (diethylether/pentane 1:2) delivered $0.321 \mathrm{~g}(0.88 \mathrm{mmol}, 88 \%)$ of a colorless solid. For reaction conditions, see Table $2 .-\mathrm{mp}=96{ }^{\circ} \mathrm{C} .-\mathrm{R}_{f}=0.38$ (diethylether/pentane 1:2). ${ }^{1} \mathrm{H}$ NMR $\left(400 \mathrm{MHz}, \mathrm{CDCl}_{3}\right): \delta=2.23\left(\mathrm{~s}, 3 \mathrm{H}, \mathrm{C}^{4}{ }_{\mathrm{Ts}} \mathrm{CH}_{3}\right), 6.31$ (bs, $\left.1 \mathrm{H}, \mathrm{NH}\right), 6.83$ (d, $\left.J=8.0 \mathrm{~Hz}, 2 \mathrm{H}, \mathrm{C}^{3} \mathrm{H}_{\mathrm{Ts}}\right), 6.92\left(\mathrm{~d}, J=8.4 \mathrm{~Hz}, 2 \mathrm{H}, \mathrm{C}^{2} \mathrm{H}_{\mathrm{Ts}}\right), 7.18-7.26\left(\mathrm{~m}, 10 \mathrm{H}, \mathrm{CH}_{\mathrm{Ph}}\right), 9.30$ (s, $1 \mathrm{H}, \mathrm{CHO})$ ppm. $-{ }^{13} \mathrm{C}$ NMR $\left(100 \mathrm{MHz}, \mathrm{CDCl}_{3}\right): \delta=21.4\left(+, \mathrm{C}^{4}{ }_{\mathrm{Ts}} \mathrm{CH}_{3}\right), 74.2\left(\mathrm{q}, \mathrm{CR}_{4}\right)$, $126.2\left(+, \mathrm{C}^{3^{\prime}}{ }_{\mathrm{Ts}} \mathrm{H}\right), 128.6\left(+, \mathrm{CH}_{\mathrm{Ph}}\right), 128.7\left(+, \mathrm{CH}_{\mathrm{Ph}}\right), 128.8\left(+, \mathrm{CH}_{\mathrm{Ph}}\right), 129.7\left(+, \mathrm{C}^{2}{ }_{\mathrm{Ts}} \mathrm{H}\right), 134.3$

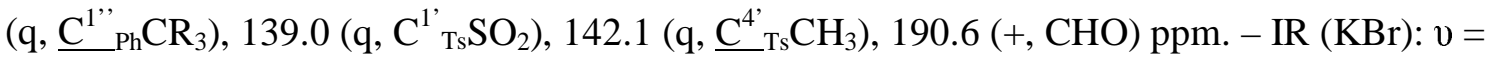




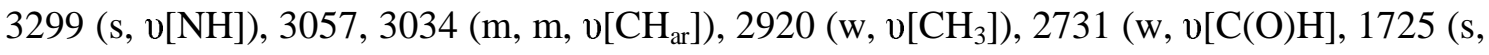
v[CO]), 1658, 1597, 1492 (w, m, m, v[C-C ar $]$ ), 1447 (m, $\left.\delta_{\text {as }}\left[\mathrm{CH}_{3}\right]\right), 1402\left(\mathrm{~m}, \delta_{\text {sy }}\left[\mathrm{CH}_{3}\right]\right), 1333$ $\left(\mathrm{s}, \mathrm{v}_{\mathrm{as}}\left[\mathrm{SO}_{2}\right], 1158\left(\mathrm{~m}, \mathrm{v}_{\mathrm{sy}}\left[\mathrm{SO}_{2}\right]\right), 852,817\left(\mathrm{~m}, \mathrm{~m}, \delta\left[\mathrm{CH}_{\mathrm{ar}}\right]\right) \mathrm{cm}^{-1}\right.$. - MS (EI, I, $\left.70 \mathrm{eV}\right)$ : $m / z(\%)=336(100)\left[\mathrm{M}^{+}-\mathrm{CHO}\right], 180(66)\left[\mathrm{M}^{+}-\mathrm{C}_{7} \mathrm{H}_{7} \mathrm{SO}_{2}\right], 155$ (57) $\left[\mathrm{C}_{7} \mathrm{H}_{7} \mathrm{SO}_{2}{ }^{+}\right], 104$ (23) $\left[\mathrm{C}_{8} \mathrm{H}_{8}{ }^{+}\right], 91(81)\left[\mathrm{C}_{7} \mathrm{H}_{7}^{+}\right], 77(29)\left[\mathrm{C}_{6} \mathrm{H}_{5}^{+}\right]$. - HRMS (I): calcd.: 336.1058 [M+CHO], found: 336.1061. - $\mathrm{C}_{21} \mathrm{H}_{19} \mathrm{NO}_{3} \mathrm{~S}$ (365.45 g/mol), calcd.: C 69.02, H 5.24, N 3.83, found: C 68.79, H 5.40, N 3.83 .

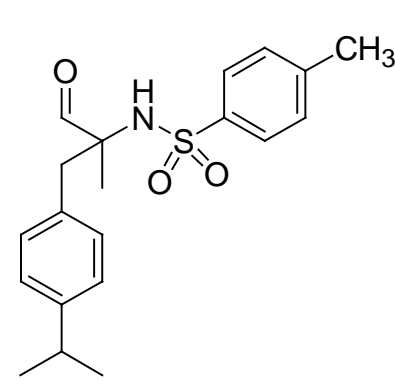

2-Methyl-3-(p-isopropylphenyl)-2-(4'"-toluene)sulfonylaminopropionaldehyde 2i: The compound was synthesized following GP 3, using 2-methyl-3-(p-isopropylphenyl)propionaldehyde $(0.226 \mathrm{~g}$, $1.00 \mathrm{mmol})$, chloramine-T (0.423 g, $1.50 \mathrm{mmol})$, and L-proline $(2.30 \mathrm{mg}, 0.02 \mathrm{mmol})$ in acetonitrile $(5 \mathrm{~mL})$ within $1 \mathrm{~d}$. Flash chromatography on silica (diethylether/pentane 1:2) delivered 0.298 $\mathrm{g}(0.83 \mathrm{mmol}, 83 \%)$ of a colorless solid. For reaction conditions, see Table $2 .-\mathrm{mp}=127{ }^{\circ} \mathrm{C}$. - $\mathrm{R}_{f}=0.40$ (diethylether/pentane 1:2). ${ }^{1}{ }^{1} \mathrm{H} \mathrm{NMR}\left(400 \mathrm{MHz}, \mathrm{CDCl}_{3}\right): \delta=1.26(\mathrm{~d}, J=6.9 \mathrm{~Hz}$, $6 \mathrm{H}, i \mathrm{Pr}$ ), 1.29 (s, $3 \mathrm{H}, \mathrm{CR}_{3} \mathrm{CH}_{3}$ ), 2.43 (s, $3 \mathrm{H}, \mathrm{C}^{4}{ }_{\mathrm{Ts}} \mathrm{CH}_{3}$ ), 2.90 (sept., $J=6.9 \mathrm{~Hz}, 1 \mathrm{H}, i \mathrm{Pr}$ ), 3.04 (s, $\left.2 \mathrm{H}, \mathrm{C}_{2} \mathrm{RCR}_{4}\right), 5.44$ (bs, $\left.1 \mathrm{H}, \mathrm{NH}\right), 7.08$ (d, $\left.J=8.1 \mathrm{~Hz}, 2 \mathrm{H}, \mathrm{C}^{3} \mathrm{H}_{\mathrm{Ts}}\right), 7.17(\mathrm{~d}, J=8.0$ $\left.\mathrm{Hz}, 2 \mathrm{H}, \mathrm{C}^{3} \mathrm{H}_{\mathrm{ar}}\right), 7.28\left(\mathrm{~d}, J=8.0 \mathrm{~Hz}, 2 \mathrm{H}, \mathrm{C}^{2} \mathrm{H}_{\mathrm{Ts}}\right), 7.75\left(\mathrm{~d}, J=8.3 \mathrm{~Hz}, 2 \mathrm{H}, \mathrm{C}^{2} \mathrm{H}_{\mathrm{ar}}\right), 9.53$ (s, $1 \mathrm{H}, \mathrm{CHO})$ ppm. $-{ }^{13} \mathrm{C} \mathrm{NMR}\left(100 \mathrm{MHz}, \mathrm{CDCl}_{3}\right): \delta=19.7\left(+, \mathrm{CR}_{3} \underline{\mathrm{CH}}_{3}\right), 21.5\left(+, \mathrm{C}^{4}{ }_{\mathrm{Ts}} \underline{\mathrm{CH}_{3}}\right)$, 24.0 (+, iPr), 33.7 (+, iPr), 42.7 (-, $\left.\mathrm{CH}_{2} \mathrm{RCR}_{4}\right), 65.7$ (q, $\left.\mathrm{CR}_{4}\right), 126.8\left(+, \mathrm{C}^{3{ }^{\prime}}{ }_{\mathrm{Ts}} \mathrm{H}\right), 126.8$ (+, $\left.\mathrm{C}^{3{ }^{\prime \prime}}{ }_{\mathrm{ar}} \mathrm{H}\right), 129.7\left(+, \mathrm{C}^{2}{ }_{\mathrm{Ts}} \mathrm{H}\right), 130.5\left(+, \mathrm{C}^{2}{ }_{\mathrm{ar}} \mathrm{H}\right), 131.1\left(\mathrm{q}, \mathrm{C}^{1 ”}{ }_{\mathrm{ar}} \mathrm{CH}_{2} \mathrm{R}\right), 139.8\left(\mathrm{q}, \mathrm{C}^{1}{ }_{\mathrm{Ts}} \mathrm{SO}_{2}\right), 143.5$

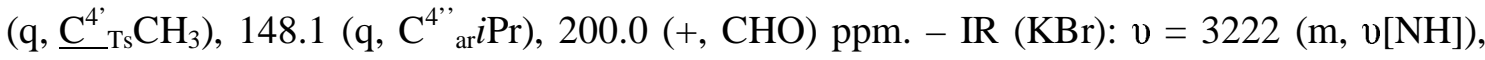
3071 (w, v[CHar $), 2962,2870$ (m, w, v[C $\left.\left.\mathrm{CH}_{3}\right]\right), 1717$ (m, v[CO]), 1598, 1513, 1498 (w, w, w, $\left.v\left[\mathrm{C}-\mathrm{C}_{\mathrm{ar}}\right]\right), 1436\left(\mathrm{~m}, \delta_{\mathrm{as}}\left[\mathrm{CH}_{3}\right]\right), 1390\left(\mathrm{w}, \delta_{\mathrm{sy}}\left[\mathrm{CH}_{3}\right]\right), 1332\left(\mathrm{~m}, \mathrm{v}_{\mathrm{as}}\left[\mathrm{SO}_{2}\right], 1170\left(\mathrm{~m}, \mathrm{v}_{\mathrm{sy}}\left[\mathrm{SO}_{2}\right]\right), 839\right.$, $814\left(\mathrm{w}, \mathrm{m}, \delta\left[\mathrm{CH}_{\mathrm{ar}}\right]\right) \mathrm{cm}^{-1}$. - MS (EI, I, $\left.70 \mathrm{eV}\right): \mathrm{m} / z(\%)=330(53)\left[\mathrm{M}^{+}-\mathrm{CHO}\right], 226$ (20) $\left[\mathrm{C}_{10} \mathrm{H}_{12} \mathrm{NO}_{3} \mathrm{~S}^{+}\right], 171$ (27) $\left[\mathrm{C}_{7} \mathrm{H}_{10} \mathrm{NO}_{2} \mathrm{~S}^{+}\right], 155(55)\left[\mathrm{C}_{7} \mathrm{H}_{7} \mathrm{SO}_{2}^{+}\right], 133$ (84) $\left[\mathrm{C}_{10} \mathrm{H}_{13}{ }^{+}\right], 91$ (100) $\left[\mathrm{C}_{7} \mathrm{H}_{7}^{+}\right], 77$ (6) $\left[\mathrm{C}_{6} \mathrm{H}_{5}^{+}\right] .-$HRMS (I): calcd.: 359.1555 [M $\left.{ }^{+}\right]$, found: 359.1560. $-\mathrm{C}_{20} \mathrm{H}_{25} \mathrm{NO}_{3} \mathrm{~S}$ (359.48 g/mol), calcd.: C 66.82, H 7.01, N 3.90, found: C 66.88, H 7.07, N 3.87. 


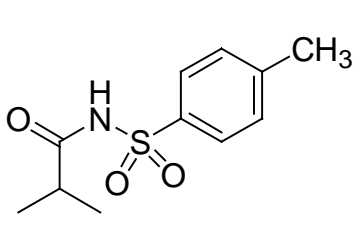

$N$-Isobutyryl-4-methylbenzenesulfonamide 4a: The compound was synthesized following GP 3, using isobutyraldehyde $(0.072 \mathrm{~g}$, $1.00 \mathrm{mmol})$, chloramine-T $(0.423 \mathrm{~g}, 1.50 \mathrm{mmol})$, and L-proline $(2.30$ $\mathrm{mg}, \quad 0.02 \mathrm{mmol})$ in acetonitrile $(5 \mathrm{~mL})$ within $1 \mathrm{~d}$. Flash chromatography on silica (diethylether/pentane 1:2) delivered $0.200 \mathrm{~g}(0.83 \mathrm{mmol}, 83 \%)$ of a colorless solid. For reaction conditions, see Table 3. $-\mathrm{mp}=124{ }^{\circ} \mathrm{C} .-\mathrm{R}_{f}=0.20$ (diethylether/pentane 1:2). $-{ }^{1} \mathrm{H}$ NMR (400 MHz, $\mathrm{CDCl}_{3}$ ): $\delta=1.03(\mathrm{~d}, J=6.9 \mathrm{~Hz}, 6 \mathrm{H}, 2 \mathrm{x}$ $\mathrm{CH}_{3}$ ), 2.37 (sept., $J=6.9 \mathrm{~Hz}, 1 \mathrm{H}, \mathrm{CHR}_{2} \mathrm{CONHR}$ ), 2.37 (s, $3 \mathrm{H}, \mathrm{C}^{4}{ }_{\mathrm{Ts}} \mathrm{CH}_{3}$ ), 7.28 (d, $\left.J=8.1 \mathrm{~Hz}, 2 \mathrm{H}, \mathrm{C}^{3} \mathrm{H}_{\mathrm{Ts}}\right), 7.88\left(\mathrm{~d}, J=8.4 \mathrm{~Hz}, 2 \mathrm{H}, \mathrm{C}^{2} \mathrm{H}_{\mathrm{Ts}}\right), 8.81$ (bs, $\left.1 \mathrm{H}, \mathrm{NH}\right) \mathrm{ppm}$. ${ }^{13} \mathrm{C}$ NMR $\left(100 \mathrm{MHz}, \mathrm{CDCl}_{3}\right): \delta=18.6\left(+, \mathrm{CH}_{3}\right), 21.7\left(+, \mathrm{C}^{4}{ }_{\mathrm{Ts}} \mathrm{CH}_{3}\right), 35.7\left(-, \mathrm{CHR}_{2} \mathrm{CONHR}\right)$, $128.3\left(+, \mathrm{C}^{3}{ }_{\mathrm{Ts}} \mathrm{H}\right), 129.7\left(+, \mathrm{C}^{2}{ }_{\mathrm{Ts}} \mathrm{H}\right), 135.5\left(\mathrm{q}, \mathrm{C}^{1}{ }_{\mathrm{Ts}} \mathrm{SO}_{2}\right), 145.1\left(\mathrm{q}, \underline{\mathrm{C}}^{4^{\prime}} \mathrm{Ts}_{\mathrm{Ts}} \mathrm{CH}_{3}\right), 174.7$ (q, $\mathrm{CH}_{2} \mathrm{RCONHR}$ ) ppm. - IR (KBr): v = $3256(\mathrm{~s}, \mathrm{v}[\mathrm{NH}]), 3054\left(\mathrm{w}, \mathrm{v}\left[\mathrm{CH}_{\mathrm{ar}}\right]\right), 2974,2930,2874$ (m, m, m, v[CH]), 1730 (s, v[CO]), 1597, 1493 (m, m, v[C-Car $]$ ), $1437\left(\mathrm{~s}, \delta_{\text {as }}\left[\mathrm{CH}_{3}\right]\right), 1328$ (s, $v_{\text {as }}\left[\mathrm{SO}_{2}\right], 1170\left(\mathrm{~s}, \mathrm{v}_{\mathrm{sy}}\left[\mathrm{SO}_{2}\right]\right), 838,812\left(\mathrm{~m}, \mathrm{~m}, \delta\left[\mathrm{CH}_{\mathrm{ar}}\right]\right) \mathrm{cm}^{-1} .-\mathrm{MS}(\mathrm{EI}, \mathrm{I}, 70 \mathrm{eV}): \mathrm{m} / z(\%)=$ 198 (1) $\left[\mathrm{M}^{+}-\mathrm{C}_{3} \mathrm{H}_{8}\right], 155$ (14) $\left[\mathrm{C}_{7} \mathrm{H}_{7} \mathrm{SO}_{2}^{+}\right], 149$ (16) $\left[\mathrm{M}^{+}-\mathrm{C}_{7} \mathrm{H}_{7} \mathrm{SO}_{2}\right], 108$ (78), 91 (15) $\left[\mathrm{C}_{7} \mathrm{H}_{7}^{+}\right]$, 41 (100) $\left[\mathrm{C}_{3} \mathrm{H}_{5}^{+}\right]$. - HRMS (I): calcd.: $241.0773\left[\mathrm{M}^{+}\right]$, found: 241.0775. - $\mathrm{C}_{11} \mathrm{H}_{15} \mathrm{NO}_{3} \mathrm{~S}$ (241.31 g/mol), calcd.: C 54.75, H 6.27, N 5.80, found: C 54.63, H 6.30, N 5.91.

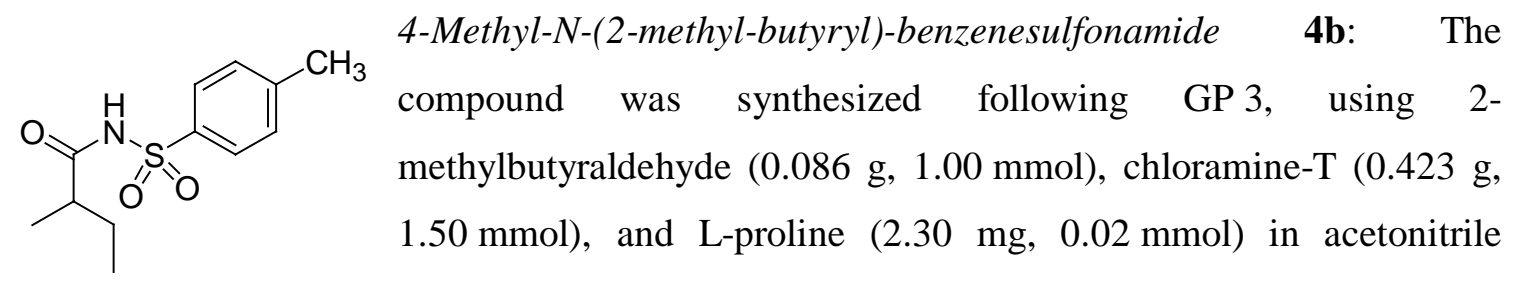
$(5 \mathrm{~mL})$ within $1 \mathrm{~d}$. Flash chromatography on silica (diethylether/pentane 1:2) delivered 0.207 $\mathrm{g}(0.81 \mathrm{mmol}, 81 \%)$ of a colorless solid. For reaction conditions, see Table $3 .-\mathrm{mp}=109{ }^{\circ} \mathrm{C}$. $-\mathrm{R}_{f}=0.27$ (diethylether/pentane 1:2). $-{ }^{1} \mathrm{H} \mathrm{NMR}\left(400 \mathrm{MHz}, \mathrm{CDCl}_{3}\right): \delta=0.72(\mathrm{t}, J=7.4 \mathrm{~Hz}$, $3 \mathrm{H}, \mathrm{CH}_{3}$ ), 1.00 (d, $J=6.9 \mathrm{~Hz}, 3 \mathrm{H}, \mathrm{CH}_{3}$ ), 1.33 (sept., $J=7.1 \mathrm{~Hz}, 1 \mathrm{H}, \mathrm{CH}_{2}$ ), 1.52 (sept., $J=$ $7.4 \mathrm{~Hz}, 1 \mathrm{H}, \mathrm{CH}_{2}$ ), 2.16 (sext., $J=6.9 \mathrm{~Hz}, 1 \mathrm{H}, \mathrm{CHR}_{2} \mathrm{CONHR}$ ), 2.38 (s, $3 \mathrm{H}, \mathrm{C}^{4}{ }_{\mathrm{Ts}} \mathrm{CH}_{3}$ ), 7.28 (d, $\left.J=8.2 \mathrm{~Hz}, 2 \mathrm{H}, \mathrm{C}^{3} \mathrm{H}_{\mathrm{Ts}}\right), 7.88$ (d, J = 8.4 Hz, $2 \mathrm{H}, \mathrm{C}^{2} \mathrm{H}_{\mathrm{Ts}}$ ), 8.75 (bs, $\left.1 \mathrm{H}, \mathrm{NH}\right) \mathrm{ppm}$. ${ }^{13} \mathrm{C}$ NMR $\left(100 \mathrm{MHz}, \mathrm{CDCl}_{3}\right): \delta=11.3\left(+, \mathrm{CH}_{3}\right), 16.3\left(+, \mathrm{CH}_{3}\right), 21.7\left(+, \mathrm{C}^{4}{ }_{\mathrm{Ts}} \underline{\mathrm{CH}_{3}}\right), 26.7(-$, $\left.\mathrm{CH}_{2}\right), 42.8$ (+, $\left.\underline{\mathrm{C} H R}{ }_{2} \mathrm{CONHR}\right), 128.4\left(+, \mathrm{C}^{3{ }^{\prime}}{ }_{\mathrm{Ts}} \mathrm{H}\right), 129.6\left(+, \mathrm{C}^{2}{ }_{\mathrm{Ts}} \mathrm{H}\right), 135.5\left(\mathrm{q}, \mathrm{C}^{1}{ }_{\mathrm{Ts}} \mathrm{SO}_{2}\right), 145.1$ (q, $\underline{\mathrm{C}}^{4}{ }_{\mathrm{Ts}} \mathrm{CH}_{3}$ ), 174.3 (q, $\mathrm{CHR}_{2}$ CONHR) ppm. - IR (KBr): v = 3253 (m, v[NH]), 3051 (w, $\left.v\left[\mathrm{CH}_{\mathrm{ar}}\right]\right), 2971,2931,2875$ (m, m, m, v[CH]), 1726 (m, $\left.\delta[\mathrm{NH}]\right), 1656$ (m, v[CO]), 1596, 1493 $\left(\mathrm{m}, \mathrm{m}, \mathrm{v}\left[\mathrm{C}-\mathrm{C}_{\mathrm{ar}}\right]\right), 1431\left(\mathrm{~m}, \delta_{\mathrm{as}}\left[\mathrm{CH}_{3}\right]\right), 1378\left(\mathrm{~m}, \delta_{\mathrm{sy}}\left[\mathrm{CH}_{3}\right]\right), 1329\left(\mathrm{~m}, \mathrm{v}_{\mathrm{as}}\left[\mathrm{SO}_{2}\right], 1166(\mathrm{~m}\right.$, 
$\left.v_{\text {sy }}\left[\mathrm{SO}_{2}\right]\right), 827,812\left(\mathrm{~m}, \mathrm{~m}, \delta\left[\mathrm{CH}_{\mathrm{ar}}\right]\right) \mathrm{cm}^{-1}$. $-\mathrm{MS}(\mathrm{EI}, \mathrm{I}, 70 \mathrm{eV}): \mathrm{m} / z(\%)=255(1)\left[\mathrm{M}^{+}\right], 155$ (50) $\left[\mathrm{C}_{7} \mathrm{H}_{7} \mathrm{SO}_{2}{ }^{+}\right], 108$ (100), 91 (73) $\left[\mathrm{C}_{7} \mathrm{H}_{7}{ }^{+}\right], 65$ (12), 57 (36) $\left[\mathrm{C}_{4} \mathrm{H}_{8}{ }^{+}\right]$. - HRMS (I): calcd.: 255.0929, found: 255.0927. $-\mathrm{C}_{12} \mathrm{H}_{17} \mathrm{NO}_{3} \mathrm{~S}$ (255.33 g/mol), calcd.: C 56.45, H 6.71, N 5.49, found: C 56.36, H 6.78, N 5.74.

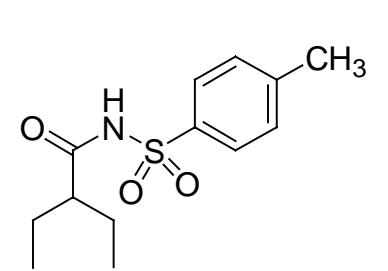

4-Methyl-N-(2-ethyl-butyryl)-benzenesulfonamide 4c: The compound was synthesized following GP 3, using 2-ethylbutyraldehyde $(0.100$ g, $1.00 \mathrm{mmol}$ chloramine-T $(0.423 \mathrm{~g}, 1.50 \mathrm{mmol})$, and L-proline $(2.30$ $\mathrm{mg}, \quad 0.02 \mathrm{mmol})$ in acetonitrile $(5 \mathrm{~mL})$ within $1 \mathrm{~d}$. Flash chromatography on silica (diethylether/pentane $1: 2)$ delivered $0.210 \mathrm{~g}(0.78 \mathrm{mmol}, 78 \%)$ of a colorless solid. For reaction conditions, see Table 3. $-\mathrm{mp}=97{ }^{\circ} \mathrm{C} .-\mathrm{R}_{f}=0.30$ (diethylether/pentane 1:2). $-{ }^{1} \mathrm{H}$ NMR $\left(400 \mathrm{MHz}, \mathrm{CDCl}_{3}\right): \delta=0.77(\mathrm{~d}, J=7.4 \mathrm{~Hz}, 6 \mathrm{H}, 2 \mathrm{x}$ $\left.\mathrm{CH}_{3}\right), 1.39-1.49\left(\mathrm{~m}, J=7.4 \mathrm{~Hz}, 2 \mathrm{H}, \mathrm{CH}_{2}\right), 1.50-1.61\left(\mathrm{~m}, J=7.4 \mathrm{~Hz}, 2 \mathrm{H}, \mathrm{CH}_{2}\right), 2.08$ (tt, $J$ = 8.7, 5.4 Hz, $\left.1 \mathrm{H}, \underline{\mathrm{CHR}}_{2} \mathrm{CONHR}\right), 2.45\left(\mathrm{~s}, 3 \mathrm{H}, \mathrm{C}^{4}{ }_{\mathrm{Ts}} \mathrm{CH}_{3}\right), 7.36\left(\mathrm{~d}, J=8.1 \mathrm{~Hz}, 2 \mathrm{H}, \mathrm{C}^{3}{ }^{\prime} \mathrm{H}_{\mathrm{Ts}}\right)$, $7.98\left(\mathrm{~d}, J=8.4 \mathrm{~Hz}, 2 \mathrm{H}, \mathrm{C}^{2} \mathrm{H}_{\mathrm{Ts}}\right), 9.24(\mathrm{bs}, 1 \mathrm{H}, \mathrm{NH}) \mathrm{ppm} .-{ }^{13} \mathrm{C} \mathrm{NMR}\left(100 \mathrm{MHz}, \mathrm{CDCl}_{3}\right): \delta=$ $11.5\left(+, 2 \mathrm{x} \mathrm{CH}_{3}\right), 21.7\left(+, \mathrm{C}^{4}{ }_{\mathrm{Ts}} \underline{\mathrm{C}}_{3}\right), 25.0$ (-, 2x CH $\left.\mathrm{CH}_{2}\right), 50.7$ (+, $\left.\underline{\mathrm{CHR}}_{2} \mathrm{CONHR}\right), 128.3$ (+,

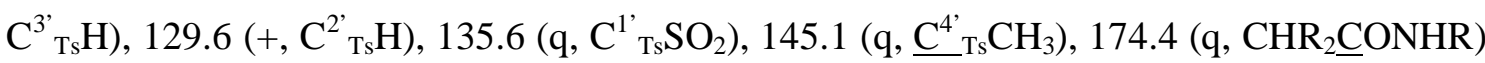
ppm. - IR (KBr): v = 3207 (m, v[NH]), 2962, 2935, 2875 (m, w, w, v[CH]), 1686 (m, v[CO]), $1597,1495\left(\mathrm{w}, \mathrm{w}, \mathrm{v}\left[\mathrm{C}-\mathrm{C}_{\mathrm{ar}}\right]\right), 1439\left(\mathrm{~m}, \delta_{\mathrm{as}}\left[\mathrm{CH}_{3}\right]\right), 1380\left(\mathrm{w}, \delta_{\mathrm{sy}}\left[\mathrm{CH}_{3}\right]\right), 1348\left(\mathrm{~m}, \mathrm{v}_{\mathrm{as}}\left[\mathrm{SO}_{2}\right], 1159\right.$ $\left(\mathrm{m}, v_{\mathrm{sy}}\left[\mathrm{SO}_{2}\right]\right), 835,811\left(\mathrm{~m}, \mathrm{~m}, \delta\left[\mathrm{CH}_{\mathrm{ar}}\right]\right) \mathrm{cm}^{-1}$. - MS (EI, I, $\left.70 \mathrm{eV}\right): \mathrm{m} / z(\%)=240(3)\left[\mathrm{M}^{+}-\right.$ $\left.\mathrm{C}_{2} \mathrm{H}_{5}\right], 205$ (7), 155 (27) $\left[\mathrm{C}_{7} \mathrm{H}_{7} \mathrm{SO}_{2}^{+}\right], 108$ (100), 91 (47) $\left[\mathrm{C}_{7} \mathrm{H}_{7}^{+}\right], 71$ (19), 65 (6), 43 (16) $\left[\mathrm{C}_{3} \mathrm{H}_{8}{ }^{+}\right]$. - HRMS (I): calcd.: 269.1086, found: 269.1084. - $\mathrm{C}_{13} \mathrm{H}_{19} \mathrm{NO}_{3} \mathrm{~S}$ (269.36 g/mol), calcd.: C 57.97, H 7.11, N 5.20, found: C 58.00, H 7.18, N 5.27.

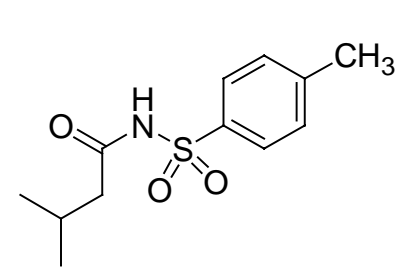

4-Methyl-N-(3-methyl-butyryl)-benzenesulfonamide $\quad \mathbf{4 d}$ : The compound was synthesized following GP 3, using 3methylbutyrladehyde (0.086 g, $1.00 \mathrm{mmol})$, chloramine-T $(0.423 \mathrm{~g}$, $1.50 \mathrm{mmol})$, and L-proline $(2.30 \mathrm{mg}, 0.02 \mathrm{mmol})$ in acetonitrile $(5 \mathrm{~mL})$ within $1 \mathrm{~d}$. Flash chromatography on silica (diethylether/pentane 1:2) delivered 0.219 $\mathrm{g}(0.86 \mathrm{mmol}, 86 \%)$ of a colorless solid. For reaction conditions, see Table $3 .-\mathrm{mp}=113{ }^{\circ} \mathrm{C}$. $-\mathrm{R}_{f}=0.25$ (diethylether/pentane 1:2). ${ }^{1} \mathrm{H}$ NMR $\left(400 \mathrm{MHz}, \mathrm{CDCl}_{3}\right): \delta=0.85(\mathrm{~d}, J=6.6 \mathrm{~Hz}$, $6 \mathrm{H}, i \operatorname{Pr}$ ), 2.05 (sept., $J=6.6 \mathrm{~Hz}, 1 \mathrm{H}, i \mathrm{Pr}), 2.14$ (d, $J=6.9 \mathrm{~Hz}, 2 \mathrm{H}, \mathrm{C}_{2} \mathrm{RCONHR}$ ), 2.43 (s, 
$\left.3 \mathrm{H}, \mathrm{C}^{4^{\prime}}{ }_{\mathrm{Ts}} \mathrm{CH}_{3}\right), 7.34\left(\mathrm{~d}, J=8.1 \mathrm{~Hz}, 2 \mathrm{H}, \mathrm{C}^{3{ }^{\prime}} \mathrm{H}_{\mathrm{Ts}}\right), 7.96\left(\mathrm{~d}, J=8.4 \mathrm{~Hz}, 2 \mathrm{H}, \mathrm{C}^{2} \mathrm{H}_{\mathrm{Ts}}\right), 9.40(\mathrm{bs}$, $1 \mathrm{H}, \mathrm{NH})$ ppm. $-{ }^{13} \mathrm{C} \mathrm{NMR}\left(100 \mathrm{MHz}, \mathrm{CDCl}_{3}\right): \delta=21.7\left(+, \mathrm{C}^{4}{ }_{\mathrm{Ts}} \mathrm{C}_{3}\right), 22.2(+, i \mathrm{Pr}), 25.6(+$, $i \mathrm{Pr}), 45.2$ (-, $\left.\mathrm{CH}_{2} \mathrm{RCONHR}\right), 128.3\left(+, \mathrm{C}^{3}{ }_{\mathrm{Ts}} \mathrm{H}\right), 129.6\left(+, \mathrm{C}^{2}{ }_{\mathrm{Ts}} \mathrm{H}\right), 135.6\left(\mathrm{q}, \mathrm{C}^{1}{ }_{\mathrm{Ts}} \mathrm{SO}_{2}\right), 145.1$ (q, $\left.\underline{\mathrm{C}}^{4}{ }_{\mathrm{Ts}} \mathrm{CH}_{3}\right), 171.1$ (q, $\mathrm{CH}_{2} \mathrm{RCONHR}$ ) ppm. - IR (KBr): v = 3264 (m, v[NH]), 3093, 3057 (w, w, v[CHar $]$ ), 2954, 2867 (m, m, v[CH]), 1719 (m, $\delta[\mathrm{NH}]), 1678$ (w, v[CO]), 1595, 1492 $\left(\mathrm{m}, \mathrm{m}, \mathrm{v}\left[\mathrm{C}-\mathrm{C}_{\mathrm{ar}}\right]\right), 1433\left(\mathrm{~m}, \delta_{\mathrm{as}}\left[\mathrm{CH}_{3}\right]\right), 1366\left(\mathrm{~m}, \delta_{\mathrm{sy}}\left[\mathrm{CH}_{3}\right]\right), 1354\left(\mathrm{~m}, \mathrm{v}_{\mathrm{as}}\left[\mathrm{SO}_{2}\right], 1151(\mathrm{~m}\right.$, $\left.v_{\mathrm{sy}}\left[\mathrm{SO}_{2}\right]\right), 854,832\left(\mathrm{~m}, \mathrm{~m}, \delta\left[\mathrm{CH}_{\mathrm{ar}}\right]\right) \mathrm{cm}^{-1}$. - MS (EI, I, $\left.70 \mathrm{eV}\right): m / z(\%)=240(1)\left[\mathrm{M}^{+}-\mathrm{CH}_{3}\right]$, 191 (7), 155 (16) $\left[\mathrm{C}_{7} \mathrm{H}_{7} \mathrm{SO}_{2}{ }^{+}\right], 108$ (100), 91 (40) $\left[\mathrm{C}_{7} \mathrm{H}_{7}{ }^{+}\right], 77$ (1) $\left[\mathrm{C}_{6} \mathrm{H}_{5}{ }^{+}\right]$. - HRMS (I): calcd.: 256.1007 $\left[\mathrm{M}^{+}+\mathrm{H}\right]$, found: $256.1004 .-\mathrm{C}_{12} \mathrm{H}_{17} \mathrm{NO}_{3} \mathrm{~S}(255.33 \mathrm{~g} / \mathrm{mol})$, calcd.: C 56.45, H 6.71, N 5.49, found: C 56.62, H 6.67, N 5.49.

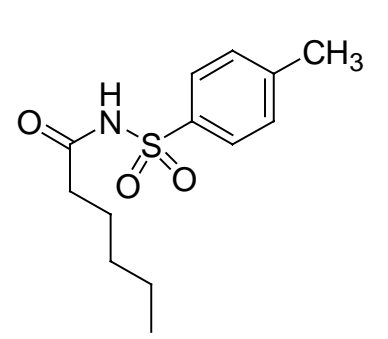

4-Methyl-N-(hexanoyl)-benzenesulfonamide 4e: The compound was synthesized following GP 3, using hexanal $(0.100 \mathrm{~g}, 1.00 \mathrm{mmol})$, chloramine-T (0.423 g, $1.50 \mathrm{mmol})$, and L-proline $(2.30 \mathrm{mg}$, $0.02 \mathrm{mmol})$ in acetonitrile $(5 \mathrm{~mL})$ within $2 \mathrm{~d}$. Flash chromatography on silica (diethylether/pentane 1:2) delivered $0.204 \mathrm{~g}(0.76 \mathrm{mmol}$, $76 \%$ ) of a colorless solid. For reaction conditions, see Table $3 .-\mathrm{mp}=66^{\circ} \mathrm{C} .-\mathrm{R}_{f}=0.34$ (diethylether/pentane 1:2). $-{ }^{1} \mathrm{H} \mathrm{NMR}\left(400 \mathrm{MHz}, \mathrm{CDCl}_{3}\right): \delta=0.84\left(\mathrm{t}, J=7.0 \mathrm{~Hz}, 3 \mathrm{H}, \mathrm{CH}_{3}\right.$ ), $1.16-1.30$ (m, $4 \mathrm{H}, 2 \mathrm{x} \mathrm{CH}$ ), 1.56 (quint., $J=7.5 \mathrm{~Hz}, 2 \mathrm{H}, \mathrm{CH}_{2}$ ), $2.26(\mathrm{t}, J=7.5 \mathrm{~Hz}, 2 \mathrm{H}$, $\mathrm{C}_{2} \mathrm{RCONHR}$ ), 2.46 (s, $\left.3 \mathrm{H}, \mathrm{C}^{4}{ }_{\mathrm{Ts}} \mathrm{CH}_{3}\right), 7.36\left(\mathrm{~d}, J=8.5 \mathrm{~Hz}, 2 \mathrm{H}, \mathrm{C}^{3}{ }^{\prime} \mathrm{H}_{\mathrm{Ts}}\right), 7.97$ (d, J=8.4 Hz, $2 \mathrm{H}, \mathrm{C}^{2} \mathrm{H}_{\mathrm{Ts}}$ ), 9.02 (bs, $\left.1 \mathrm{H}, \mathrm{NH}\right)$ ppm. $-{ }^{13} \mathrm{C} \mathrm{NMR}\left(100 \mathrm{MHz}, \mathrm{CDCl}_{3}\right): \delta=13.8\left(+, \mathrm{CH}_{3}\right), 21.7$ $\left(+, \mathrm{C}^{4}{ }_{\mathrm{Ts}} \underline{\mathrm{CH}}_{3}\right), 22.2$ (-, $\left.\mathrm{CH}_{2}\right), 24.0\left(-, \mathrm{CH}_{2}\right), 31.0\left(-, \mathrm{CH}_{2}\right), 36.2$ (-, $\left.\mathrm{CH}_{2} \mathrm{RCONHR}\right), 128.3$ (+,

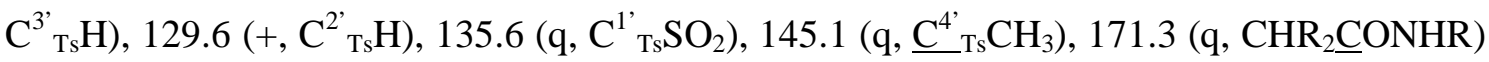
ppm. - IR (KBr): v = 3068 (m, v[NH]), 2961, 2937, 2873 (m, m, m, v[CH]), 1678 (m, v[CO]), $1597\left(\mathrm{~m}, \mathrm{v}\left[\mathrm{C}-\mathrm{C}_{\mathrm{ar}}\right]\right), 1457\left(\mathrm{~m}, \delta_{\mathrm{as}}\left[\mathrm{CH}_{3}\right]\right), 1380\left(\mathrm{w}, \delta_{\mathrm{sy}}\left[\mathrm{CH}_{3}\right]\right), 1343\left(\mathrm{~m}, \mathrm{v}_{\mathrm{as}}\left[\mathrm{SO}_{2}\right], 1155\right.$ $\left(\mathrm{m}, \mathrm{v}_{\mathrm{sy}}\left[\mathrm{SO}_{2}\right]\right), 841,819\left(\mathrm{~m}, \mathrm{~m}, \delta\left[\mathrm{CH}_{\mathrm{ar}}\right]\right) \mathrm{cm}^{-1}$. - MS (EI, I, $\left.70 \mathrm{eV}\right): \mathrm{m} / z(\%)=213(4), 205(8)$, 155 (27) $\left[\mathrm{C}_{7} \mathrm{H}_{7} \mathrm{SO}_{2}^{+}\right], 108$ (100), 91 (4) $\left[\mathrm{C}_{7} \mathrm{H}_{7}^{+}\right]$. - HRMS (I): calcd.: $270.1164\left[\mathrm{M}^{+}+\mathrm{H}\right]$, found: 270.1162. $-\mathrm{C}_{13} \mathrm{H}_{19} \mathrm{NO}_{3} \mathrm{~S}(269.36 \mathrm{~g} / \mathrm{mol})$, calcd.: C 57.97, H 7.11, N 5.20, found: C 57.83, H 7.06, N 5.27.

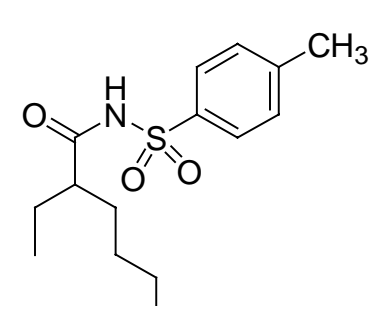

4-Methyl-N-(2-ethylhexanoyl)-benzenesulfonamide $\mathbf{4 f}$ : The compound was synthesized following GP 3, using 2-ethylhexanal $(0.128 \mathrm{~g}$, 
$1.00 \mathrm{mmol})$, chloramine-T (0.423 g, $1.50 \mathrm{mmol})$, and L-proline $(2.30 \mathrm{mg}, 0.02 \mathrm{mmol})$ in acetonitrile $(5 \mathrm{~mL})$ within $2 \mathrm{~d}$. Flash chromatography on silica (diethylether/pentane 1:2) delivered $0.214 \mathrm{~g}(0.72 \mathrm{mmol}, 72 \%)$ of a colorless solid. For reaction conditions, see Table 3. $-\mathrm{mp}=47^{\circ} \mathrm{C} .-\mathrm{R}_{f}=0.37$ (diethylether/pentane 1:2). $-{ }^{1} \mathrm{H}$ NMR $\left(400 \mathrm{MHz}, \mathrm{CDCl}_{3}\right): \delta=$ $0.77\left(\mathrm{~d}, J=7.3 \mathrm{~Hz}, 3 \mathrm{H}, \mathrm{CH}_{3}\right), 0.79\left(\mathrm{~d}, J=7.1 \mathrm{~Hz}, 3 \mathrm{H}, \mathrm{CH}_{3}\right), 0.99-1.24(\mathrm{~m}, 4 \mathrm{H}, 2 \mathrm{x} \mathrm{CH})_{2}$, $1.33-1.61$ (m, $\left.4 \mathrm{H}, 2 \mathrm{x} \mathrm{CH}_{2}\right), 2.11$ (tt, $\left.J=8.9,5.4 \mathrm{~Hz}, 1 \mathrm{H}, \mathrm{CHR}_{2} \mathrm{CONHR}\right), 2.46$ (s, $3 \mathrm{H}$, $\left.\mathrm{C}^{4}{ }_{\mathrm{Ts}} \mathrm{CH}_{3}\right), 7.36\left(\mathrm{~d}, J=8.0 \mathrm{~Hz}, 2 \mathrm{H}, \mathrm{C}^{3} \mathrm{H}_{\mathrm{Ts}}\right), 7.97\left(\mathrm{~d}, J=8.4 \mathrm{~Hz}, 2 \mathrm{H}, \mathrm{C}^{2} \mathrm{H}_{\mathrm{Ts}}\right), 9.10(\mathrm{bs}, 1 \mathrm{H}$, $\mathrm{NH})$ ppm. $-{ }^{13} \mathrm{C} \mathrm{NMR}\left(100 \mathrm{MHz}, \mathrm{CDCl}_{3}\right): \delta=11.6\left(+, \mathrm{CH}_{3}\right), 13.8\left(+, \mathrm{CH}_{3}\right), 21.7(+$,

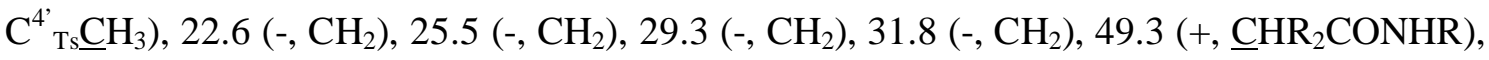
$128.3\left(+, \mathrm{C}^{3}{ }_{\mathrm{Ts}} \mathrm{H}\right), 129.6\left(+, \mathrm{C}^{2}{ }_{\mathrm{Ts}} \mathrm{H}\right), 135.6\left(\mathrm{q}, \mathrm{C}^{1}{ }^{\prime}{ }_{\mathrm{Ts}} \mathrm{SO}_{2}\right), 145.1$ (q, $\left.\mathrm{C}^{4}{ }^{\prime} \mathrm{Ts}_{\mathrm{S}} \mathrm{CH}_{3}\right), 174.4$ (q, $\mathrm{CHR}_{2}$ CONHR) ppm. - IR (KBr): v = 3237 (m, v[NH]), 2948, 2933, 2869 (m, m, m, v[CH]), $1690(\mathrm{~m}, \mathrm{v}[\mathrm{CO}]), 1598\left(\mathrm{~m}, \mathrm{v}\left[\mathrm{C}-\mathrm{C}_{\mathrm{ar}}\right]\right), 1439\left(\mathrm{~m}, \delta_{\mathrm{as}}\left[\mathrm{CH}_{3}\right]\right), 1396\left(\mathrm{~m}, \delta_{\mathrm{sy}}\left[\mathrm{CH}_{3}\right]\right), 1350(\mathrm{~m}$, $v_{\text {as }}\left[\mathrm{SO}_{2}\right], 1154\left(\mathrm{~m}, \mathrm{v}_{\mathrm{sy}}\left[\mathrm{SO}_{2}\right]\right), 831,808\left(\mathrm{~m}, \mathrm{~m}, \delta\left[\mathrm{CH}_{\mathrm{ar}}\right]\right) \mathrm{cm}^{-1}$. $-\mathrm{MS}(\mathrm{EI}, \mathrm{I}, 70 \mathrm{eV}): \mathrm{m} / z(\%)=$ 289 (7) $\left[\mathrm{M}^{+}+\mathrm{H}\right], 226(10)\left[\mathrm{C}_{10} \mathrm{H}_{12} \mathrm{NO}_{3} \mathrm{~S}^{+}\right], 177$ (7), 162 (10), 155 (33) $\left[\mathrm{C}_{7} \mathrm{H}_{7} \mathrm{SO}_{2}^{+}\right], 108$ (100), 91 (9) $\left[\mathrm{C}_{7} \mathrm{H}_{7}^{+}\right]$. - HRMS (I): calcd.: $289.1477\left[\mathrm{M}^{+}+\mathrm{H}\right]$, found: 289.1479. $-\mathrm{C}_{15} \mathrm{H}_{23} \mathrm{NO}_{3} \mathrm{~S}$ (297.41 g/mol), calcd.: C 60.58, H 7.79, N 4.71, found: C 60.48, H 7.76, N 4.79.

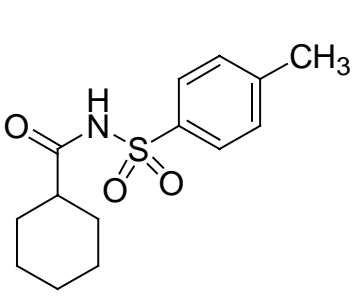

$N$-Cyclohexanecarbonyl-4-methyl-benzenesulfonamide $\quad \mathbf{4 a}: \quad$ The compound was synthesized following GP 3, using cyclohexanecarbaldehyde $(0.112 \mathrm{~g}, 1.00 \mathrm{mmol})$, chloramine- $\mathrm{T}(0.423$ $\mathrm{g}, 1.50 \mathrm{mmol})$, and L-proline $(2.30 \mathrm{mg}, 0.02 \mathrm{mmol})$ in acetonitrile $(5 \mathrm{~mL})$ within 2 d. Flash chromatography on silica (diethylether/pentane 1:2) delivered $0.242 \mathrm{~g}(0.86 \mathrm{mmol}, 86 \%)$ of a colorless solid. For reaction conditions, see Table 3. $-\mathrm{mp}=105{ }^{\circ} \mathrm{C} .-\mathrm{R}_{f}=0.35$ (diethylether/pentane 1:2). ${ }^{1} \mathrm{H}$ NMR (400 MHz, $\mathrm{CDCl}_{3}$ ): $\delta=1.05-2.21$ (m, $11 \mathrm{H}$, cyHex), 2.37 (s, $3 \mathrm{H}, \mathrm{C}^{4}{ }_{\mathrm{Ts}} \mathrm{CH}_{3}$ ), 7.27 $\left(\mathrm{d}, J=8.0 \mathrm{~Hz}, 2 \mathrm{H}, \mathrm{C}^{3} \mathrm{H}_{\mathrm{Ts}}\right), 7.87$ (d, $\left.J=8.4 \mathrm{~Hz}, 2 \mathrm{H}, \mathrm{C}^{2} \mathrm{H}_{\mathrm{Ts}}\right), 8.59$ (bs, $\left.1 \mathrm{H}, \mathrm{NH}\right) \mathrm{ppm}$. ${ }^{13} \mathrm{C}$ NMR $\left(100 \mathrm{MHz}, \mathrm{CDCl}_{3}\right): \delta=21.7\left(+, \mathrm{C}^{4}{ }_{\mathrm{Ts}} \underline{\mathrm{CH}}_{3}\right), 25.2\left(-, \mathrm{CH}_{2}\right), 25.4\left(-, \mathrm{CH}_{2}\right), 28.7(-$, $\left.\mathrm{CH}_{2}\right), 45.1$ (+, $\left.\underline{\mathrm{C} H R}{ }_{2} \mathrm{CONHR}\right), 128.3\left(+, \mathrm{C}^{3}{ }_{\mathrm{Ts}} \mathrm{H}\right), 129.6\left(+, \mathrm{C}^{2}{ }_{\mathrm{Ts}} \mathrm{H}\right), 135.6\left(\mathrm{q}, \mathrm{C}^{1}{ }_{\mathrm{Ts}} \mathrm{SO}_{2}\right), 145.1$ (q, $\left.\underline{\mathrm{C}}^{4}{ }_{T \mathrm{~s}} \mathrm{CH}_{3}\right), 173.6$ (q, $\left.\mathrm{CHR}_{2} \underline{\mathrm{CONHR}}\right) \mathrm{ppm}$. - IR (KBr): v = 3252 (m, v[NH]), 3054 (w, v[ $\left.\left[\mathrm{CH}_{\mathrm{ar}}\right]\right), 2933,2850$ (m, m, v[CH]), 1684 (w, v[CO]), 1596, 1493 (w, w, v[C-C $\mathrm{Cr}$ ), 1437 (m, $\left.\delta_{\text {as }}\left[\mathrm{CH}_{3}\right]\right), 1381\left(\mathrm{w}, \delta_{\mathrm{sy}}\left[\mathrm{CH}_{3}\right]\right), 1326\left(\mathrm{~m}, \mathrm{v}_{\mathrm{as}}\left[\mathrm{SO}_{2}\right], 1148\left(\mathrm{~m}, \mathrm{v}_{\mathrm{sy}}\left[\mathrm{SO}_{2}\right]\right), 845,814(\mathrm{~m}, \mathrm{~m}\right.$, $\left.\delta\left[\mathrm{CH}_{\mathrm{ar}}\right]\right) \mathrm{cm}^{-1}$. - MS (EI, I, $\left.70 \mathrm{eV}\right): m / z(\%)=282(19)\left[\mathrm{M}^{+}+\mathrm{H}\right], 217$ (10), 155 (7) $\left[\mathrm{C}_{7} \mathrm{H}_{7} \mathrm{SO}_{2}^{+}\right], 126(16)\left[\mathrm{M}^{+}-\mathrm{C}_{7} \mathrm{H}_{7} \mathrm{SO}_{2}\right], 108(100)\left[\mathrm{C}_{8} \mathrm{H}_{8}{ }^{+}\right], 91$ (3) $\left[\mathrm{C}_{7} \mathrm{H}_{7}{ }^{+}\right]$. - HRMS (I): calcd.: 
282.1164 [M+ $\left.\mathrm{M}^{+}+\mathrm{H}\right]$, found: 282.1166. $-\mathrm{C}_{14} \mathrm{H}_{19} \mathrm{NO}_{3} \mathrm{~S}(281.37 \mathrm{~g} / \mathrm{mol})$, calcd.: C 59.76, H 6.81, N 4.98, found: C 69.59, H 6.87, N 5.06.

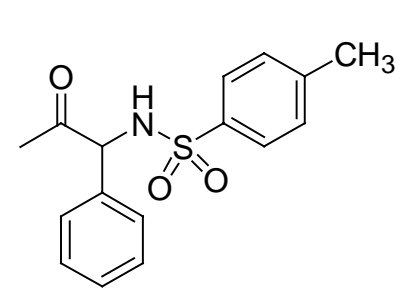

2-Phenyl-2-(4'-toluene)sulfonylaminopropanone 6a: The compound was synthesized following GP 3, using 2-phenylpropanone $(0.134$ g, $1.00 \mathrm{mmol})$, chloramine-T (0.423 g, $1.50 \mathrm{mmol})$, and L-proline $(2.30 \mathrm{mg}, 0.02 \mathrm{mmol})$ in acetonitrile $(5 \mathrm{~mL})$ within $1 \mathrm{~d}$. Flash chromatography on silica (diethylether/pentane 1:2) delivered 0.251 $\mathrm{g}(0.83 \mathrm{mmol}, 83 \%)$ of a colorless solid. For reaction conditions, see Table $3 .-\mathrm{mp}=141{ }^{\circ} \mathrm{C}$. - $\mathrm{R}_{f}=0.38$ (diethylether/pentane 1:2). $-{ }^{1} \mathrm{H} \mathrm{NMR}\left(400 \mathrm{MHz}, \mathrm{CDCl}_{3}\right): \delta=1.92(\mathrm{~s}, 3 \mathrm{H}$, $\left.\mathrm{CORCH}_{3}\right), 2.27\left(\mathrm{~s}, 3 \mathrm{H}, \mathrm{C}^{4}{ }_{\mathrm{Ts}} \mathrm{CH}_{3}\right), 5.96\left(\mathrm{~d}, J=5.0 \mathrm{~Hz}, 1 \mathrm{H}, \mathrm{CHR}_{2} \mathrm{NHR}\right), 6.00(\mathrm{~d}, J=5.0 \mathrm{~Hz}$, $1 \mathrm{H}, \mathrm{NH}), 7.02\left(\mathrm{~d}, J=8.0 \mathrm{~Hz}, 2 \mathrm{H}, \mathrm{C}^{3} \mathrm{H}_{\mathrm{Ts}}\right), 7.04\left(\mathrm{dd}, J=7.7,1.6 \mathrm{~Hz}, 2 \mathrm{H}, \mathrm{CH}_{\mathrm{Ph}}\right), 7.13-7.18$ $\left(\mathrm{m}, 3 \mathrm{H}, \mathrm{CH}_{\mathrm{Ph}}\right), 7.40\left(\mathrm{~d}, J=8.3 \mathrm{~Hz}, 2 \mathrm{H}, \mathrm{C}^{2} \mathrm{H}_{\mathrm{Ts}}\right)$ ppm. $-{ }^{13} \mathrm{C} \mathrm{NMR}\left(100 \mathrm{MHz}, \mathrm{CDCl}_{3}\right): \delta=$ $21.5\left(+, \mathrm{C}^{4}{ }_{\mathrm{Ts}} \underline{\mathrm{CH}}_{3}\right), 26.6\left(+, \mathrm{CORC}_{3}\right), 66.4\left(+, \mathrm{CHR}_{3}\right), 127.0\left(+, \mathrm{C}^{3}{ }_{\mathrm{Ts}} \mathrm{H}\right), 128.1\left(+, \mathrm{CH}_{\mathrm{Ph}}\right)$, $128.7\left(+, \mathrm{C}^{4}{ }_{\mathrm{Ph}} \mathrm{H}\right), 129.1\left(+, \mathrm{CH}_{\mathrm{Ph}}\right), 129.3\left(+, \mathrm{C}^{2}{ }_{\mathrm{Ts}} \mathrm{H}\right), 135.0\left(\mathrm{q}, \mathrm{C}^{1}{ }^{\prime}{ }_{\mathrm{Ph}} \mathrm{CHR}_{3}\right), 137.3$ (q, $\left.\mathrm{C}^{1}{ }^{1} \mathrm{Ts}_{2}\right), 143.2\left(\mathrm{q}, \underline{\mathrm{C}^{4}}{ }_{\mathrm{Ts}} \mathrm{CH}_{3}\right), 201.8\left(\mathrm{q}, \underline{\mathrm{CORCH}_{3}}\right)$ ppm. $-\mathrm{IR}(\mathrm{KBr}): v=3267(\mathrm{~m}, \mathrm{v}[\mathrm{NH}])$, 3087, $3066\left(\mathrm{w}, \mathrm{m}, \mathrm{v}\left[\mathrm{CH}_{\mathrm{ar}}\right]\right), 2959\left(\mathrm{~m}, \mathrm{v}\left[\mathrm{CH}_{3}\right]\right), 1719(\mathrm{~m}, \mathrm{v}[\mathrm{CO}]), 1598,1496(\mathrm{~m}, \mathrm{~m}, \mathrm{v}[\mathrm{C}-$ $\left.\left.\mathrm{C}_{\mathrm{ar}}\right]\right), 1438\left(\mathrm{~m}, \delta_{\mathrm{as}}\left[\mathrm{CH}_{3}\right]\right), 1341\left(\mathrm{~m}, \delta_{\mathrm{sy}}\left[\mathrm{CH}_{3}\right]\right), 1307\left(\mathrm{~m}, \mathrm{v}_{\mathrm{as}}\left[\mathrm{SO}_{2}\right], 1165\left(\mathrm{~m}, \mathrm{v}_{\mathrm{sy}}\left[\mathrm{SO}_{2}\right]\right), 818,745\right.$ $\left(\mathrm{m}, \mathrm{m}, \delta\left[\mathrm{CH}_{\mathrm{ar}}\right]\right) \mathrm{cm}^{-1}$. - MS (EI, I, $\left.70 \mathrm{eV}\right): \mathrm{m} / z(\%)=260(100)\left[\mathrm{M}^{+}-\mathrm{C}_{2} \mathrm{H}_{3} \mathrm{O}\right], 155$ (34) $\left[\mathrm{C}_{7} \mathrm{H}_{7} \mathrm{SO}_{2}^{+}\right], 104$ (7) $\left[\mathrm{C}_{8} \mathrm{H}_{8}{ }^{+}\right], 91$ (41) $\left[\mathrm{C}_{7} \mathrm{H}_{7}{ }^{+}\right], 77$ (4) $\left[\mathrm{C}_{6} \mathrm{H}_{5}^{+}\right] .-$HRMS (I): calcd.: 260.0745 $\left[\mathrm{M}^{+}-\mathrm{C}_{2} \mathrm{H}_{3} \mathrm{O}\right]$, found: 260.0740. $-\mathrm{C}_{16} \mathrm{H}_{17} \mathrm{NO}_{3} \mathrm{~S}$ (303.38 g/mol), calcd.: C 63.35, H 5.65, N 4.62, found: C 63.08, H 5.64, N 4.69.

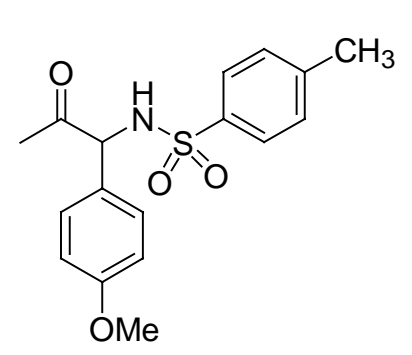

2-(4'-Methoxyphenyl-2-(4')-toluene)sulfonylaminopropanone $\mathbf{6 b}$ The compound was synthesized following GP 1, using 2-(4'methoxyphenyl)propanone $(0.164 \mathrm{~g}, 1.00 \mathrm{mmol})$, chloramine-T $(0.423 \mathrm{~g}, 1.50 \mathrm{mmol})$, and L-proline $(2.30 \mathrm{mg}, 0.02 \mathrm{mmol})$ in acetonitrile $(5 \mathrm{~mL})$ within $1 \mathrm{~d}$. Flash chromatography on silica (diethylether/pentane 1:2) delivered $0.236 \mathrm{~g}(0.71 \mathrm{mmol}, 71 \%)$ of a colorless solid. For reaction conditions and yields, see Tables $1-4 .-\mathrm{mp}=126^{\circ} \mathrm{C} .-\mathrm{R}_{f}=0.34$ (diethylether/pentane 1:2). $-{ }^{1} \mathrm{H}$ NMR (400 MHz, DMSO): $\delta=2.11$ (s, $3 \mathrm{H}, \mathrm{CORCH}_{3}$ ), 2.37 (s, $\left.3 \mathrm{H}, \mathrm{C}^{4}{ }^{\prime \prime} \mathrm{Ts}_{3} \mathrm{CH}_{3}\right), 3.49$ (bs, $\left.1 \mathrm{H}, \mathrm{NH}\right), 3.68$ (s, $\mathrm{C}^{4}{ }_{\text {ar }} \mathrm{OCH}_{3}$ ), 3.74 (s, $\left.1 \mathrm{H}, \mathrm{CHR}_{2} \mathrm{NHR}\right), 6.90$ (d, 
$\left.J=8.7 \mathrm{~Hz}, 2 \mathrm{H}, \mathrm{C}^{3 \prime} \mathrm{H}_{\mathrm{ar}}\right), 7.13\left(\mathrm{~d}, J=8.7 \mathrm{~Hz}, 2 \mathrm{H}, \mathrm{C}^{2} \mathrm{H}_{\mathrm{ar}}\right), 7.37\left(\mathrm{~d}, J=8.5 \mathrm{~Hz}, 2 \mathrm{H}, \mathrm{C}^{3{ }^{\prime \prime}} \mathrm{H}_{\mathrm{Ts}}\right)$, $7.79\left(\mathrm{~d}, J=8.2 \mathrm{~Hz}, 2 \mathrm{H}, \mathrm{C}^{2}{ }^{\prime \prime} \mathrm{H}_{\mathrm{Ts}}\right)$ ppm. $-{ }^{13} \mathrm{C} \mathrm{NMR}(100 \mathrm{MHz}, \mathrm{DMSO}): \delta=20.8(+$, $\left.\mathrm{C}^{4}{ }^{\prime \prime}{ }_{\mathrm{Ts}} \mathrm{CH}_{3}\right), 29.1\left(+, \mathrm{CORCH}_{3}\right), 48.8\left(+, \mathrm{CHR}_{3}\right), 54.9\left(+, \mathrm{C}^{4}{ }_{\mathrm{ar}} \mathrm{OCH}_{3}\right), 113.7\left(+, \mathrm{C}^{3}{ }_{\mathrm{ar}} \mathrm{H}\right), 125.6$ $\left(+, \mathrm{C}^{3{ }^{\prime \prime}}{ }_{\mathrm{Ts}} \mathrm{H}\right), 126.7\left(+, \mathrm{C}^{1}{ }_{a r} \mathrm{CHR}_{3}\right), 129.3\left(+, \mathrm{C}^{2}{ }_{\mathrm{Ts}} \mathrm{H}\right), 130.5\left(+, \mathrm{C}^{2}{ }_{\mathrm{ar}} \mathrm{H}\right), 141.4\left(\mathrm{q}, \mathrm{C}^{1{ }^{\prime \prime}} \mathrm{Ts}_{\mathrm{S}} \mathrm{SO}_{2}\right)$,

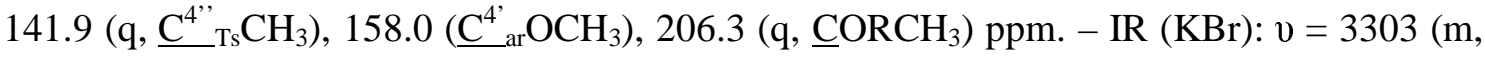
$v[\mathrm{NH}]), 3016\left(\mathrm{w}, \mathrm{v}\left[\mathrm{CH}_{\mathrm{ar}}\right]\right), 2963\left(\mathrm{w}, \mathrm{v}\left[\mathrm{CH}_{3}\right]\right), 2840\left(\mathrm{w}, \mathrm{v}\left[\mathrm{OCH}_{3}\right], 1718(\mathrm{~m}, \mathrm{v}[\mathrm{CO}]), 1586\right.$, $1490\left(\mathrm{w}, \mathrm{w}, \mathrm{v}\left[\mathrm{C}-\mathrm{C}_{\mathrm{ar}}\right]\right), 1441\left(\mathrm{w}, \delta_{\mathrm{as}}\left[\mathrm{CH}_{3}\right]\right), 1334\left(\mathrm{w}, \mathrm{v}_{\mathrm{as}}\left[\mathrm{SO}_{2}\right], 1162\left(\mathrm{~m}, \mathrm{v}_{\mathrm{sy}}\left[\mathrm{SO}_{2}\right]\right), 813(\mathrm{~m}\right.$, $\left.\delta\left[\mathrm{CH}_{\mathrm{ar}}\right]\right) \mathrm{cm}^{-1}$. - MS (EI, I, $\left.70 \mathrm{eV}\right): m / z(\%)=333(1)\left[\mathrm{M}^{+}\right], 290(100)\left[\mathrm{M}-\mathrm{C}_{2} \mathrm{H}_{3} \mathrm{O}\right], 155(27)$ $\left[\mathrm{C}_{7} \mathrm{H}_{7} \mathrm{SO}_{2}^{+}\right], 134$ (7), 104 (1) $\left[\mathrm{C}_{8} \mathrm{H}_{8}{ }^{+}\right], 91$ (39) $\left[\mathrm{C}_{7} \mathrm{H}_{7}^{+}\right], 77$ (2) $\left[\mathrm{C}_{6} \mathrm{H}_{5}^{+}\right]$. - HRMS (I): calcd.: 333.1035, found: 333.1038. $-\mathrm{C}_{17} \mathrm{H}_{19} \mathrm{NO}_{4} \mathrm{~S}$ (333.40 g/mol), calcd.: C 61.24, H 5.74, N 4.20, found: C 61.12, H 5.77, N 4.13.

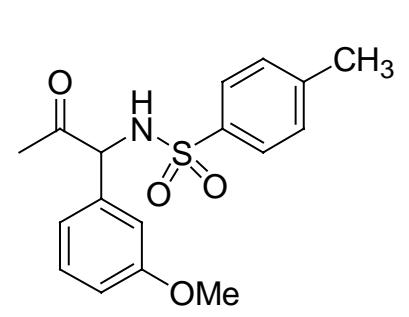

2-(3'-Methoxyphenyl)-2-(4',-toluene)sulfonylaminopropanone $\mathbf{6 c}$ The compound was synthesized following GP 3, using 2-(3'methoxyphenyl)propanone $(0.164 \mathrm{~g}, 1.00 \mathrm{mmol})$, chloramine-T $(0.423 \mathrm{~g}, 1.50 \mathrm{mmol})$, and L-proline $(2.30 \mathrm{mg}, 0.02 \mathrm{mmol})$ in acetonitrile $(5 \mathrm{~mL})$ within $1 \mathrm{~d}$. Flash chromatography on silica (diethylether/pentane 1:2) delivered $0.246 \mathrm{~g}(0.74 \mathrm{mmol}, 74 \%)$ of a colorless solid. For reaction conditions, see Table 3. $-\mathrm{mp}=123{ }^{\circ} \mathrm{C} .-\mathrm{R}_{f}=0.35$ (diethylether/pentane 1:2). ${ }^{1} \mathrm{H}$ NMR (400 MHz, DMSO): $\delta=2.13$ (s, $3 \mathrm{H}, \mathrm{CORCH}_{3}$ ), 2.37 (s, $3 \mathrm{H}, \mathrm{C}^{4}{ }_{\mathrm{Ts}} \mathrm{CH}_{3}$ ), 3.49 (bs, $1 \mathrm{H}, \mathrm{NH}), 3.73\left(\mathrm{~s}, \mathrm{C}^{3}{ }_{\mathrm{ar}} \mathrm{OCH}_{3}\right), 3.74\left(\mathrm{~s}, 1 \mathrm{H}, \mathrm{CHR}_{2} \mathrm{NHR}\right), 6.78-6.85\left(\mathrm{~m}, 2 \mathrm{H}, \mathrm{CH}_{\mathrm{ar}}\right), 7.24$ (t, $J$ $\left.=7.8 \mathrm{~Hz}, 1 \mathrm{H}, \mathrm{C}^{5} \mathrm{H}_{\mathrm{ar}}\right), 7.35\left(\mathrm{~s}, 1 \mathrm{H}, \mathrm{CH}_{\mathrm{ar}}\right), 7.37\left(\mathrm{~d}, J=8.1 \mathrm{~Hz}, 2 \mathrm{H}, \mathrm{C}^{3 ’} \mathrm{H}_{\mathrm{Ts}}\right), 7.78(\mathrm{~d}$, $\left.J=8.2 \mathrm{~Hz}, 2 \mathrm{H}, \mathrm{C}^{2}{ }^{\prime \prime} \mathrm{H}_{\mathrm{Ts}}\right)$ ppm. $-{ }^{13} \mathrm{C} \mathrm{NMR}(100 \mathrm{MHz}, \mathrm{DMSO}): \delta=20.8\left(+, \mathrm{C}^{4}{ }^{\prime \prime} \underline{\mathrm{C}}_{3}\right), 29.3$ $\left(+, \mathrm{CORCH}_{3}\right), 49.7\left(+, \mathrm{CHR}_{3}\right), 54.9\left(\mathrm{C}^{3}{ }^{\prime}{ }_{\mathrm{rOC}} \mathrm{OCH}_{3}\right), 111.9\left(+, \mathrm{C}^{4}{ }_{\text {ar }} \mathrm{H}\right), 115.2\left(+, \mathrm{C}^{2}{ }^{\prime}{ }_{\mathrm{r}} \mathrm{H}\right), 121.8$

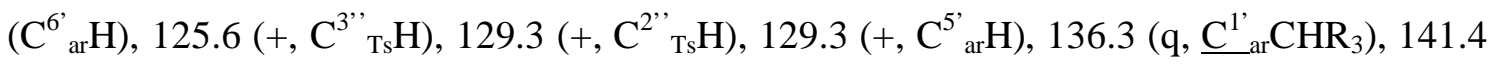

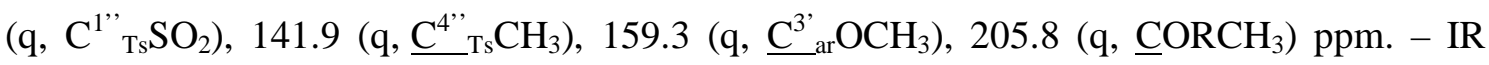
$(\mathrm{KBr}): \mathrm{v}=3289(\mathrm{~m}, \mathrm{v}[\mathrm{NH}]), 3016\left(\mathrm{~m}, \mathrm{v}\left[\mathrm{CH}_{\mathrm{ar}}\right]\right), 2966\left(\mathrm{~m}, \mathrm{v}\left[\mathrm{CH}_{3}\right]\right), 2840\left(\mathrm{w}, \mathrm{v}\left[\mathrm{OCH}_{3}\right], 1722\right.$ $(\mathrm{m}, \mathrm{v}[\mathrm{CO}]), 1587,1490\left(\mathrm{~m}, \mathrm{~m}, \mathrm{v}\left[\mathrm{C}-\mathrm{C}_{\mathrm{ar}}\right]\right), 1440\left(\mathrm{~m}, \delta_{\mathrm{as}}\left[\mathrm{CH}_{3}\right]\right), 1332\left(\mathrm{~m}, \mathrm{v}_{\mathrm{as}}\left[\mathrm{SO}_{2}\right], 1162(\mathrm{~m}\right.$, $\left.v_{\text {sy }}\left[\mathrm{SO}_{2}\right]\right), 813,781,702\left(\mathrm{~m}, \mathrm{~m}, \mathrm{~m}, \delta\left[\mathrm{CH}_{\mathrm{ar}}\right]\right) \mathrm{cm}^{-1}$. - MS (EI, I, $\left.70 \mathrm{eV}\right): \mathrm{m} / z(\%)=333(1)$ $\left[\mathrm{M}^{+}\right], 290$ (100) $\left[\mathrm{M}-\mathrm{C}_{2} \mathrm{H}_{3} \mathrm{O}\right], 174$ (16), 155 (43) $\left[\mathrm{C}_{7} \mathrm{H}_{7} \mathrm{SO}_{2}^{+}\right], 139$ (43), 104 (2) $\left[\mathrm{C}_{8} \mathrm{H}_{8}^{+}\right], 91$ (99) $\left[\mathrm{C}_{7} \mathrm{H}_{7}{ }^{+}\right], 77$ (6) $\left[\mathrm{C}_{6} \mathrm{H}_{5}{ }^{+}\right]$. - HRMS (I): calcd.: 333.1035, found: 333.1034. $-\mathrm{C}_{17} \mathrm{H}_{19} \mathrm{NO}_{4} \mathrm{~S}$ (333.40 g/mol), calcd.: C 61.24, H 5.74, N 4.20, found: C 61.33, H 5.87, N 4.17. 


\section{2-(4'-Fluorophenyl)-2-(4''-toluene)sulfonylaminopropanone $\mathbf{6 d}$}

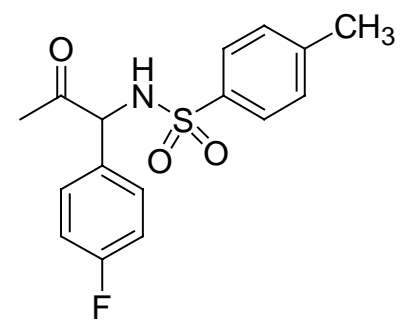

The compound was synthesized following GP 3, using 2-(4'fluorophenyl)propanone $(0.152 \mathrm{~g}, 1.00 \mathrm{mmol})$, chloramine- $\mathrm{T}(0.423$ $\mathrm{g}, 1.50 \mathrm{mmol})$, and L-proline $(2.30 \mathrm{mg}, 0.02 \mathrm{mmol})$ in acetonitrile $(5 \mathrm{~mL})$ within 2 d. Flash chromatography on silica (diethylether/pentane 1:2) delivered $0.263 \mathrm{~g}(0.82 \mathrm{mmol}, 82 \%)$ of a colorless solid. For reaction conditions, see Table 3. $-\mathrm{mp}=103{ }^{\circ} \mathrm{C} .-\mathrm{R}_{f}=0.40$ (diethylether/pentane 1:2). $-{ }^{1} \mathrm{H}$ NMR (400 MHz, DMSO): $\delta=2.14$ (s, $3 \mathrm{H}, \mathrm{CORCH}_{3}$ ), 2.36 (s, $3 \mathrm{H}, \mathrm{C}^{4}{ }_{\mathrm{Ts}} \mathrm{CH}_{3}$ ), 3.49 (bs, $\left.1 \mathrm{H}, \mathrm{NH}\right), 3.78$ (s, $\left.1 \mathrm{H}, \mathrm{CHR}_{2} \mathrm{NHR}\right), 7.13$ (t, J = 8.9 Hz, $2 \mathrm{H}$, $\left.\mathrm{CH}_{\mathrm{ar}}\right), 7.22\left(\mathrm{dd}, J=8.7,5.7 \mathrm{~Hz}, 2 \mathrm{H}, \mathrm{CH}_{\mathrm{ar}}\right), 7.36\left(\mathrm{~d}, J=7.9 \mathrm{~Hz}, 2 \mathrm{H}, \mathrm{C}^{3}{ }^{\prime \prime} \mathrm{H}_{\mathrm{Ts}}\right), 7.78(\mathrm{~d}$, $\left.J=8.3 \mathrm{~Hz}, 2 \mathrm{H}, \mathrm{C}^{2}{ }^{\prime \prime} \mathrm{H}_{\mathrm{Ts}}\right)$ ppm. $-{ }^{13} \mathrm{C} \mathrm{NMR}(100 \mathrm{MHz}, \mathrm{DMSO}): \delta=20.8\left(+, \mathrm{C}^{4}{ }_{\mathrm{Ts}}{ }^{\mathrm{C}} \mathrm{H}_{3}\right), 29.3$ $\left(+, \mathrm{CORCH}_{3}\right), 48.5\left(+, \mathrm{CHR}_{3}\right), 114.9\left(+, \mathrm{d}, J=21.1 \mathrm{~Hz}, \mathrm{C}^{3}{ }_{\mathrm{ar}} \mathrm{H}\right), 125.6\left(+, \mathrm{C}^{3{ }^{\prime \prime}}{ }_{\mathrm{Ts}} \mathrm{H}\right), 129.2(+$, $\left.\mathrm{C}^{2 ”}{ }_{\mathrm{TS}} \mathrm{H}\right), 131.0\left(\mathrm{q}, \mathrm{d}, J=3.1 \mathrm{~Hz}, \mathrm{C}^{1}{ }_{\mathrm{ar}} \mathrm{CHR}_{3}\right), 131.4\left(+, \mathrm{d}, J=8.0 \mathrm{~Hz}, \mathrm{C}^{2}{ }_{\mathrm{ar}} \mathrm{H}\right), 141.4$ (q,

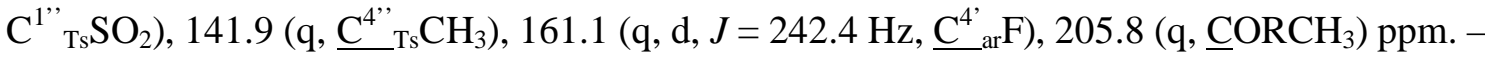
IR (KBr): v = $3311(\mathrm{~m}, \mathrm{v}[\mathrm{NH}]), 3034,3008\left(\mathrm{w}, \mathrm{w}, \mathrm{v}\left[\mathrm{CH}_{\mathrm{ar}}\right]\right), 2879\left(\mathrm{w}, \mathrm{v}\left[\mathrm{CH}_{3}\right]\right), 1713(\mathrm{~m}$, v[CO]), 1596, $1504\left(\mathrm{~m}, \mathrm{~m}, \mathrm{v}\left[\mathrm{C}-\mathrm{C}_{\mathrm{ar}}\right]\right), 1421\left(\mathrm{~m}, \delta_{\mathrm{as}}\left[\mathrm{CH}_{3}\right]\right), 1336\left(\mathrm{~m}, \delta_{\text {sy }}\left[\mathrm{CH}_{3}\right]\right), 1308(\mathrm{~m}$, $\mathrm{v}_{\mathrm{as}}\left[\mathrm{SO}_{2}\right], 1164\left(\mathrm{~s}, \mathrm{v}_{\mathrm{sy}}\left[\mathrm{SO}_{2}\right]\right), 843,822\left(\mathrm{~m}, \mathrm{~m}, \delta\left[\mathrm{CH}_{\mathrm{ar}}\right]\right) \mathrm{cm}^{-1}$. - MS (EI, I, $\left.70 \mathrm{eV}\right): \mathrm{m} / z(\%)=$ 278 (100) $\left[\mathrm{M}^{+}-\mathrm{C}_{2} \mathrm{H}_{3} \mathrm{O}\right], 155$ (47) $\left[\mathrm{C}_{7} \mathrm{H}_{7} \mathrm{SO}_{2}^{+}\right], 91$ (65) $\left[\mathrm{C}_{7} \mathrm{H}_{7}{ }^{+}\right], 77$ (1) $\left[\mathrm{C}_{6} \mathrm{H}_{5}^{+}\right] .-$HRMS (I): calcd.: $322.0913\left[\mathrm{M}^{+}+\mathrm{H}\right]$, found: $322.0911 .-\mathrm{C}_{16} \mathrm{H}_{16} \mathrm{FNO}_{3} \mathrm{~S}(321.37 \mathrm{~g} / \mathrm{mol})$, calcd.: $\mathrm{C} 59.80$, H 5.02, N 4.36, found: C 59.98, H 4.97, N 4.46.

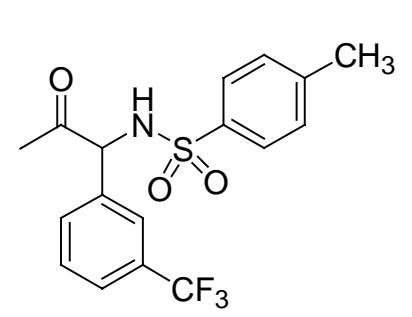

\section{2-(3'-Trifluormethylphenyl)-2-(4'"-toluene)sulfonylaminopropa-} none 6e: The compound was synthesized following GP 3, using 2(3'-trifluormethylphenyl)propanone $\quad(0.202 \quad \mathrm{~g}, \quad 1.00 \mathrm{mmol})$, chloramine-T (0.423 g, $1.50 \mathrm{mmol})$, and L-proline $(2.30 \mathrm{mg}$, $0.02 \mathrm{mmol})$ in acetonitrile $(5 \mathrm{~mL})$ within $2 \mathrm{~d}$. Flash chromatography on silica (diethylether/pentane 1:2) delivered $0.308 \mathrm{~g}(\mathrm{xxx} \mathrm{mmol}, \mathrm{xx} \%)$ of a colorless solid. For reaction conditions, see Table 3. $-\mathrm{mp}=92{ }^{\circ} \mathrm{C} .-\mathrm{R}_{f}=0.43$ (diethylether/pentane 1:2). ${ }^{1} \mathrm{H}$ NMR (400 MHz, DMSO): $\delta=2.19$ (s, $3 \mathrm{H}, \mathrm{CORCH}_{3}$ ), 2.37 (s, $3 \mathrm{H}, \mathrm{C}^{4}{ }_{\mathrm{Ts}} \mathrm{CH}_{3}$ ), 3.42 (bs, $1 \mathrm{H}, \mathrm{NH}), 3.95$ (s, $\left.1 \mathrm{H}, \mathrm{CHR}_{2} \mathrm{NHR}\right), 7.32\left(\mathrm{~s}, 1 \mathrm{H}, \mathrm{C}^{2} \mathrm{H}_{\mathrm{ar}}\right), 7.37$ (d, J=8.0 Hz, $\left.2 \mathrm{H}, \mathrm{C}^{3}{ }^{\prime \prime} \mathrm{H}_{\mathrm{Ts}}\right)$, $7.47-7.61\left(\mathrm{~m}, 3 \mathrm{H}, \mathrm{CH}_{\mathrm{ar}}\right), 7.75\left(\mathrm{~d}, J=8.3 \mathrm{~Hz}, 2 \mathrm{H}, \mathrm{C}^{2}{ }^{\prime \prime} \mathrm{H}_{\mathrm{Ts}}\right)$ ppm. $-{ }^{13} \mathrm{C} \mathrm{NMR}(100 \mathrm{MHz}$, DMSO): $\delta=20.8\left(+, \mathrm{C}^{4}{ }^{\prime}{ }_{\mathrm{Ts}} \underline{\mathrm{CH}}_{3}\right), 29.6\left(+, \mathrm{CORCH}_{3}\right), 48.6\left(+, \mathrm{CHR}_{3}\right), 124.3$ (q, q, $J=272.2$ $\left.\mathrm{Hz}, \mathrm{C}^{3}{ }^{\prime}{ }_{\mathrm{ar}} \underline{\mathrm{F}}_{3}\right), 123.1\left(+, \mathrm{q}, J=3.9 \mathrm{~Hz}, \mathrm{C}^{4}{ }^{\prime}{ }_{\mathrm{rr}} \mathrm{H}\right), 125.6\left(+, \mathrm{C}^{3{ }^{\prime \prime}}{ }_{\mathrm{Ts}} \mathrm{H}\right), 126.3(+, \mathrm{q}, J=3.8 \mathrm{~Hz}$, 


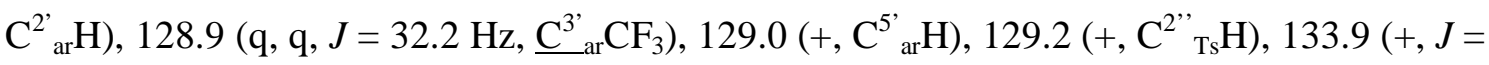
$\left.0.9 \mathrm{~Hz}, \mathrm{C}^{6}{ }_{\mathrm{ar}} \mathrm{H}\right), 136.3$ (q, $\underline{\mathrm{C}}^{1}{ }_{\mathrm{ar}} \mathrm{CHR}_{3}$ ), 141.4 (q, $\left.\mathrm{C}^{1,}{ }_{\mathrm{Ts}} \mathrm{SO}_{2}\right), 141.8\left(\mathrm{q}, \underline{\mathrm{C}}^{4}{ }_{\mathrm{Ts}} \mathrm{CH}_{3}\right), 205.3$ (q, $\left.\mathrm{CORCH}_{3}\right)$ ppm. - IR (KBr): v = $3268(\mathrm{~m}, \mathrm{v}[\mathrm{NH}]), 3070\left(\mathrm{w}, \mathrm{v}\left[\mathrm{CH}_{\mathrm{ar}}\right]\right), 2931\left(\mathrm{w}, \mathrm{v}\left[\mathrm{CH}_{3}\right]\right), 1731$ (m, v[CO]), 1599, 1496 (m, w, v[C-C $\left.\left.\mathrm{C}_{\mathrm{ar}}\right]\right), 1452\left(\mathrm{~m}, \delta_{\mathrm{as}}\left[\mathrm{CH}_{3}\right]\right), 1332\left(\mathrm{~m}, \delta_{\text {sy }}\left[\mathrm{CH}_{3}\right]\right), 1277(\mathrm{~m}$, $v_{\text {as }}\left[\mathrm{SO}_{2}\right], 1162\left(\mathrm{~m}, v_{\mathrm{sy}}\left[\mathrm{SO}_{2}\right]\right), 853,809\left(\mathrm{~m}, \mathrm{~m}, \delta\left[\mathrm{CH}_{\mathrm{ar}}\right]\right) \mathrm{cm}^{-1}$. $-\mathrm{MS}(\mathrm{EI}, \mathrm{I}, 70 \mathrm{eV}): \mathrm{m} / z(\%)=$ $328(54)\left[\mathrm{M}^{+}-\mathrm{C}_{2} \mathrm{H}_{3} \mathrm{O}\right], 173(100)\left[\mathrm{M}^{+}-\mathrm{C}_{7} \mathrm{H}_{7} \mathrm{SO}_{2}\right], 155$ (58) $\left[\mathrm{C}_{7} \mathrm{H}_{7} \mathrm{SO}_{2}{ }^{+}\right], 145$ (34) $\left[\mathrm{C}_{7} \mathrm{H}_{4} \mathrm{~F}_{3}{ }^{+}\right]$, 91 (94) $\left[\mathrm{C}_{7} \mathrm{H}_{7}^{+}\right], 77$ (5) $\left[\mathrm{C}_{6} \mathrm{H}_{5}^{+}\right]$. - HRMS (I): calcd.: 371.0803, found: 371.0804. $\mathrm{C}_{17} \mathrm{H}_{16} \mathrm{~F}_{3} \mathrm{NO}_{3} \mathrm{~S}$ (371.37 g/mol), calcd.: C 54.98, H 4.34, N 3.77, found: C 55.21, H 4.44, N 3.87 .

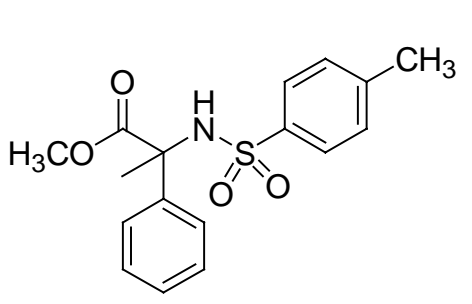

2-Phenyl-2-(4'-toluene)sulfonylaminopropionic acid methyl ester 7: 0.455 g $\quad(1.50$ mmol) 2-Phenyl-2-(4'toluene)sulfonylaminopropionaldehyde was dissolved in acetonitrile $(5 \mathrm{ml})$ along with $0.050 \mathrm{~g}(0.42 \mathrm{mmol}) \mathrm{NaH}_{2} \mathrm{PO}_{4}$ in $2 \mathrm{ml}$ water and $0.14 \mathrm{ml}(1.50 \mathrm{mmol}) 35 \% \mathrm{H}_{2} \mathrm{O}_{2}$. To the resulting mixture was added a solution of $\mathrm{NaClO}_{2}(0.226 \mathrm{~g}, 2.60 \mathrm{mmol})$ in $5 \mathrm{ml}$ of water dropwise, keeping the temperature of the mixture below $10{ }^{\circ} \mathrm{C}$. After the mixture was stirred for $12 \mathrm{~h}, \mathrm{Na}_{2} \mathrm{SO}_{3}(0.015 \mathrm{~g}, 0.12 \mathrm{mmol})$ was added and the resulting mixture was acidified ( $\mathrm{pH}$ $=2-3$ ) with $10 \%$ aqueous hydrochloric acid. The resulting mixture was partitioned between brine and dichloromethane, the layers were separated, and the aqueous layer was extracted with dichloromethane. The combined organic extracts were washed with brine, dried, and concentrated under reduced pressure to give a residue that was taken up in toluene/methanol 1: 2 and treated with an ethereal solution of trimethylsilyldiazomethane at $0{ }^{\circ} \mathrm{C}$ for 5 min until bubbling subsided. The excess trimethylsilyldiazomethane was quenched with a few drops of acetic acid and the solvent was removed in vacuo. Column chromatography on silica (diethylether/pentane, 1:2) delivered $0.460 \mathrm{~g}$ (1.38 mmol, $92 \%$ over 2 steps) of a colorless solid. $-\mathrm{mp}=109{ }^{\circ} \mathrm{C} .-\mathrm{R}_{f}=0.25$ (diethylether/pentane, 1:2). $-{ }^{1} \mathrm{H}$ NMR (400 MHz, $\left.\mathrm{CDCl}_{3}\right)$ : $\delta=1.84\left(\mathrm{~s}, 3 \mathrm{H}, \mathrm{CR}_{3} \mathrm{CH}_{3}\right), 2.30\left(\mathrm{~s}, 3 \mathrm{H}, \mathrm{C}^{4}{ }_{\mathrm{Ts}} \mathrm{CH}_{3}\right), 3.58\left(\mathrm{~s}, 3 \mathrm{H}, \mathrm{CO}_{2} \mathrm{CH}_{3}\right), 5.88(\mathrm{bs}, 1 \mathrm{H}, \mathrm{NH})$, $7.06\left(\mathrm{~d}, J=8.0 \mathrm{~Hz}, 2 \mathrm{H}, \mathrm{C}^{3} \mathrm{H}_{\mathrm{Ts}}\right), 7.09-7.17\left(\mathrm{~m}, 5 \mathrm{H}, \mathrm{CH}_{\mathrm{Ph}}\right), 7.42\left(\mathrm{~d}, J=8.3 \mathrm{~Hz}, 2 \mathrm{H}, \mathrm{C}^{2} \mathrm{H}_{\mathrm{Ts}}\right)$ ppm. $-{ }^{13} \mathrm{C}$ NMR $\left(100 \mathrm{MHz}, \mathrm{CDCl}_{3}\right): \delta=21.5\left(+, \mathrm{C}^{4}{ }_{\mathrm{Ts}} \underline{\mathrm{CH}_{3}}\right), 23.7\left(+, \mathrm{CR}_{3} \underline{\mathrm{C}}_{3}\right), 53.3(+$, $\left.\mathrm{CO}_{2} \mathrm{CH}_{3}\right), 64.4\left(\mathrm{q}, \mathrm{CR}_{4}\right), 125.9\left(\mathrm{C}_{\mathrm{Ph}} \mathrm{H}\right), 126.9\left(+, \mathrm{C}^{3}{ }_{\mathrm{TS}} \mathrm{H}\right), 128.1\left(+, \mathrm{C}^{4}{ }_{\mathrm{Ph}} \mathrm{H}\right), 128.5\left(+, \mathrm{C}_{\mathrm{Ph}} \mathrm{H}\right)$,

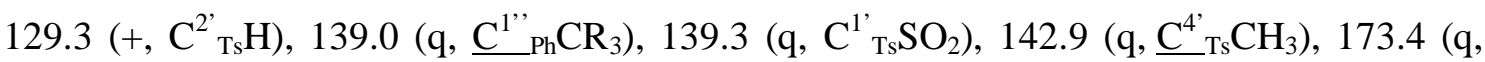
$\left.\mathrm{CO}_{2} \mathrm{CH}_{3}\right)$ ppm. - IR (KBr): v = $3257(\mathrm{~m}, \mathrm{v}[\mathrm{NH}]), 3068,3035\left(\mathrm{w}, \mathrm{w}, \mathrm{v}\left[\mathrm{CH}_{\mathrm{ar}}\right]\right), 2956,2928$ (w, 
$\left.\mathrm{w}, v\left[\mathrm{CH}_{3}\right]\right), 1745(\mathrm{~m}, \delta[\mathrm{NH}]), 1743(\mathrm{~m}, \mathrm{v}[\mathrm{CO}]), 1599,1494\left(\mathrm{w}, \mathrm{m}, \mathrm{v}\left[\mathrm{C}-\mathrm{C}_{\mathrm{ar}}\right]\right), 1446(\mathrm{~m}$, $\left.\delta_{\text {as }}\left[\mathrm{CH}_{3}\right]\right), 1371\left(\mathrm{~m}, \delta_{\text {sy }}\left[\mathrm{CH}_{3}\right]\right), 1328\left(\mathrm{~m}, v_{\text {as }}\left[\mathrm{SO}_{2}\right], 1168\left(\mathrm{~m}, \mathrm{v}_{\text {sy }}\left[\mathrm{SO}_{2}\right]\right), 855,810,765,737(\mathrm{~m}\right.$, $\left.\mathrm{m}, \mathrm{m}, \mathrm{w}, \delta\left[\mathrm{CH}_{\mathrm{ar}}\right]\right) \mathrm{cm}^{-1}$. - MS (EI, I, $\left.70 \mathrm{eV}\right): \mathrm{m} / z(\%)=274(100)\left[\mathrm{M}^{+}-\mathrm{C}_{2} \mathrm{H}_{3} \mathrm{O}_{2}\right], 155$ (17) $\left[\mathrm{C}_{7} \mathrm{H}_{7} \mathrm{SO}_{2}^{+}\right], 104$ (7) $\left[\mathrm{C}_{8} \mathrm{H}_{8}^{+}\right], 91$ (41) $\left[\mathrm{C}_{7} \mathrm{H}_{7}{ }^{+}\right], 77$ (5) $\left[\mathrm{C}_{6} \mathrm{H}_{5}^{+}\right] .-\mathrm{HRMS}(\mathrm{I})$ : calcd.: 333.1035, found: 333.1037. $-\mathrm{C}_{17} \mathrm{H}_{19} \mathrm{NO}_{4} \mathrm{~S}(333.40 \mathrm{~g} / \mathrm{mol})$, calcd.: C 61.24, H 5.74, N 4.20, found: C 61.37, H 5.89, N 4.28.

$\alpha$-Methylphenylglycine $\mathbf{8}^{[3,4]} \mathrm{A}$ heavy-walled borosilicate pressure bottle was
charged with $0.333 \mathrm{~g}(1.00 \mathrm{mmol})$ of 2-phenyl-2-(4'-toluene)sulfonyl-
aminopropionic acid methyl ester, phenol $(0.290,3.08 \mathrm{mmol})$ phenol and was sealed with a bushing, having a teflon-lined cap, before being immersed completely in an oil bath. The bath was maintained at $75{ }^{\circ} \mathrm{C}$ for $10-12 \mathrm{~h}$. The resulting solution was then concentrated in vacuo to about $10 \mathrm{ml}$. The crude solution was purified by ion-exchange chromatography (Amberlite IR-120 resin, $20 \mathrm{~g}$ ), eluting with $30 \mathrm{ml}$ water to remove impurities, then with $30 \mathrm{ml}$ of $10 \%$ ammonium hydroxide, followed by $30 \mathrm{ml}$ of conc. Ammonium hydroxide. Collection of the ammonium hydroxide eluate gave a solution of the ammonium salt of $\alpha$-methylphenylglycine which upon lyophilization yielded a colorless solid. Recrystallization from methanol/ether delivered $0.145 \mathrm{~g}(0.88 \mathrm{mmol}, 88 \%)$ as a colorless solid. NMR was in accordance with the literature. ${ }^{1} \mathrm{H}$ NMR (400 MHz, MeOD): $\delta=$ 1.90 (s, $3 \mathrm{H}, \mathrm{CR}_{3} \mathrm{CH}_{3}$ ), 4.92 (bs, $\left.\mathrm{NH}_{2} / \mathrm{CO}_{2} \mathrm{H}\right), 7.36-7.45$ (m, $\left.3 \mathrm{H}, \mathrm{CH}_{\mathrm{ar}}\right), 7.59-7.61$ (m, $2 \mathrm{H}$, $\left.\mathrm{CH}_{\mathrm{ar}}\right)$ ppm. $-{ }^{13} \mathrm{C} \mathrm{NMR}(100 \mathrm{MHz}, \mathrm{MeOD}): \delta=22.8\left(\mathrm{CR}_{3} \underline{\mathrm{CH}}_{3}\right), 64.1\left(\mathrm{CR}_{3} \mathrm{CH}_{3}\right), 127.1$

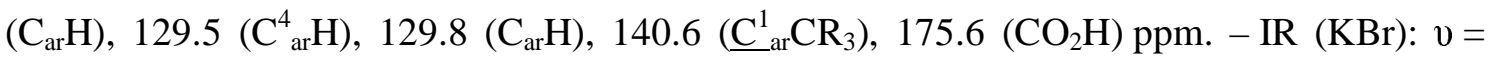
$3054(\mathrm{~m}, \mathrm{v}[\mathrm{OH}]), 2557(\mathrm{w}), 2135(\mathrm{w}), 1753(\mathrm{vw}), 1624\left(\mathrm{~m}, \delta\left[\mathrm{NH}_{3}{ }^{+}\right]\right), 1592\left(\mathrm{~m}, \mathrm{v}\left[\mathrm{COO}^{-}\right]\right.$, $1501\left(\mathrm{~m}, \delta\left[\mathrm{NH}_{3}^{+}\right]\right), 1446\left(\mathrm{~m}, \delta_{\mathrm{as}}\left[\mathrm{CH}_{3}\right], 1393\left(\mathrm{~m}, \mathrm{v}\left[\mathrm{COO}^{-}\right]\right), 1357\left(\mathrm{~m}, \mathrm{v}\left[\mathrm{CNH}_{2}\right], 876(\mathrm{~m}), 766\right.\right.$, $742\left(\mathrm{w}, \mathrm{m}, \delta\left[\mathrm{CH}_{\mathrm{ar}}\right]\right) \mathrm{cm}^{-1}$. - MS (EI): $\mathrm{m} / z(\%): 150$ (1) [ $\left.\mathrm{M}^{+}-\mathrm{NH}_{2}\right], 120$ (100) $\left[\mathrm{M}^{+}-\mathrm{CO}_{2} \mathrm{H}\right], 104$ (7) $\left[\mathrm{C}_{8} \mathrm{H}_{8}{ }^{+}\right], 91$ (2) $\left[\mathrm{C}_{7} \mathrm{H}_{7}{ }^{+}\right], 77(8)\left(\mathrm{C}_{6} \mathrm{H}_{5}{ }^{+}\right]$; - HRMS (EI): calcd. $120.0813\left[\mathrm{M}^{+}-\mathrm{CO}_{2} \mathrm{H}\right]$, found 120.0816 . 

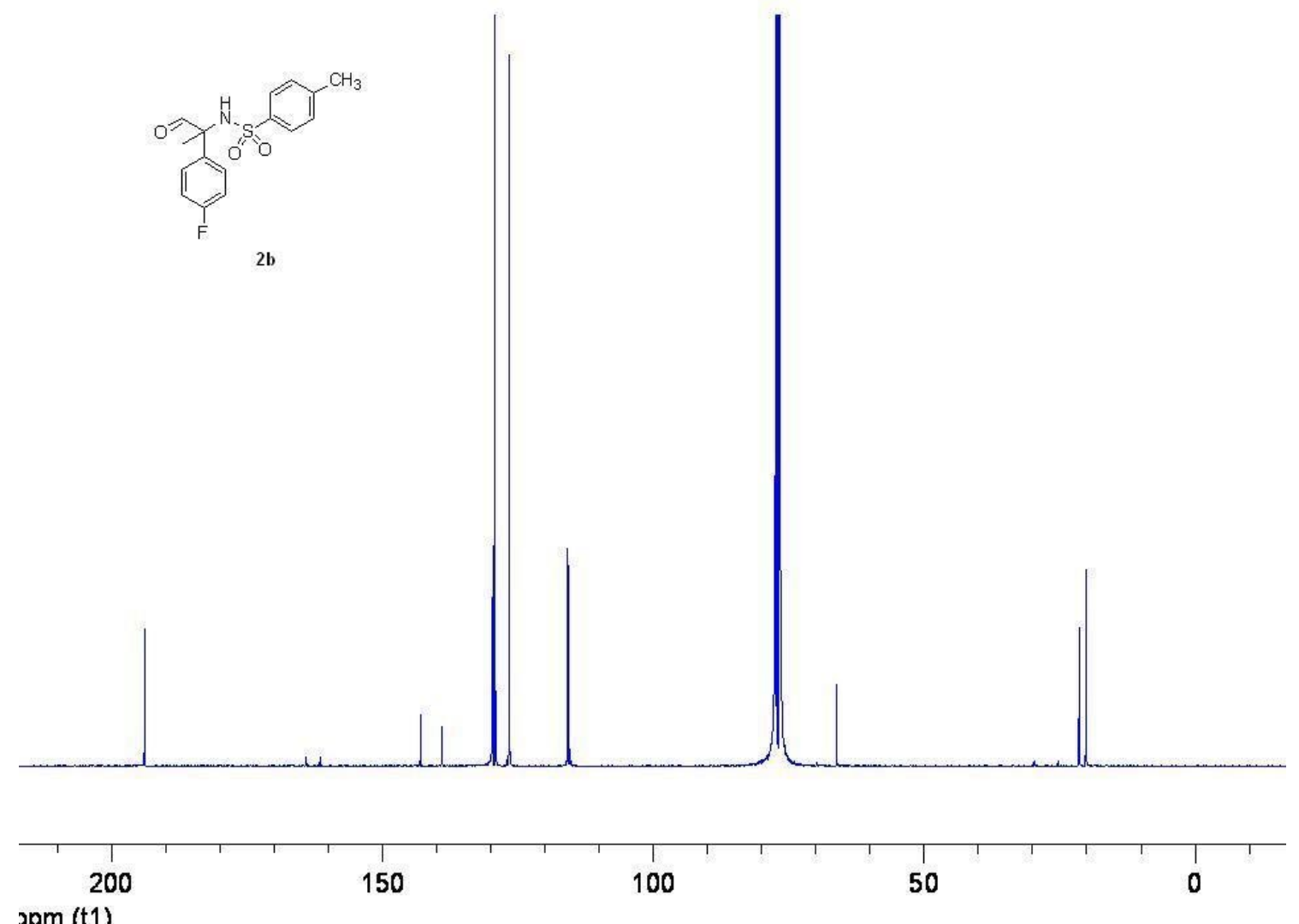

spm (t1)

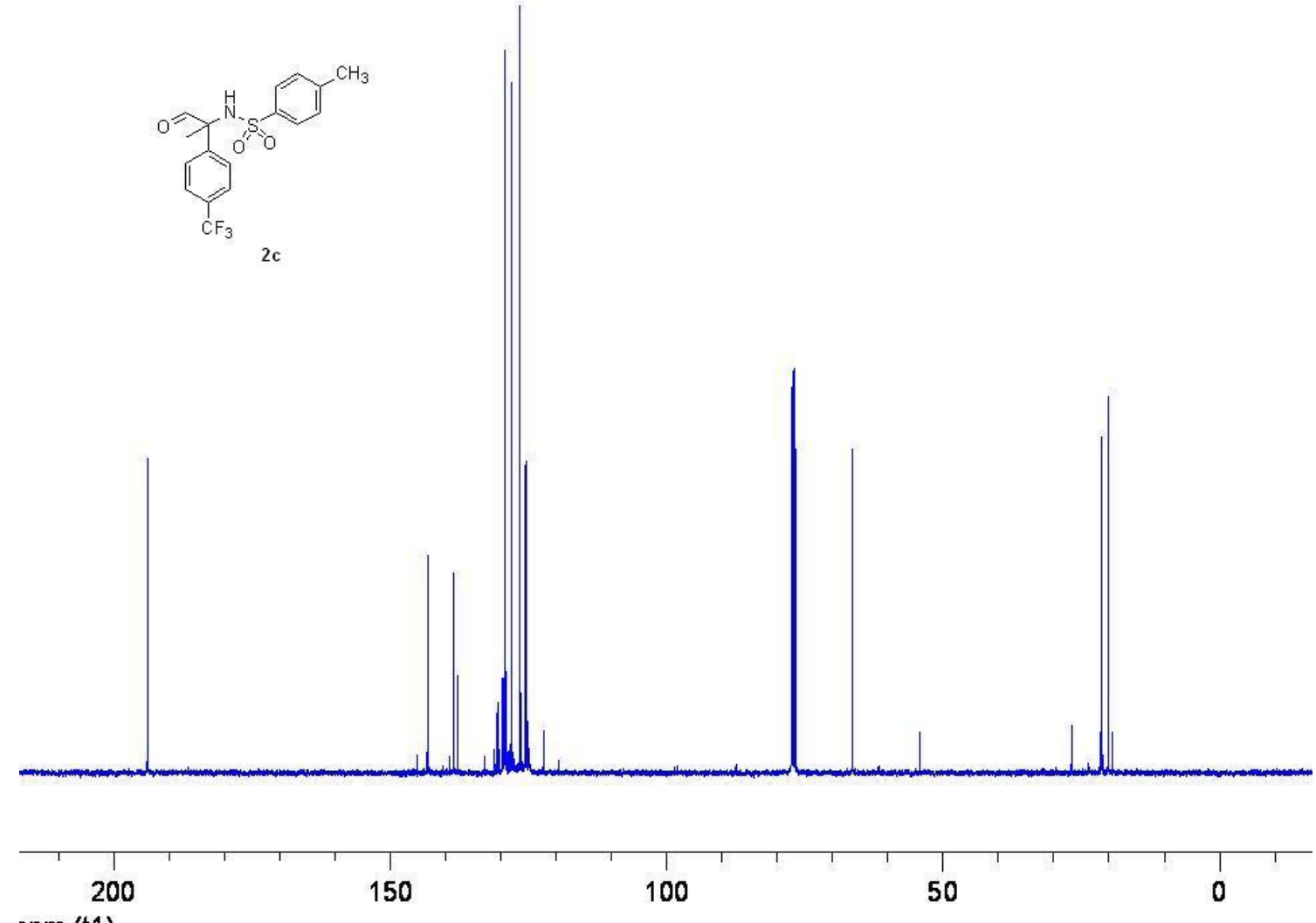

spm (t1) 

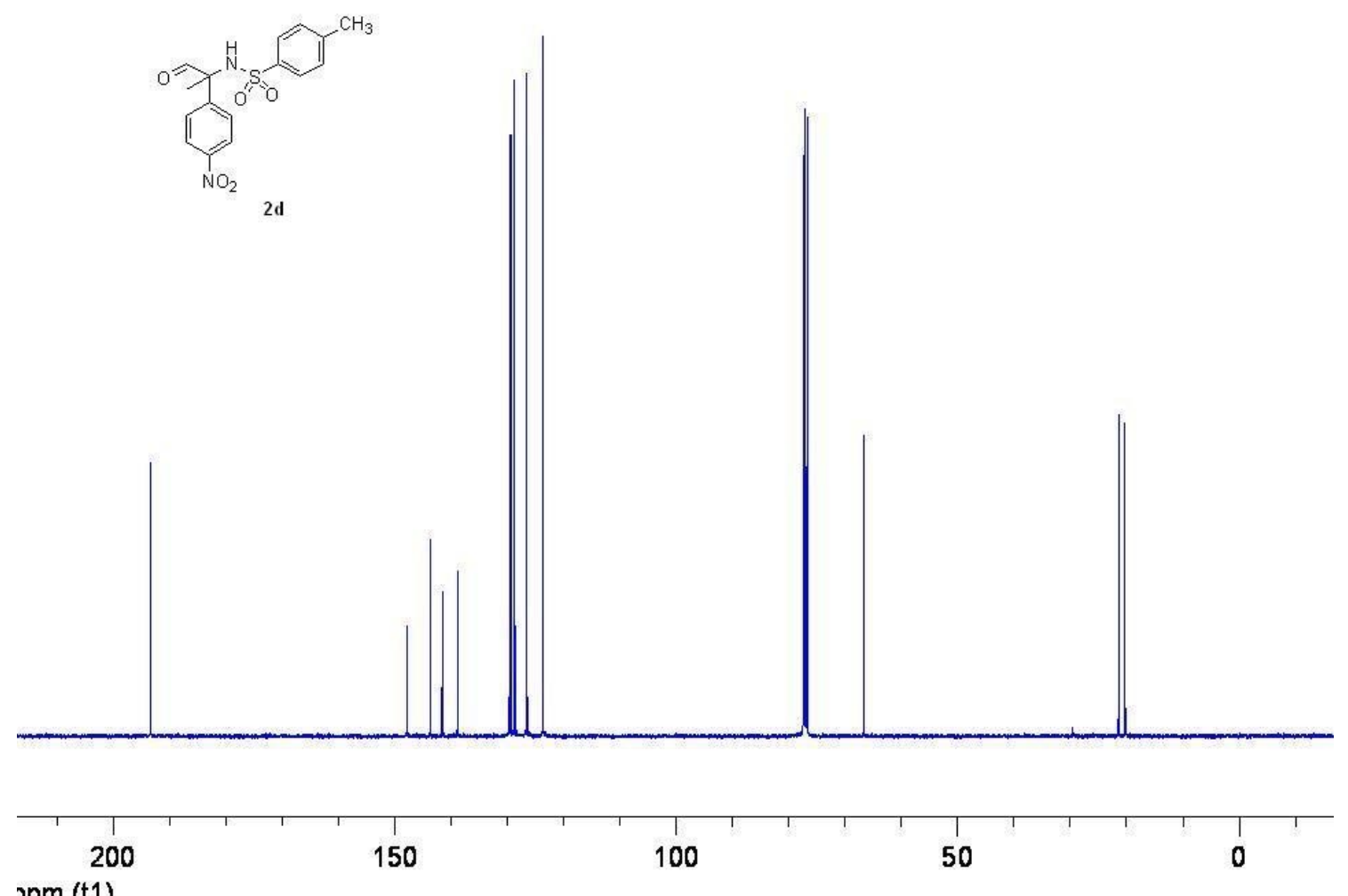
spm (t1)
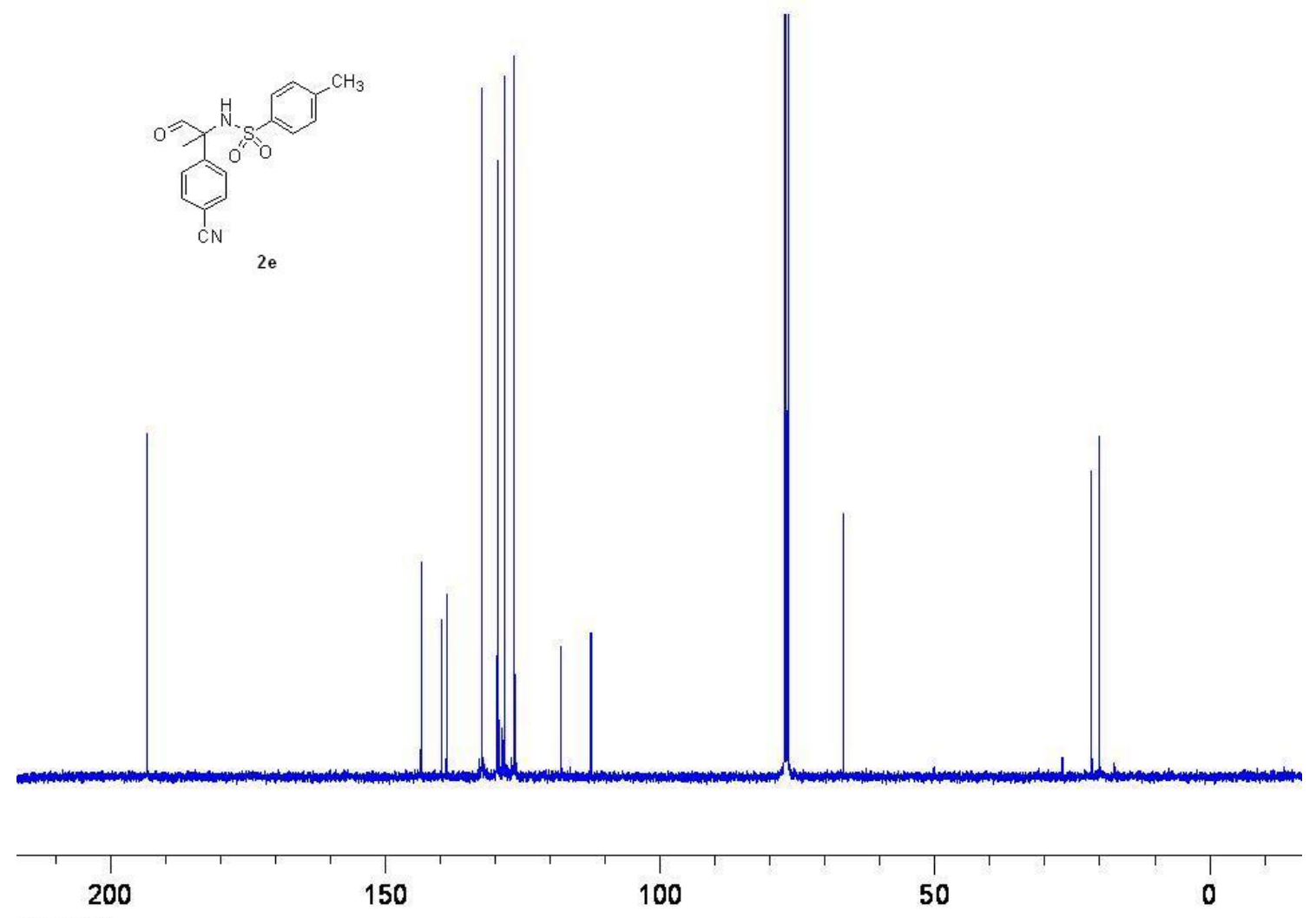

spm (t1) 

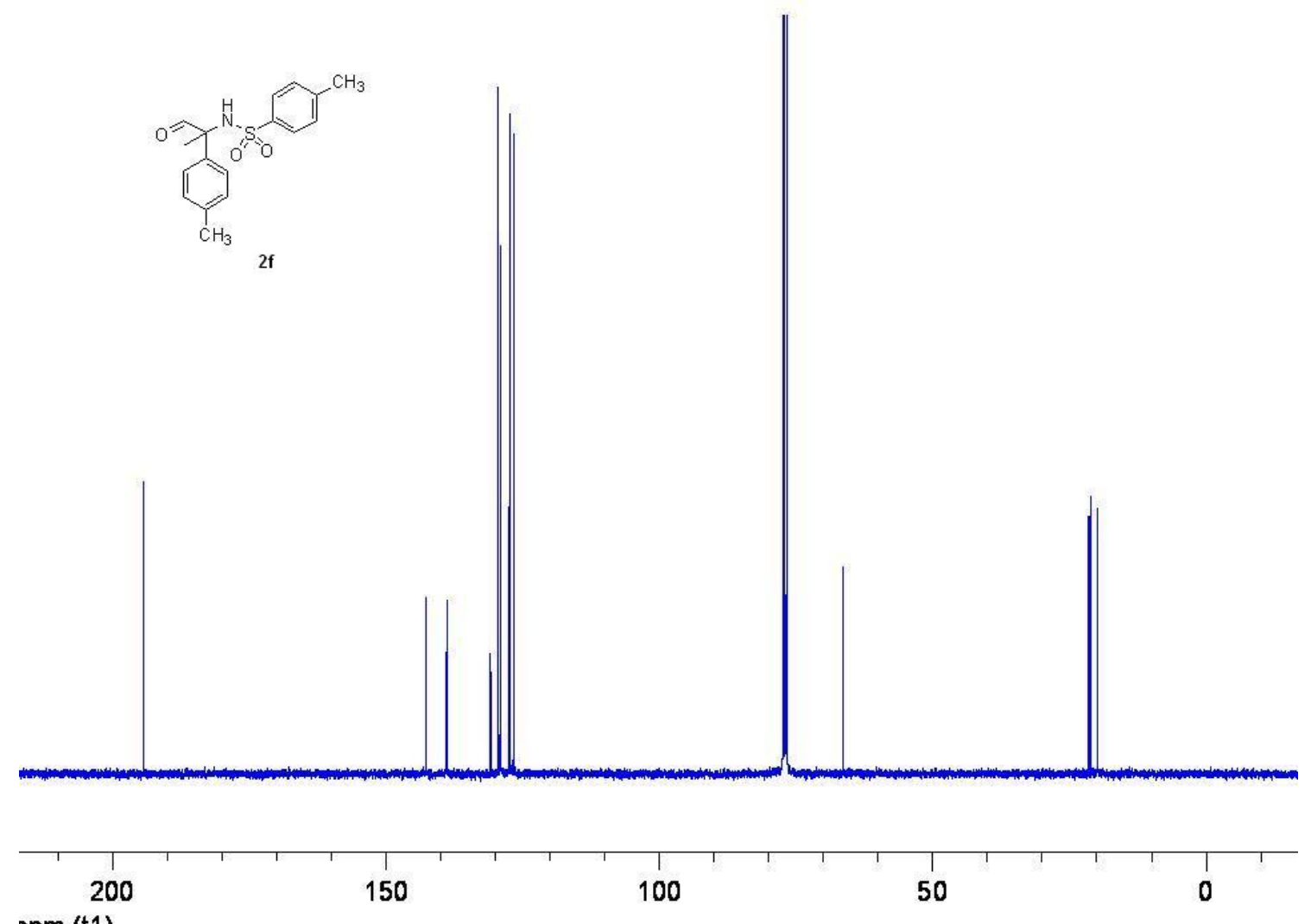

spm (t1)

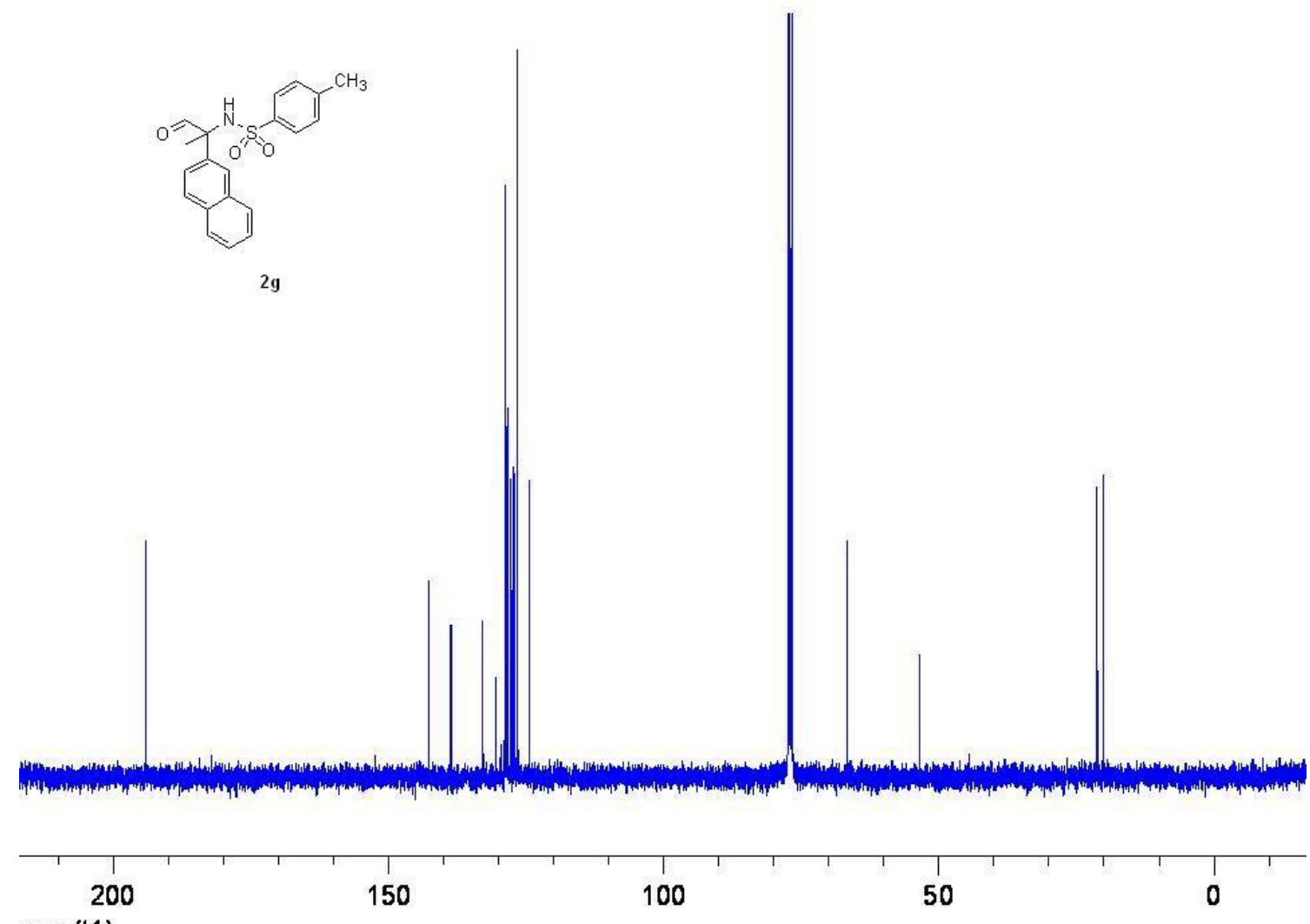

spm (t1) 

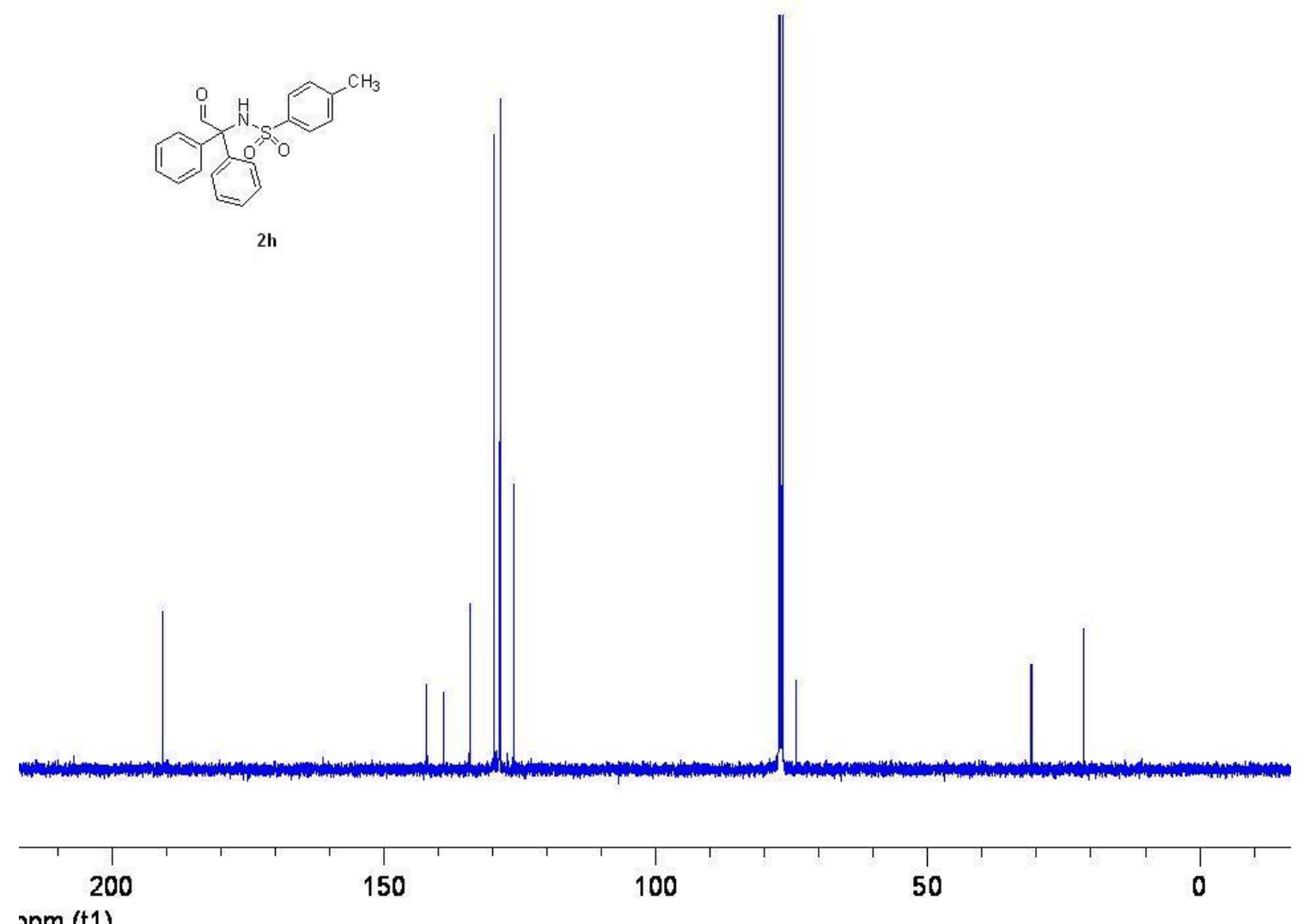

spm (t1)
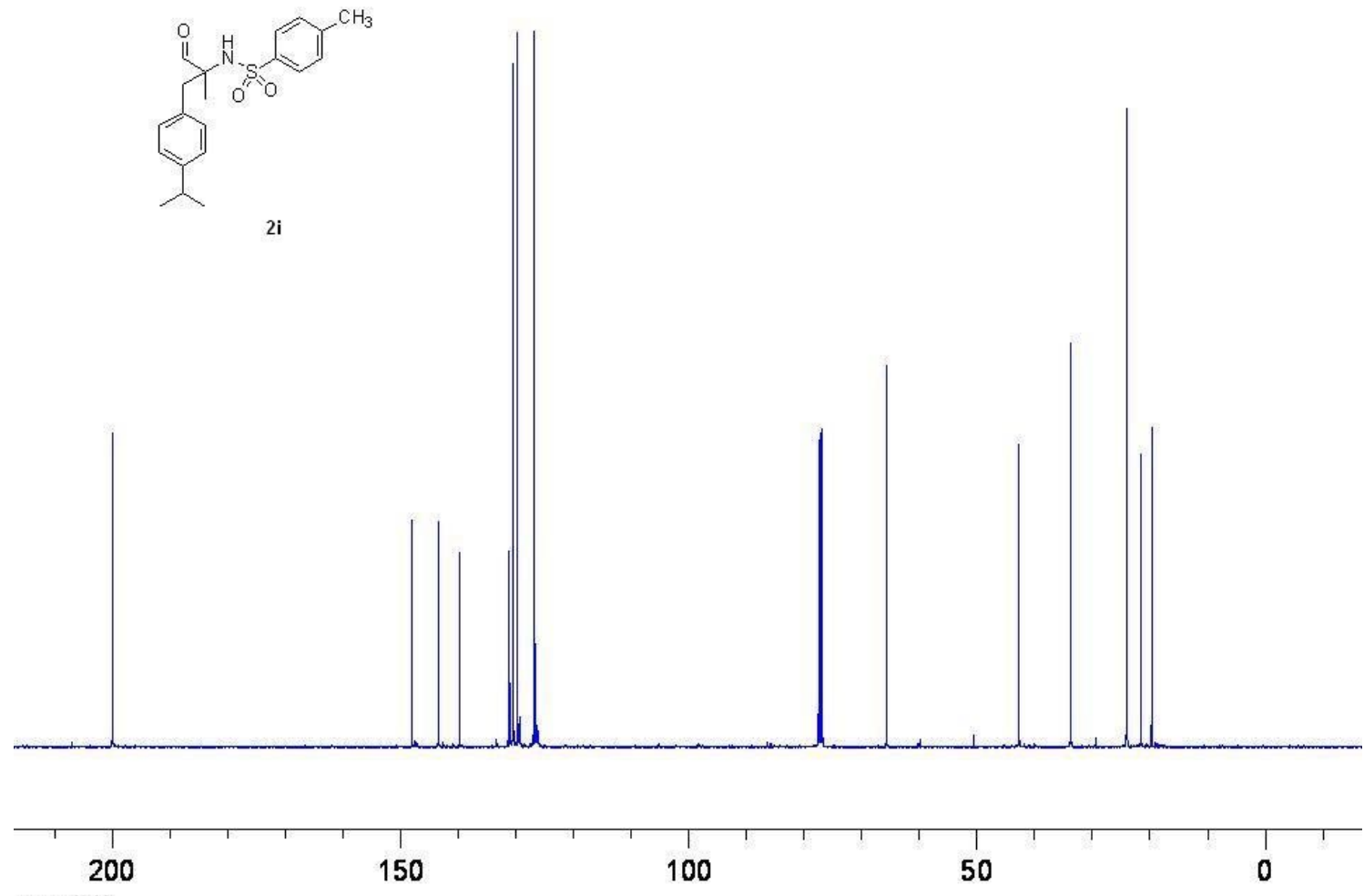

spm (t1) 

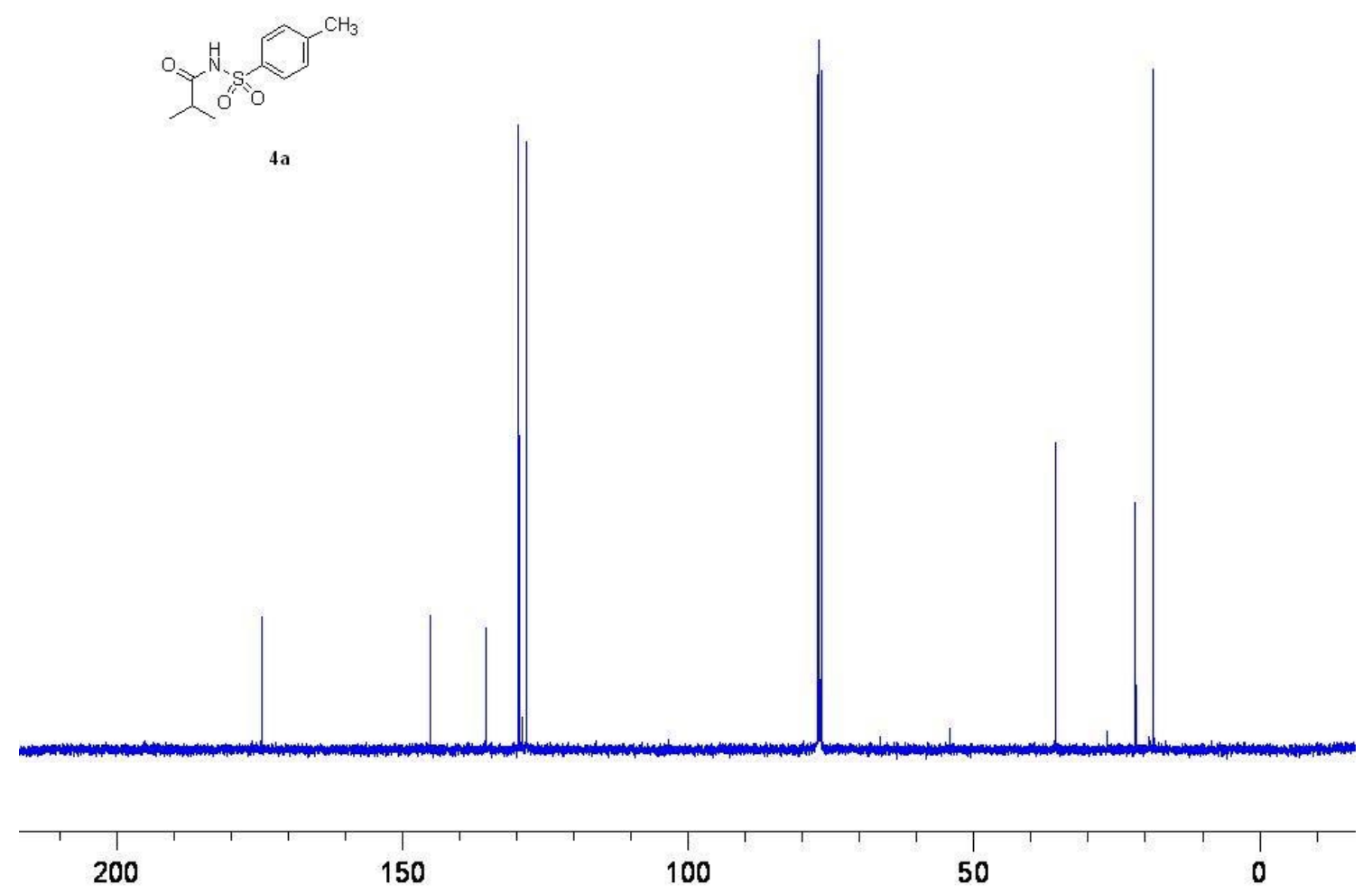
spm (t1)
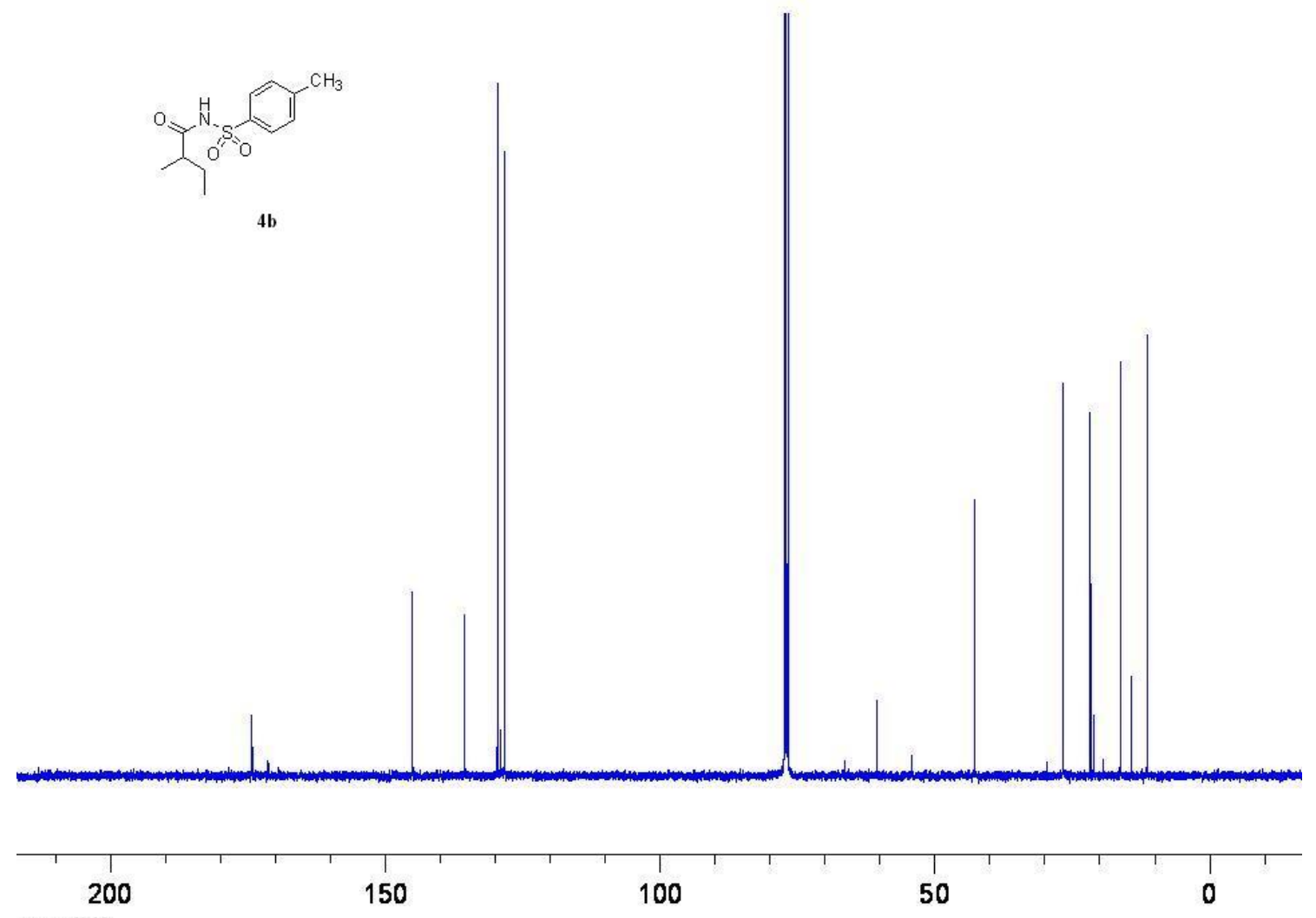

spm (t1) 

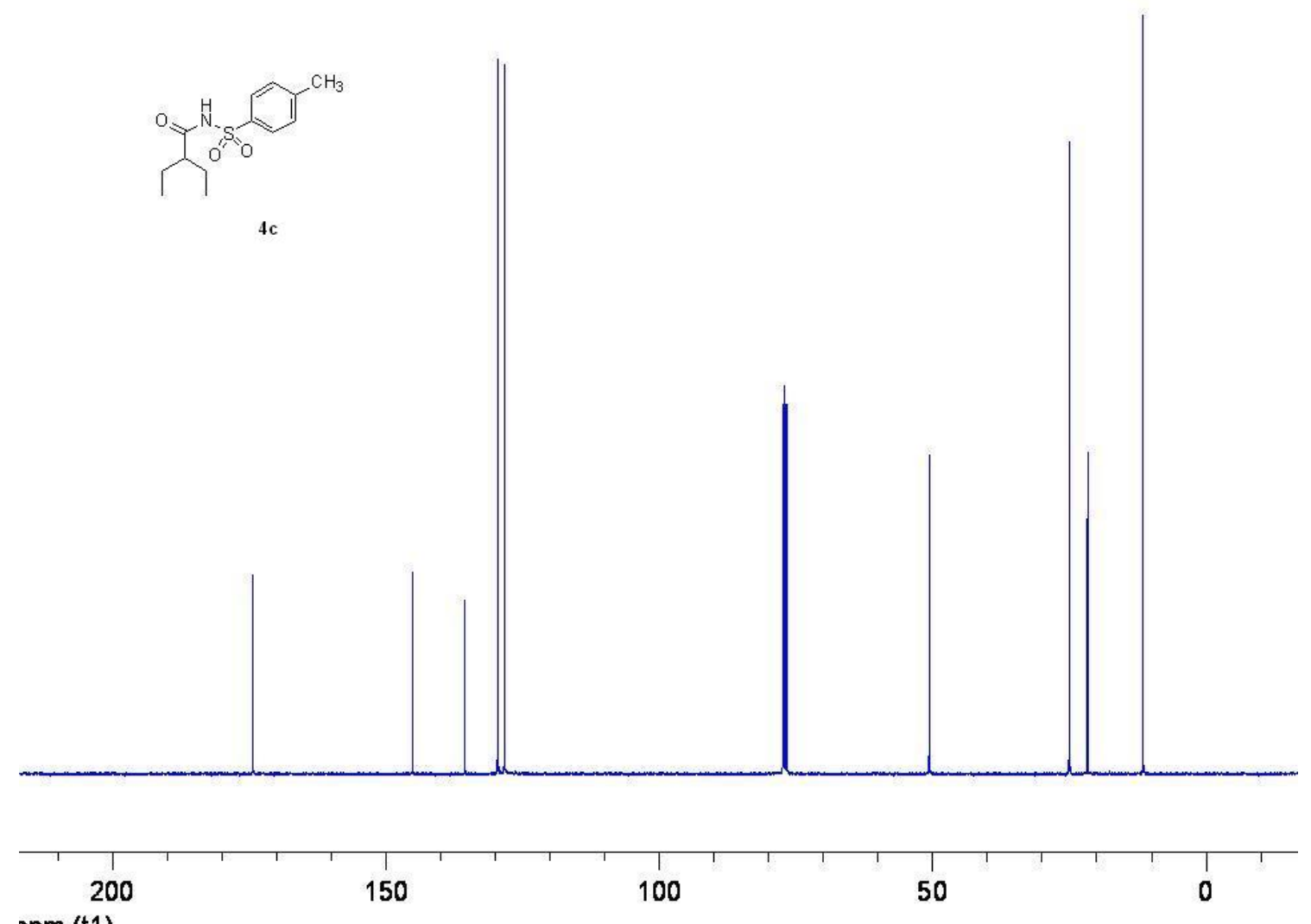
spm (t1)
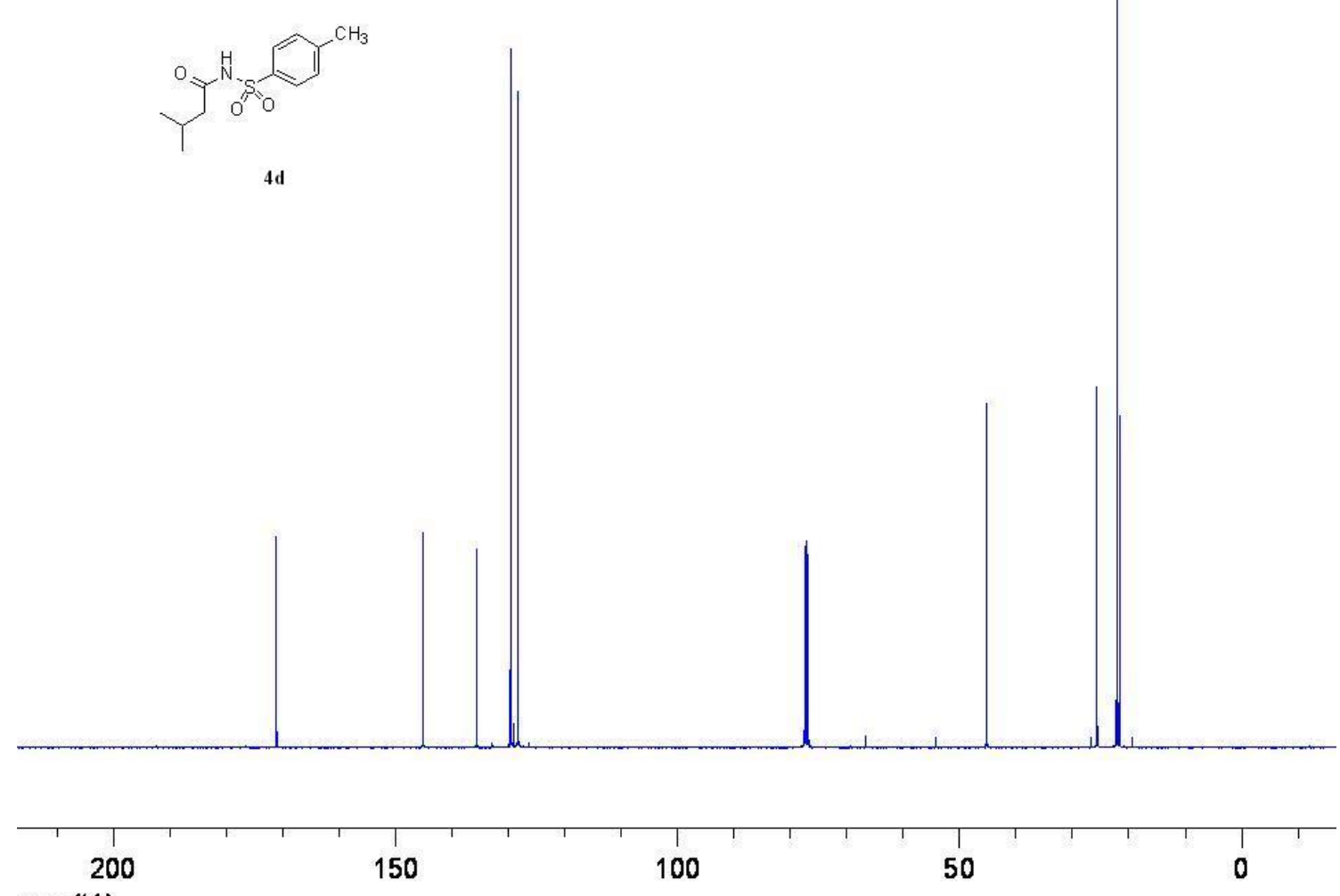

spm (t1) 

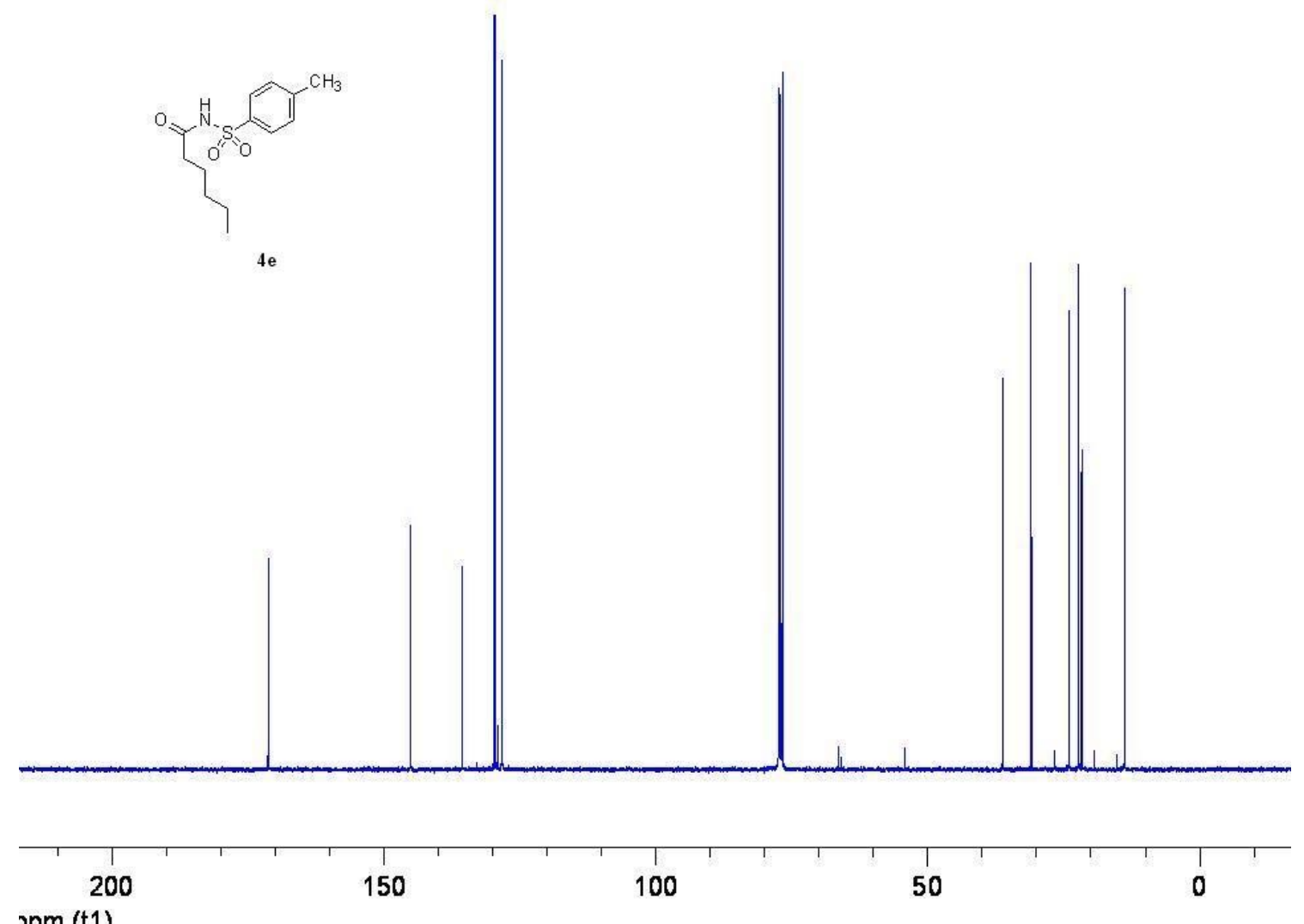
spm (t1)
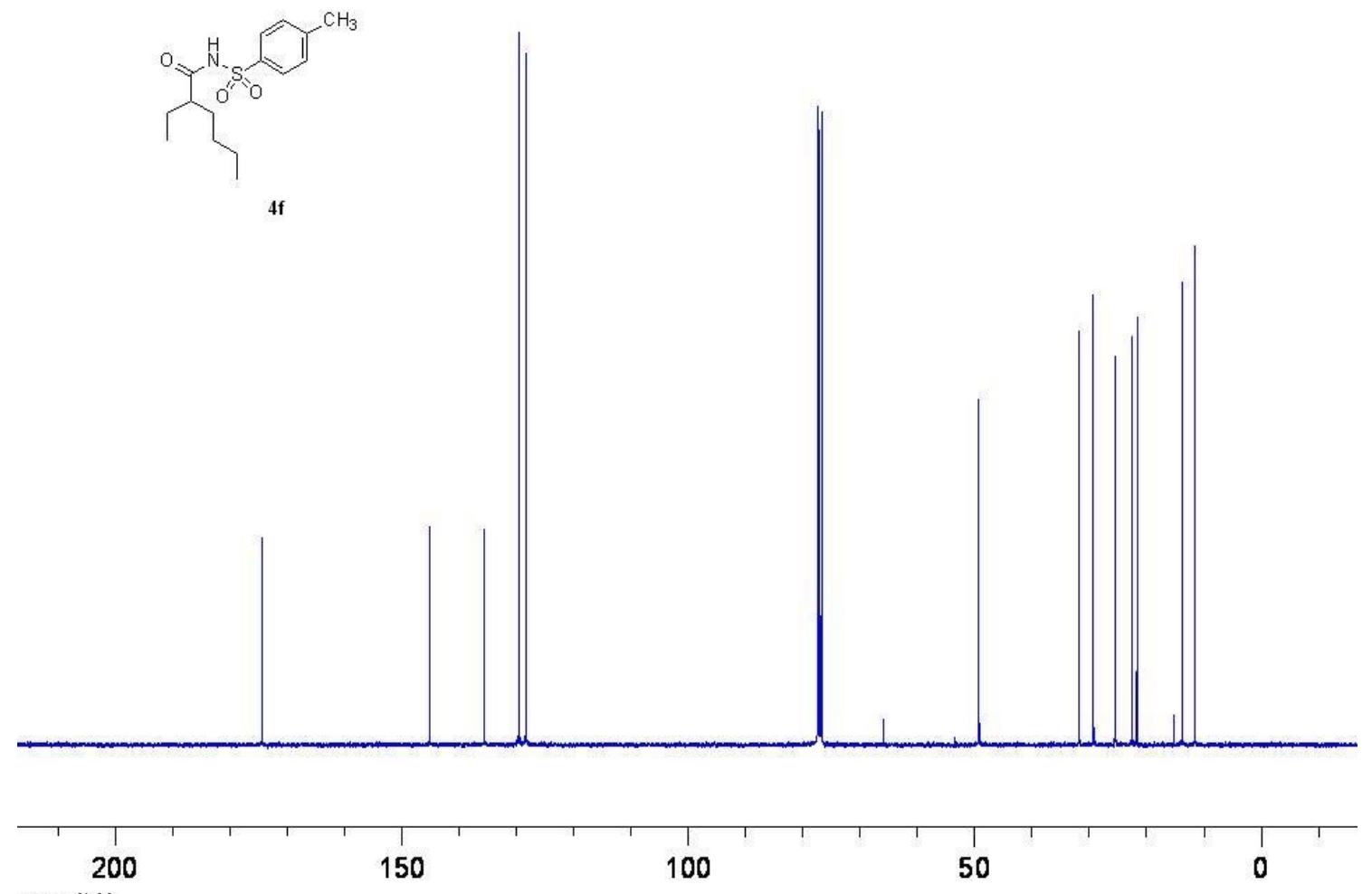

spm (t1) 

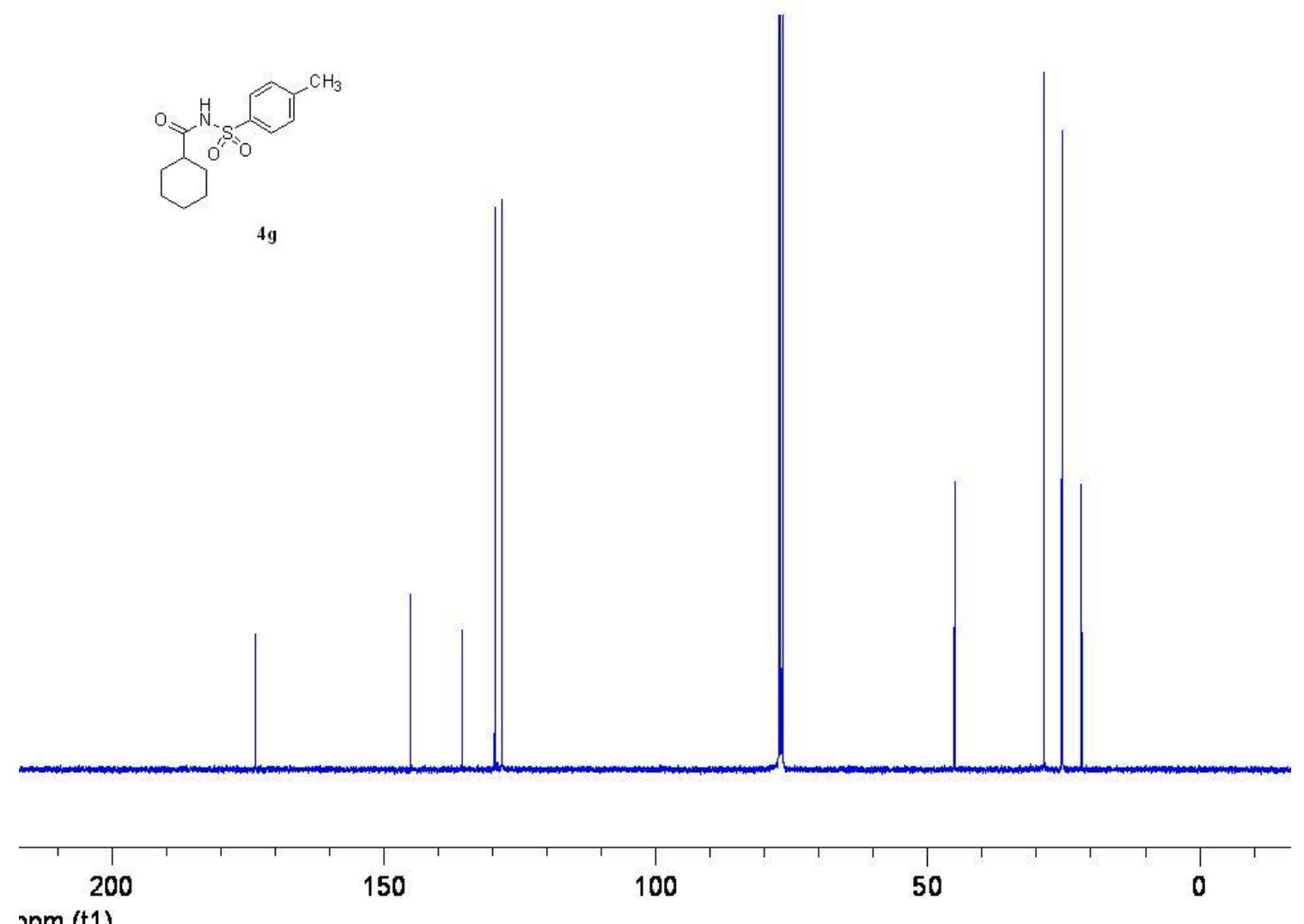
spm (t1)

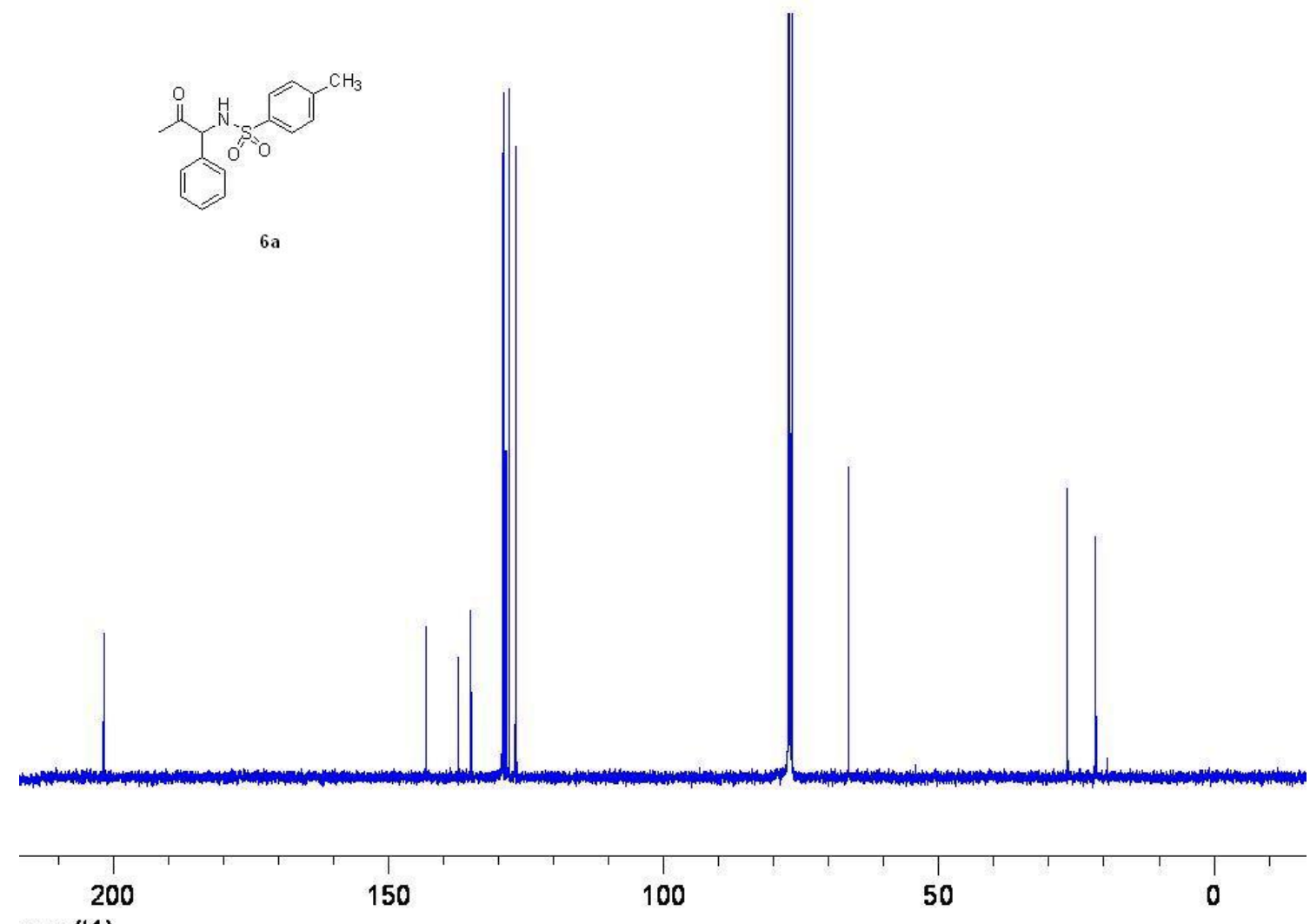

spm (t1) 

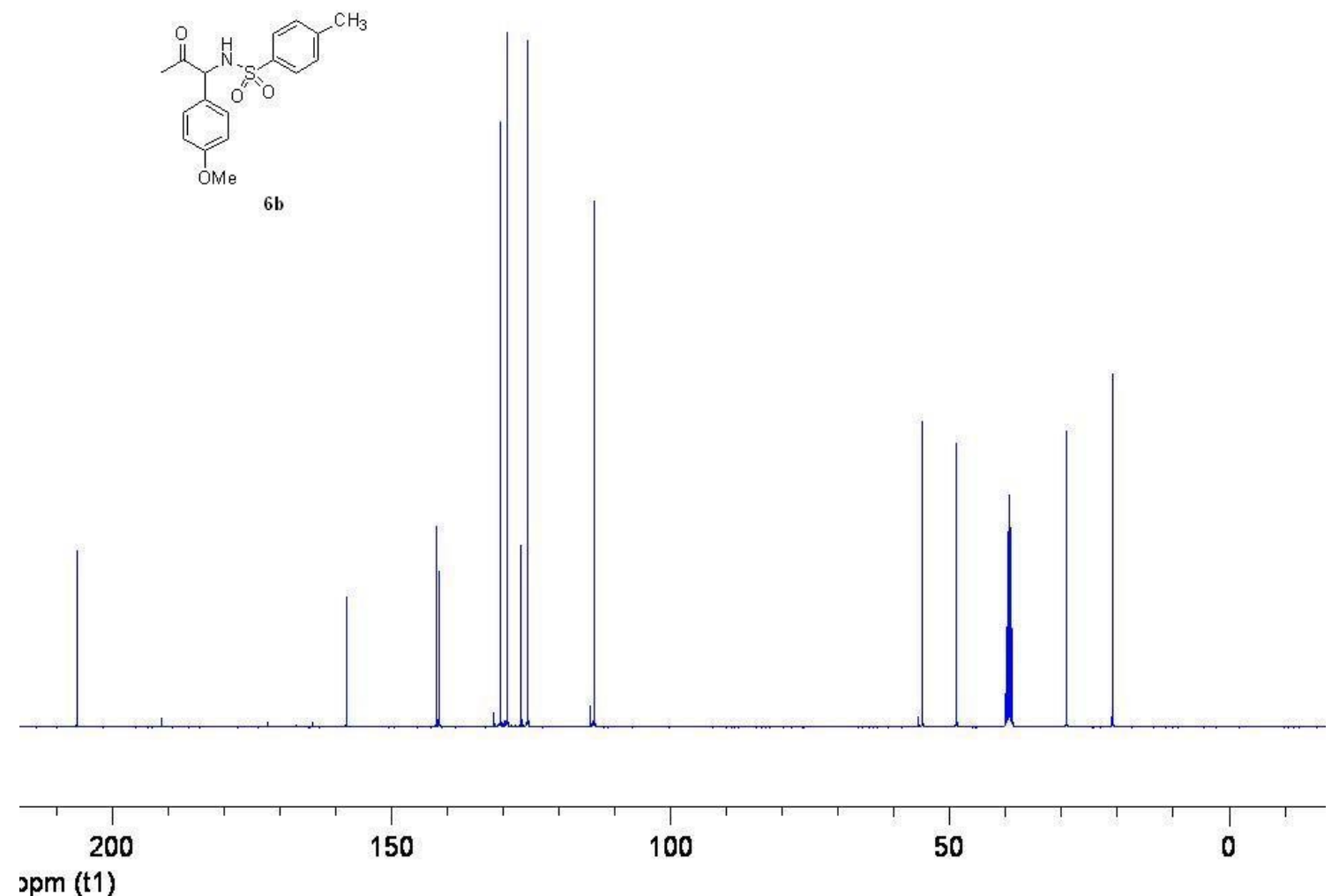

spm (t1)

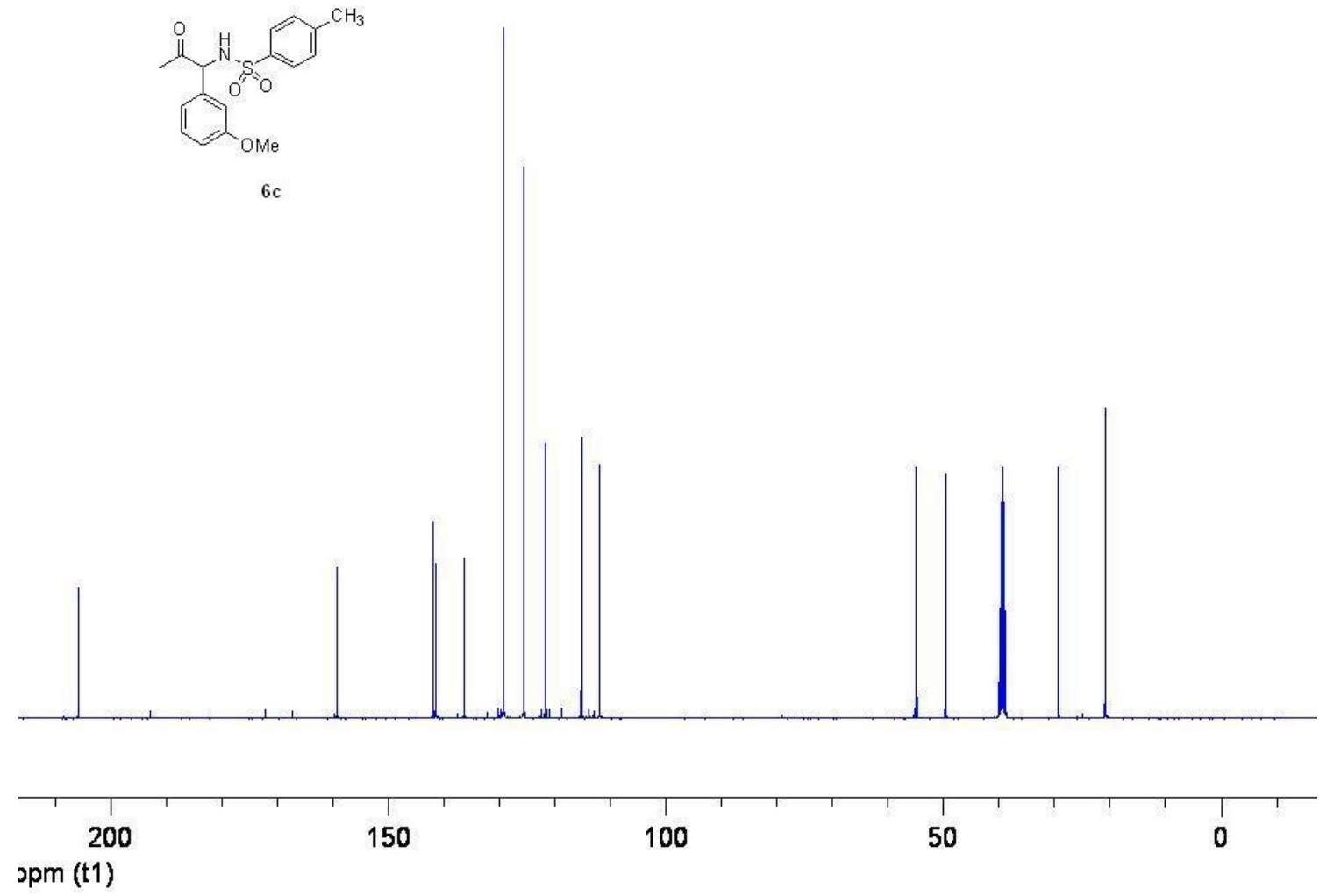



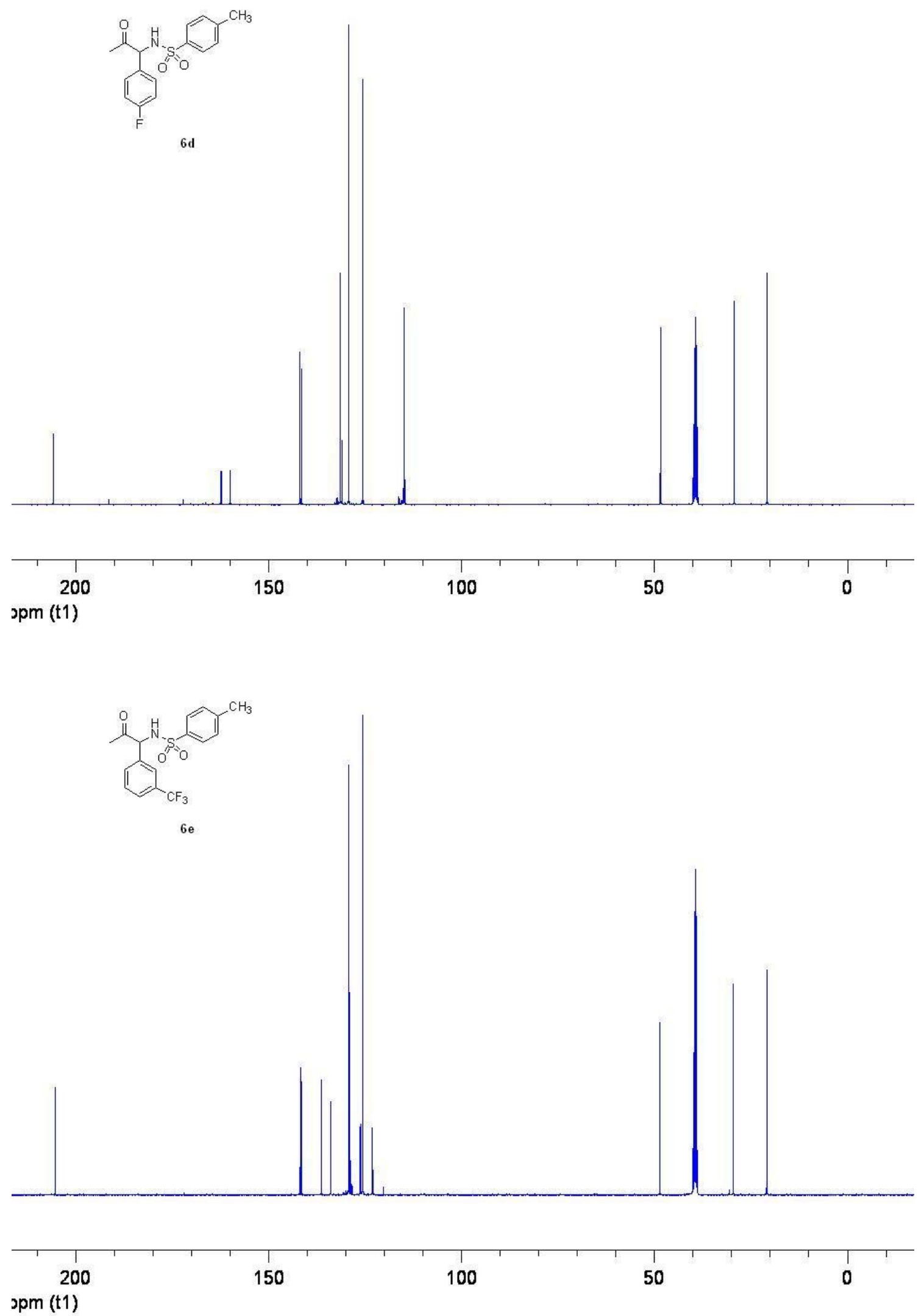

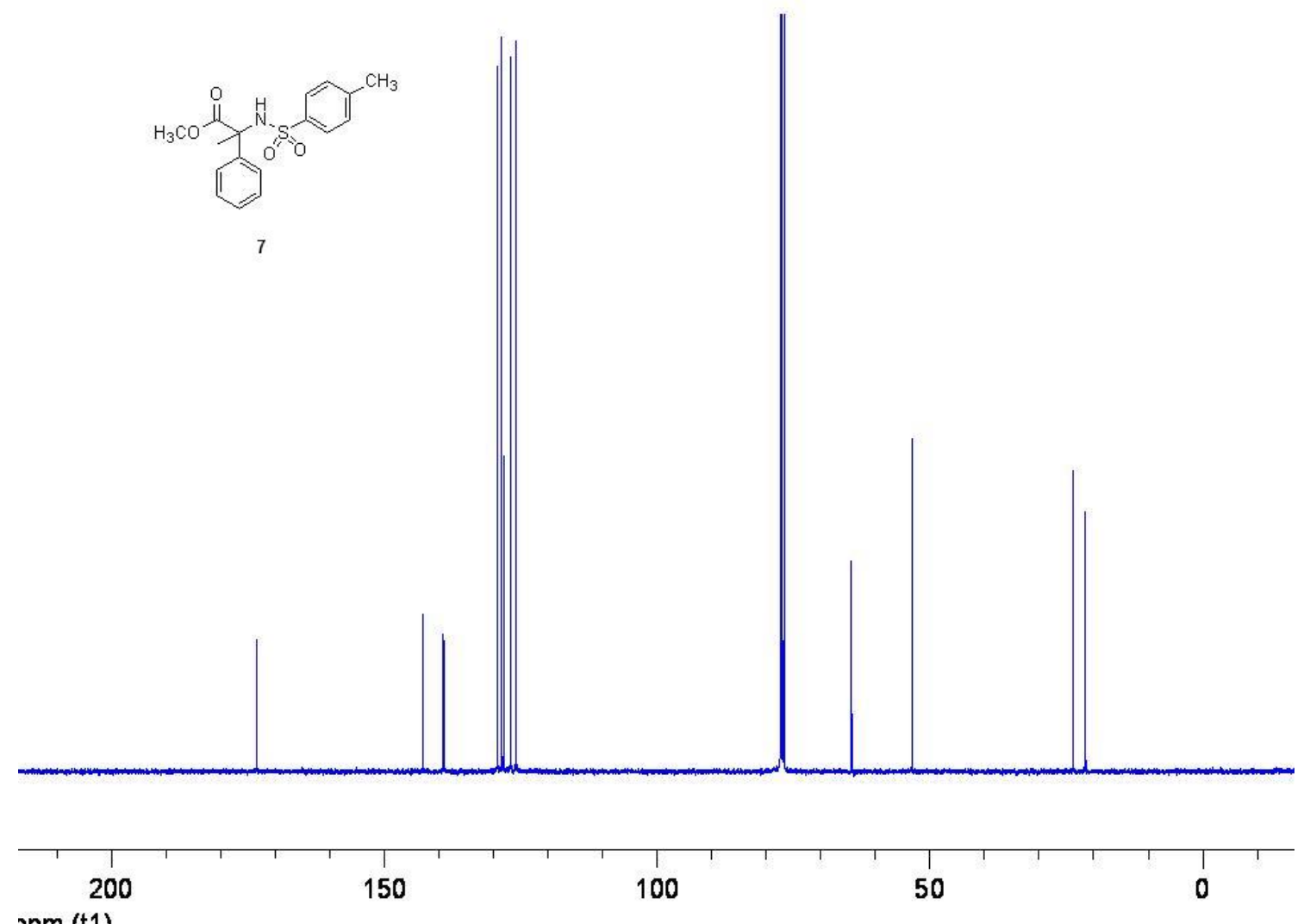
spm (t1)

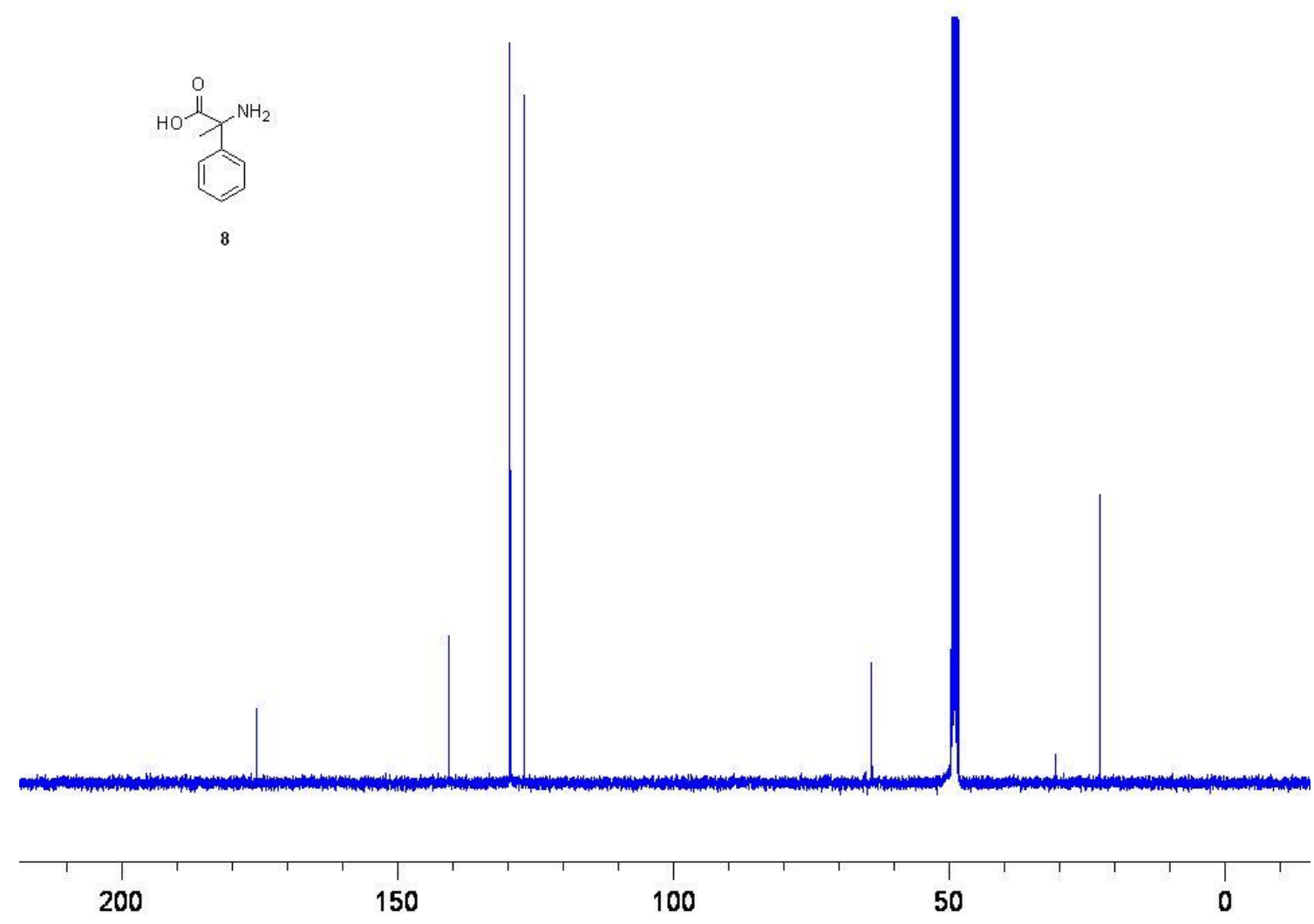

spm (t1) 
${ }^{[1]}$ H. E. Gottlieb, V. Kotlyar, A. Nudelman J. Org. Chem. 1997, 62, 7512.

${ }^{[2]}$ W. C. Still, M. Kahn, A. Mitra J. Org. Chem. 1978, 43, 2923.

${ }^{[3]}$ M.-X. Wang, S.-J. Lin, J. Liu, Q.-Y. Zheng Adv. Synth. Catal. 2004, 346, 439.

${ }^{[4]}$ N. Nagashima (Kaneka Corp., Japan) PCT Int. Appl. 2002, WO 2002022549. 